Physica Scripta., Vol 48, 297-325, 1993

ISSN (print): 1402-4896/48/3/008

doi; $10.1088 / 0031-8949 / 48 / 3 / 008$

(c) The Royal Swedish Academy of Sciences

This is an un-copyedited version of an article accepted for publication in Phys. Scr. IOP Publishing Ltd is not responsible for any errors or omissions in this version of the manuscript or any version derived from it. The definitive publisher authenticated version is available online at doi; $10.1088 / 0031-8949 / 48 / 3 / 008$

http://journals.iop.org/.

http://www.iop.org/EJ/journal/PhysScr

\title{
Transition Probabilities for Dipole Allowed Fine Structure Transitions in Si- like Ions: Si I, S III, Ar V and Ca VII
}

\author{
Sultana N. Nahar \\ Department of Astronomy, The Ohio State University, Columbus, OH 43210, U.S.A.
}

\begin{abstract}
Oscillator strengths and radiative transition probabilities for dipole allowed fine structure levels are obtained for Silike ions: Si I, S III, Ar V and Ca VII from multiplet line strengths calculated for the Opacity Project and using the observed energy levels. The radiative calculations are carried out in the close-coupling approximation employing the R-matrix method as developed for the Opacity Project. There are relatively little previous data available for the transition probabilities of these ions. Comparisons of the present oscillator strengths with available experimental values exhibit good agreement in general.
\end{abstract}

\section{Introduction}

The Opacity Project has produced large quantities of accurate radiative data, energy levels, photoionization cross sections and oscillator strengths, for essentially all astrophysically abundant atoms and ions using $a b$ initio methods based on the close coupling approximation and the R-matrix method [1]. Prior to the Opacity Project (hereafter OP), relatively few oscillator strengths were known for most of the elements and various ionization stages. The OP work therefore has resulted in making available huge amounts of radiative data. The OP calculations however have been carried out in the $L S$ coupling approximation and the fine structures within the multiplet transitions are not considered. For the calculation of stellar opacities (the stated aim of the OP), this is a valid assumption in most regions of stellar plasmas as the fine structure is almost entirely subsumed by plasma broadening effects and a redistribution of oscillator strengths within the multiplets does not significantly affect the total opacities.

On the other hand for most other spectroscopic applications the transitions of interest are usually observed to be between individual fine structure levels and it is necessary to obtain the corresponding oscillator strengths. In a previous work on the Si-like ions [2] we have discussed the calculations and presented selected results for photoionization cross sections and LS multiplet $f$-values. In this paper we extend the OP work to calculate the fine structure $f$-values for the individual transitions within the multiplets through an algebraic transformation of the $L S$ line strengths to fine structure components. In addition, the calculated $f$-values are obtained using observed energy levels and are thus an improvement over the original OP data that uses the calculated energies. Although the difference between the observed and the calculated energies is small in general, the $f$-values for closely spaced transitions can be significantly affected. 
Detailed comparisons are made with available oscillator strengths from a variety of experimental (laser fluorescence, beam-foil etc.) sources.

In our OP calculations [2], we had considered all transitions between bound states up to $n$ $\leq 10$ and $l \leq 5$ (i.e. all resulting $L S$ multiplets). In the present work we report only a subset of the oscillator strengths data, derived from the earlier calculations, as the extent of the present work is constrained by the availability of the observed energy levels, since these are required for the recalculations of the fine structure $f$-values. Thus, although we consider a large number of transitions, a considerable body of the original OP data is stjll unprocessed.

As there is relatively little previous data, the present work is aimed at providing a comprehensive set of oscillator strengths for many practical laboratory and astrophysical applications.

\section{Summary of theoretical work}

The calculations for the $L S$ oscillator strengths have been carried out in the close coupling (CC) approximation employing the R-matrix method. The CC expansion for the wavefunction of each ion consists of different sets of lowest states of the core ion such as 8-state CC expansion for Si I, 16-state CC expansion for S IV, 13-state CC expansion for Ar V, and 18-state CC expansion for Ca VII as given in Table I. More details about the spectroscopic and corre-

Table I. Ion core states in the CC expansion of the four elements of the Si-like ions; $N$ is the number of states included

\begin{tabular}{|c|c|c|}
\hline Ion & $N$ & States \\
\hline Sj II & 8 & $3 s^{2} 3 p\left({ }^{2} P^{\circ}\right), 3 s 3 p^{2}\left({ }^{4} P,{ }^{2} D\right), 3 s^{2} 4 s\left({ }^{2} S\right), 3 s 3 p^{2}\left({ }^{2} S\right), 3 s^{2} 3 d\left({ }^{2} D\right), 3 s^{2} 4 p\left({ }^{2} P^{0}\right), 3 s 3 p^{2}\left({ }^{2} P\right)$ \\
\hline S IV & 16 & 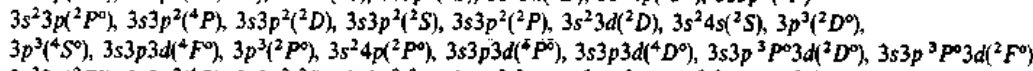 \\
\hline Ar VI & 13 & $\begin{array}{l}3 s^{2} 3 p\left(^{2} P^{\circ}\right), 3 s 3 p^{2}\left({ }^{4} P\right), 3 s 3 p^{2}\left({ }^{2} D\right), 3 s 3 p^{2}\left({ }^{2} S\right), 3 s 3 p^{2}\left({ }^{2} P\right), 3 s^{2} 3 d\left({ }^{2} D\right), 3 p^{3}\left({ }^{2} D^{\circ}\right), 3 p^{3}\left({ }^{4} S^{\circ}\right) \\
3 s 3 p 3 d\left({ }^{4} F^{\circ}\right), 3 p^{3}\left({ }^{2} p^{\circ}\right), 3 s 3 p 3 \mathrm{~d}\left({ }^{4} P^{\circ}\right), 3 s 3 p 3 \mathrm{~d}\left({ }^{4} D^{\circ}\right), 3 s 3 p^{3} p^{0} 3 d\left({ }^{2} D^{\circ}\right)\end{array}$ \\
\hline Ca VII & 18 & 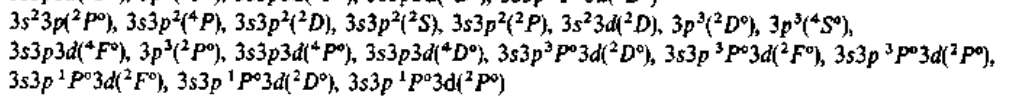 \\
\hline
\end{tabular}

lation configurations and comparison of energy values can be found in Ref. [2]. The oscillator strength or the $f$-value for a bound-bound transition from state i to state $\mathrm{f}$ is given by

$$
f_{\mathrm{if}}=\frac{E_{\mathrm{fi}}}{3 g_{\mathrm{i}}} S
$$

where $E_{f i}=E_{f}-E_{i}$ is the transition energy, $g_{\mathrm{i}}=\left(2 S_{\mathrm{i}}+1\right)\left(2 L_{\mathrm{j}}+1\right)$ is the statistical weight of the initial state in $L S$ coupling or $g_{i}=\left(2 J_{i}+1\right)$ in fine structure, and $S$ is the line strength (energies are in Rydberg unit throughout unless specified otherwise). In terms of dipole length and velocity operators

$$
\mathbf{D}_{\mathrm{L}}=\sum_{n} \mathbf{r}_{n}, \quad \mathbf{D}_{\mathrm{V}}=-2 \sum_{n} \nabla_{n}
$$

where the summation is over all atomic electrons, the line strength is given by 
$\mathbf{S}_{\mathbf{L}}=\left|\left\langle\psi_{\mathrm{f}}\left\|\mathbf{D}_{\mathrm{L}}\right\| \psi_{\mathrm{i}}\right\rangle\right|^{2}$,

in the "length form" and

$$
S_{\mathrm{v}}=E_{\mathrm{fi}}^{-2}\left|\left\langle\psi_{\mathrm{f}}\left\|\mathbf{D}_{\mathrm{v}}\right\| \psi_{\mathrm{j}}\right\rangle\right|^{2}
$$

(3a)

in the "velocity form" respectively. For exact wave functions

$\Psi, S_{\mathrm{L}}=S_{\mathrm{V}}$

The radiative transition probability, $A_{f i}$ or the A-value, from a higher state $f$ to a lower state $\mathrm{i}$ is related to oscillator strength $f_{\mathrm{if}}$ in atomic unit as

$$
A_{\mathrm{fi}}(\text { a.u. })=\frac{1}{2} \alpha^{3} \frac{g_{1}}{g_{\mathrm{f}}} E_{\mathrm{fi}}^{2} f_{\mathrm{fi}},
$$

where $\alpha$ is the fine structure constant and $g_{f}$ is the statistical weight of the final state; in c.g.s. unit of time $A_{\mathrm{fi}}$ is given by

$$
A_{\mathrm{fi}}\left(\mathrm{sec}^{-1}\right)=\frac{A_{\mathrm{fi}}(\text { a.u. })}{\tau_{0}}
$$

where $\tau_{0}=2.419 \times 10^{17}$ sec is the atomic unit of time. The total radiative probability for the state $\mathrm{f}$ is

$$
A_{\mathrm{f}}=\sum_{i} A_{\mathrm{fi}}
$$

and the lifetime of the state is obtained as

$$
\tau_{\mathrm{f}}=1 / A_{\mathrm{f}} .
$$

In the present work, the line strength $S$ of a transition in $L S$ multiplet is obtained from the oscillator strengths, using eq. (1), of the OP data for Si-like ions Si I, S III, Ar V and Ca VII and are split into fine structure transitions using standard algebraic transformation factors [3]. Correction to transition energies is now made by using observed energies to obtain the fine structure oscillator strengths and $A$-values. This improves the accuracy of the $f$-values since the spectroscopic energies are known to high precision. From large number of oscillator strengths in LS coupling obtained for the OP [2] such as 3149 values with 218 LS bound states of Si I, 3973 values with 236 bound states of S III, 7863 values with 342 bound states of Ar V and 16961 values with 497 bound states of Ca VII, with $n \leq 10$ and $l \leq 5$, we choose a small number of transitions for those states only that have been experimentally observed. Fine structure splitting is carried out for $f$-values calculated in length form only. The reason is that they are more accurate in the R-matrix calculations since the matrix elements for length form are weighted more towards the asymptotic region where the wave functions are better represented than the inner region [2].

\section{Results}

As the main aim of this presentation is to make available an extensive amount of transition probability data, derived from the OP, it is necessary to also ascertain the uncertainties in the theoretical calculations as compared with the most recent and reliable measurements in so far as possible. For the Si sequence there exist a few accurate sets of measurements by O'Brian and Lawler [4], Becker et al. [5], Berry et al. [6], Livingston et al. [7] and several others [8, 9]. In our 
previous work [2], we have already compared the $L S$ multiplet $f$-values from the OP calculations with available data. Whereas the present work is concerned with the more detailed fine structure transitions, in this section we compare the corresponding $f$-values with available experimental ones, primarily from sources cited above. We also compare the lifetimes of a number of excited states with the available measured values.

Table II presents the OP energy values of $L S$ states along with the available observed values [10] for comparison. In accordance with the standard spectroscopic convention (e.g. in NIST tables) the table labels the energy order of states such that excited states of a particular SL $\pi$ with even parity are represented by ascending alphabets and with odd parity by descending alphabets. The same notation for energy order will be used later in Tables III, IV and V. It should be noted that all $f$-values and $A$-values in the present work for $L S$ multiplet transitions, as well as for fine structure components, are obtained using observed spectroscopic energies. The calculated energies agree within 3\% of observed values except some excited states of Si I, as discussed in Ref. [2]. From the point of view of observational spectroscopy this difference between the calculated and observed energies is significant. However, one might note that the OP calculations are the first $a b$ initio calculations that obtain the spectroscopic data in a complete and self-consistent manner for an arbitrary large number of bound states of the atom or ion. As such, the number of transitions considered, even within the $L S$ multiplet scheme, is very large. Nonetheless an obvious improvement over the OP results, implemented in the present work, is to employ the observed energies.

Detailed comparisons of the computed $f$-values with experimental measurements are presented in Table III. The first row lists the total $L S$ multiplet transition, the corresponding oscillator strengths in the length and the velocity forms, and the experimental value. The fine structure $f$-values, obtained from the length form, are then compared individually with the experimental values. The calculated and measured $A$-values are also given for Si I. Si I experimental data are reported by O'Brian and Lawler [4], who have measured a number of transitions using laser-induced fluorescence technique which apparently has very low uncertainties, and by Becker et al. [5] who used beam-foil technique. The error bars for measured values of Si I by O'Brian and Lawler are approximate since they have been converted from $\pm \log _{10}(g f)$. Comparison of the present data with the measurements of O'Brian and Lawler [4] provides an accurate indicator of the overall uncertainties in the theoretical calculations. We find that the overall agreement between the two sets of data for the 26 fine structure transitions reported is within 5-10\%, with the exception of some very weak transitions, e.g. $a^{1} D_{2}^{e}-y^{1} P_{1}^{o}$ This is a general feature of most of the theoretical calculations, i.e. small $f$-values with $|f| 1<0.01$, tend to involve significant amounts of calculation in the dipole matrix elements with resultant loss of accuracy in the final value. Agreement of the present $f$-values with the limited measured values by Becker et al. [5] is also good except for the same weak transition. We may thus estimate the uncertainty in the present $f$-values for Si I to be less than $10 \%$ for transitions with $\mid f>0.01$.

For S III the available experimental data is much more sparse and from a number of different sources, all using 
Table II. Comparison of calculated bound state energies of Si I, S III, Ar V and Ca VII

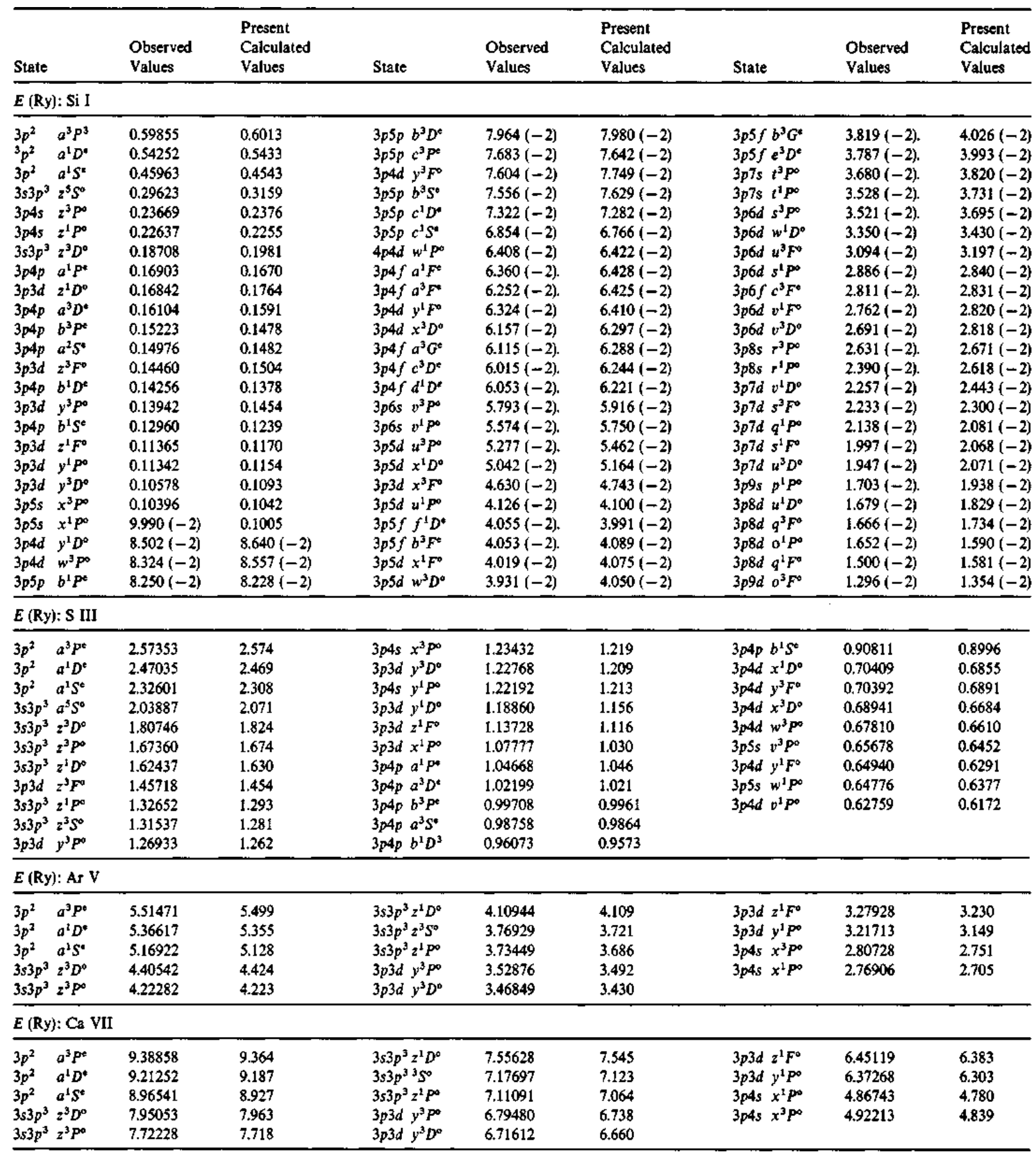

The negative sign for the energy values has been omitted for convenience. Observed energies are from Martin and Zalubus (1983) and with "." at the end from the compilation by C. Moore (1949) for Si I, from Johansson et al. (1992) except ${ }^{5} S^{\circ}$ which is from Martin et al. (1990) for S III, from Kelly (1987) for Ar V, and from Sugar and Corliss (1985) for Ca VII. 
Table III. Comparison of the present oscillator strengths or the f-values, and transition probabilities $A_{\mathrm{fi}}$ in sec $^{-1}$. with the observed values. $f_{\mathrm{L}}$ and $f_{\mathrm{v}}$ are the calculated oscillator strengths in length and velocity forms respectively

\begin{tabular}{|c|c|c|c|c|c|c|c|c|c|}
\hline \multirow[b]{3}{*}{ Transition } & \multirow[b]{3}{*}{ Multiplet } & \multirow[b]{3}{*}{$g_{1}$} & \multirow[b]{3}{*}{$g_{\mathrm{r}}$} & \multirow{3}{*}{$\begin{array}{l}W L \\
(\mathrm{~A})\end{array}$} & $\mathrm{Si} \mathrm{I}$ & & \multirow{3}{*}{$f_{\text {Expt. }}$} & \multirow{3}{*}{$\begin{array}{l}A_{t 1} \\
\text { Present }\end{array}$} & \multirow{3}{*}{$\begin{array}{l}A_{\text {fi }} \\
\text { Expt. }\end{array}$} \\
\hline & & & & & $f_{1}$ & $f_{v}$ & & & \\
\hline & & & & & \multicolumn{2}{|c|}{ Present } & & & \\
\hline \multirow[t]{9}{*}{$3 p^{2}-3 p 4 s$} & $a^{3} P^{e}-z^{3} P^{0}$ & 9. & 9 & & 0.236 & 0.216 & $0.211^{*}$ & & \\
\hline & & 5 & 5 & 2518.87 & 0.1767 & & $0.1592 \pm 0.008,0^{\bullet} 0.155^{b}$ & $1.86(8)$ & $1.68(8)^{n}$ \\
\hline & & 5 & 3 & 2531.32 & 0.0586 & & $0.0520 \pm 0.0027^{\circ}$ & $1.02(8)$ & $9.04(7)^{\circ}$ \\
\hline & & 3 & 5 & 2509.65 & 0.0985 & & $0.0859 \pm 0.00433^{\mathrm{a}} 0.098^{\mathrm{b}}$ & $6.27(7)$ & $5.47(7)^{\circ}$ \\
\hline & & 3 & 3 & 2522.00 & 0.0588 & & $0.0522 \pm 0.0026^{\circ}$ & $6.18(7)$ & $5.49(7)^{*}$ \\
\hline & & 3 & 1 & 2526.89 & 0.0783 & & $0.0708 \pm 0.0034^{a}$ & $2.46(8)$ & $2.22(8)^{\circ}$ \\
\hline & & 1 & 3 & 2517.11 & 0.2358 & & $0.2104 \pm 0.01^{*}$ & $8.29(7)$ & $7.40(7)^{2}$ \\
\hline & $a^{1} D^{*}-z^{1} P^{\circ}$ & 5 & 3 & 2881.58 & 0.194 & 0.179 & $0.162 \pm 0.008,0.170^{\circ}$ & & \\
\hline & $a^{1} S^{6}-z^{1} P^{0}$ & 1 & 3 & 3905.52 & 0.103 & 0.087 & $0.091 \pm 0.005,^{*} 0.098^{b}$ & & \\
\hline \multirow[t]{11}{*}{$3 p^{2}-3 p 3 d$} & $a^{3} p^{e}-y^{3} p^{0}$ & 9 & 9 & & 0.054 & 0.054 & $0.0513^{*}$ & & \\
\hline & & $s$ & 5 & 1990.59 & 0.0402 & & $0.0390 \pm 0.002^{*}$ & $6.78(7)$ & $6.57(7)^{2}$ \\
\hline & & 5 & 3 & 1987.98 & 0.0134 & & $0.0129 \pm 0.0006^{\circ}$ & $3.78(7)$ & $3.65(7)^{n}$ \\
\hline & & 3 & 5 & 1984.82 & 0.0224 & & $0.0214 \pm 0.001^{\circ}$ & $2.28(7)$ & $2.18(7)^{4}$ \\
\hline & & 3 & 3 & 1982.23 & 0.0135 & & $0.0122 \pm 0.0006^{4}$ & $2.29(7)$ & $2.07(7)^{4}$ \\
\hline & & 3 & 1 & 1980.81 & 0.0179 & & $0.0170 \pm 0.0008^{2}$ & $9.17(7)$ & $8.70(7)^{\circ}$ \\
\hline & & 1 & 3 & 1979.21 & 0.0539 & & $0.0491 \pm 0.002^{\circ}$ & $3.06(7)$ & $2.79(7)^{\circ}$ \\
\hline & $a^{1} D^{e}-y^{1} P^{\circ}$ & 5 & 3 & 2122.99 & 0.0056 & 0.0057 & $0.0029 \pm 0.0005, ; 0.0036^{\circ}$ & & \\
\hline & $a^{1} D^{*}-z^{1} D^{\circ}$ & 5 & 5 & 2435.15 & 0.041 & 0.041 & $0.0394 \pm 0.002^{x}$ & & \\
\hline & $a^{1} D^{e}-z^{1} F^{\circ}$ & 5 & 7 & 2124.12 & 0.358 & 0.336 & $0.282 \pm 0.015^{*}$ & & \\
\hline & $a^{1} S^{e}-y^{1} P^{\circ}$ & 1 & 3 & 2631.28 & 0.409 & 0.409 & $0.330 \pm 0.017, ; 0.355^{b}$ & & \\
\hline \multirow[t]{9}{*}{$3 p^{2}-3 s 3 p^{3}$} & $a^{3} P^{4}-z^{3} D^{\circ}$ & 9 & 15 & & 0.052 & 0.053 & $0.056^{\circ}$ & & \\
\hline & & 5 & 7 & 2219.16 & 0.0437 & & $0.0469 \pm 0.0023^{\circ}$ & $4.23(7)$ & $4.54(7)^{e}$ \\
\hline & & 5 & 5 & 2220.51 & $7.79(-3)$ & & $8.05(-3) \pm 0.4(-3)^{\mathrm{t}}$ & $1.06(7)$ & $1.09(7)^{2}$ \\
\hline & & 5 & 3 & 2221.38 & $5.19(-4)$ & & $4.63(-4) \pm 0.6(-4)^{\circ}$ & $1.17(6)$ & $1.05(6)^{\circ}$ \\
\hline & & 3 & 5 & 2213.34 & 0.0391 & & $0.0422 \pm 0.002^{2}$ & $3.20(7)$ & $3.45(7)^{4}$ \\
\hline & & 3 & 3 & 2214.20 & 0.0130 & & $0.0134 \pm 0.0007^{\circ}$ & $1.77(7)$ & $1.81(7)^{\circ}$ \\
\hline & & 1 & 3 & 2210.43 & 0.0522 & & $0.0575 \pm 0.003^{\circ}$ & $2.38(7)$ & $2.62(7)^{\circ}$ \\
\hline & & & & & \multicolumn{3}{|l|}{ S III } & & \\
\hline & & & & & $f_{L}$ & $f_{v}$ & & & \\
\hline & Multiplet & $\varepsilon_{i}$ & $g_{t}$ & & \multicolumn{2}{|c|}{ Present } & Expt. & & \\
\hline \multirow[t]{3}{*}{$3 p^{2}-3 s 3 p^{3}$} & $a^{3} P^{3}-z^{4} D^{a}$ & 9 & 15 & & 0.0246 & 0.0217 & $0.022 \pm 0.002 i^{\mathrm{z}}, 0.022^{\mathrm{b}}$ & & \\
\hline & $a^{3} P^{0}-z^{3} P^{0}$ & 9 & 9 & & 0.0425 & 0.0374 & $0.036^{\circ}$ & & \\
\hline & $a^{1} D^{e}-z^{1} D^{\circ}$ & 15 & 15 & & 0.0216 & 0.0194 & $0.0167 \pm 0.005,0.99 \pm 0.10^{c}$ & & \\
\hline $3 p^{2}-3 p 3 d$ & $a^{3} P^{c}-y^{3} D^{\circ}$ & 9 & 15 & & 1.637 & 1.604 & $0.96 \pm 0.19^{d}$ & & \\
\hline $3 p 4 p-3 p 4 d$ & $a^{3} D^{*}-y^{3} F^{0}$ & 15 & 21 & & 0.908 & 0.844 & $0.685 \pm 0.05^{n}$ & & \\
\hline \multirow[t]{4}{*}{$3 p^{2}-3 p 4 s$} & $a^{1} D^{c}-y^{1} P^{o}$ & 15 & 9 & & 0.0930 & 0.0930 & $0.07 \pm 0.04^{4}$ & & \\
\hline & $a^{1} S^{e}-y^{1} P^{\infty}$ & 1 & 9 & & 0.0662 & 0.0630 & $0.08 \pm 0.05^{d}$ & & \\
\hline & & & & & Ar V & & & & \\
\hline & & & & & $f_{L}$ & $f_{v}$ & \multirow{2}{*}{$\begin{array}{l}f \\
\text { Expt. } \\
\end{array}$} & & \\
\hline Transition & Multiplet & $g_{i}$ & $g_{t}$ & & \multicolumn{2}{|c|}{ Present } & & & \\
\hline $3 p^{2}-3 s 3 p^{3}$ & $a^{3} P^{0}-z^{3} P^{0}$ & 9 & 9 & & 0.061 & 0.059 & $0.057 \pm 0.002^{\circ}$ & & \\
\hline
\end{tabular}

beam-foil technique. The reported measurements are for $L S$ mulitplet transitions between a few triplet and singlet states as given in Table III. The computed $f$-value for the transition $a^{3} P^{e}-z^{3} D^{\circ}$ is in very good agreement with both the Berry et al. [6] and Livingston et al. [7], nearly within experimental uncertainties. For the transition $a^{1} D^{e}-z^{1} D^{\circ}$, present results are in closer agreement with the Berry et al. value than with Irwin et al. [8]. However, for the $a^{3} P^{e}-y^{3} D^{\circ}$ transition our results differ considerably with the value reported by Ryan et al. [9]. For the remaining 
transitions

Table III. (continued)

\begin{tabular}{|c|c|c|c|c|c|c|}
\hline \multirow[b]{3}{*}{ Transition } & \multirow[b]{3}{*}{ Mulitiplet } & \multirow[b]{3}{*}{$\theta_{\mathrm{i}}$} & \multirow[b]{3}{*}{$\theta_{\mathrm{f}}$} & \multicolumn{2}{|l|}{$\mathrm{Ca}$ VII } & \multirow{3}{*}{$\begin{array}{l}f \\
\text { (EB) }\end{array}$} \\
\hline & & & & $f_{\mathrm{L}}$ & $f_{v}$ & \\
\hline & & & & \multicolumn{2}{|c|}{ Present } & \\
\hline \multirow[t]{10}{*}{$3 p^{2}-3 s 3 p^{3}$} & $a^{3} P^{*}-z^{3} p^{0}$ & 9 & 9 & 0.0680 & 0.0648 & 0.064 \\
\hline & & 5 & 5 & 0.0507 & & 0.0502 \\
\hline & & 5 & 3 & 0.0169 & & 0.0135 \\
\hline & & 3 & 5 & 0.0285 & & 0.0210 \\
\hline & & 3 & 3 & 0.0171 & & 0.0210 \\
\hline & & 3 & 1 & 0.0228 & & 0.0220 \\
\hline & & 1 & 3 & 0.0691 & & 0.0631 \\
\hline & $a^{1} D^{4}-z^{1} p^{\infty}$ & 5 & 3 & 0.247 & 0.243 & 0.236 \\
\hline & $a^{1} D^{e}-z^{1} D^{0}$ & 5 & 5 & 0.0889 & 0.0853 & 0.096 \\
\hline & $a^{1} s^{6}-z^{1} P^{a}$ & 1 & 3 & 0.174 & 0.165 & 0.191 \\
\hline & $a^{1} D^{6}-z^{1} F^{0}$ & 5 & 7 & 0.959 & 0.959 & 0.896 \\
\hline \multirow[t]{2}{*}{$3 p^{2}-3 p 4 s$} & $a^{1} D^{*}-x^{1} P^{0}$ & 5 & 3 & 0.139 & 0.135 & 0.133 \\
\hline & $a^{1} S^{4}-x^{1} P^{0}$ & 1 & 3 & 0.114 & 0.108 & 0.120 \\
\hline
\end{tabular}

Si I: ' O'Brian and Lawler (1991); ${ }^{\circ}$ Becker et al. (1980).

S III: ' Berry et al. (1970); ${ }^{\circ}$ Livingston et al. (1976); ${ }^{\circ}$ Irwin et al. (1973); ${ }^{d}$ Ryan et al. (1989).

Ar V: " Irwin et al. (1973).

Ca VII: EB, Biemont (1986).

we find differences with the earlier beam-foil results at about $10-20 \%$ level; however, the number of comparisons is too few to ascertain any definite limit on the uncertainties in the S III calculations.

For $\mathrm{Ar} \mathrm{V}$, there is only one available measurement for the $a^{3} P^{e}-z^{3} P^{\circ}$ transition and the present $f$-value is in good agreement with it. To our knowledge, there are no experimental measurements for Ca VII. As discussed in Ref. [2], oscillator strengths for these two ions are expected to have good accuracy since they have low discrepancy (about 5\%) between the length and the velocity forms for most of the transitions. In Ref. [2], we carried out detailed comparisons of the oscillator strengths for these four ions with available theoretical works. Earlier detailed calculations include six-state close coupling calculations of Mendoza and Zeippen [11] for a few transitions in Si I, atomic structure calculations by Ho and Henry [12] also for a limited transition in S III. Both works correspond to LS multiplet oscillator strengths. There seem to be no theoretical calculations for Ar V oscillator strengths. Atomic structure calculations for fine structure oscillator strengths in Ca VII was carried out by Biemont [13] for a number of transitions. Comparison of the present results with his values for some transitions are made in Table III and both calculated values agree well with each other.

In addition to direct comparison of calculated oscillator, strengths with the experimental values, another indicator of the overall uncertainties may be obtained by considering the lifetimes of excited states, as the calculations of lifetimes involves a number of transitions through which the given state may undergo radiative decay. Table IV presents and compares calculated lifetimes of a number of excited states for which measured values are available. These lifetimes are obtained from the sum, eq. (7), of radiative transition probabilities, or the $A$-values, of the dipole allowed states given in Table V. Notation for states are the same as Table II. The quoted experimental uncertainties are given within parentheses next to the values. Measured lifetimes for a set of fine structure levels are available mainly for Si I [4, 14], with which we compare our results. The 
present calculated values for Si I are in fair agreement with the laser excitation and time resolved detection measurement by Bergstrom et al. [14], the difference between the calculated and measured values ranging from $2 \%$ for $b^{3} D_{3}^{e}$ to $28 \%$ for $c^{3} P^{e}$. The agreement between the calculated and measured values using laser-induced fluorescence method by O'Brian and Lawler [4] is good in general except for some levels such as $u^{l} P^{o}{ }_{1}^{o}, w^{3} P_{2,1,0}^{o}, y^{1} D_{2}^{\circ}, y^{3} D_{1,2,3}^{\circ}$ The difference is large especially for $\mathrm{u}^{1} \mathrm{P}^{0}{ }_{1}, w^{3} P^{\circ}{ }_{2,1,0}$. As can be seen from Table $\mathrm{V}$ that the lifetime contributions to $u^{1} \mathrm{P}^{0}{ }_{1}$ come from radiative decay to dipole allowed states $a^{1} S^{e}, b^{1} s^{e} c^{1} s^{e}, a^{1} P^{e}$, $b^{1} P^{e}, a^{1} d^{e}, b^{1} D^{e}, c^{l} D^{e}, d^{1} D^{e}$ and $e^{1} D^{e}$. The number of routes of radiative decay is limited in this work by the number of observed energy levels. It could be possible that inclusion of contributions from higher excitated states such as of ${ }^{1} S^{e},{ }^{1} P^{e}$.would have increased the total transition probability and hence reduce the lifetime. The forbidden transitions usually have very small contributions to the total transition probablity. The opposite is the case for $w^{3} P_{2}^{\circ}, 1,0$ levels where the calculated lifetimes are much shorter than those measured by O'Brian and Lawler. For $w^{3} P^{\circ}$ states, the dominant contributions come from decay to levels of the terms $a^{3} S^{e}, a^{3} P^{e}$ and $b^{3} P^{e}$, resulting in a large total transition probability and smaller lifetime for each $w^{3} P^{\circ}$ level. The reason for this difference is not very obvious from the present calculations since the calculated oscillator strengths from transitions among the above levels do not have a large discrepancy between the length and the velocity forms.

For S III and Ar V, lifetimes are given for $L S$ excited states. S III lifetimes have been measured by beam-foil technique by various investigators. Present values for lifetime compares well with Berry et al. [6], Livingston et al. [7], Dumont et al. [15] and Irwin and Livingston [16]. The calculated lifetime of Ar V states agree well with those measured by Livingston et al. [17] except for states $y^{3} P^{\circ}, z^{1} F^{\circ}$. Calculated lifetime agree well with the single measurement 


\begin{tabular}{|c|c|c|c|c|c|c|c|c|}
\hline \multirow[b]{2}{*}{ State } & \multicolumn{2}{|l|}{$t(\mathrm{~ns})$} & \multirow[b]{2}{*}{ State } & \multicolumn{2}{|l|}{$\tau(n s)$} & \multirow[b]{2}{*}{ State } & \multicolumn{2}{|l|}{$\tau$ (ns) } \\
\hline & Cad. & Obs. & & Cal. & Obs. & & Cal. & Obs. \\
\hline \multicolumn{9}{|l|}{ Si I } \\
\hline$b^{3} S_{1}^{4}$ & 167.6 & $180^{3}$ & $x^{3} P_{0}^{\circ}$ & 13.7 & $13.8(0.7)^{6}$ & $z^{3} D_{2}^{\circ}$ & 23.5 & $22.0(1.1)^{b}$ \\
\hline$z^{1} p_{1}^{*}$ & 3.64 & $4.3(0.2)^{b}$ & $x^{3} P_{1}^{0}$ & 13.6 & $12.6(0.6)^{b}$ & $z^{3} D_{3}^{0}$ & 23.6 & $22,0(1.1)^{b}$ \\
\hline$y^{1} P_{1}^{0}$ & 6.64 & $8.5(0.4)^{b}$ & $x^{3} P_{2}^{0}$ & 13.4 & $13.4(0.7)^{b}$ & $y^{3} D_{1}^{0}$ & 3.06 & $4.2(0.2)^{b}$ \\
\hline$x^{1} P_{1}^{0}$ & 8.69 & $8.8(0.4)^{b}$ & $w^{3} P_{2}^{0}$ & 23.5 & $37.2(1.9)^{b}$ & $y^{3} D_{2}^{6}$ & 3.07 & $4.0(0.2)^{b}$ \\
\hline$w^{1} p_{i}^{0}$ & 15.7 & $10.8(0.5)^{\mathrm{b}}$ & $w^{3} P_{2}^{0}$ & 23.3 & $32.4(1.6)^{b}$ & $y^{3} D_{3}^{\circ}$ & 3.09 & $4.1(0.2)^{b}$ \\
\hline$u^{1} P_{1}^{0}$ & 29.6 & $13.4(0.7)^{\mathrm{b}}$ & $w^{3} P_{0}^{0}$ & 23.1 & $31.9(1.6)^{b}$ & $x^{3} D_{3}^{0}$ & 4.58 & $4.8(0.3)^{b}$ \\
\hline$c^{3} P_{0}^{*}$ & 125.7 & $148^{n}$ & $u^{3} P_{2}^{\infty}$ & 11.6 & $1.1(0.6)^{b}$ & $x^{3} D_{2}^{0}$ & 4.57 & $4.8(0.3)^{b}$ \\
\hline$c^{3} p_{1}^{4}$ & 127.1 & $168^{*}$ & $u^{3} P_{1}^{0}$ & 11.5 & $11.7(0.6)^{b}$ & $x^{3} D_{1}^{0}$ & 4.55 & $5.7(0.3)^{b}$ \\
\hline$c^{3} P_{2}^{e}$ & 122.6 & $152^{2}$ & $u^{3} P_{0}^{0}$ & 11.4 & $12.1(0.6)^{b}$ & $w^{3} D_{3}^{0}$ & 7.69 & $8.5(0.4)^{b}$ \\
\hline$z^{3} P_{0}^{0}$ & 4.07 & $4.5(0.2)^{\mathrm{b}}$ & ${ }^{1} D_{2}^{0}$ & 21.5 & $22.4(1.1)^{b}$ & $w^{3} D_{2}^{0}$ & 7.67 & $8.1(0.4)^{b}$ \\
\hline $2^{3} P_{1}^{0}$ & 4.06 & $4.5(0.2)^{b}$ & $y^{1} D_{2}^{0}$ & 36.6 & $44.5(2.2)^{b}$ & $w^{3} D_{t}^{0}$ & 7.67 & $16.1(0.8)^{b}$ \\
\hline$z^{3} P_{2}^{0}$ & 4.02 & $4.5(0.2)^{0}$ & $b^{3} D_{i}^{c}$ & 253.2 & $266^{\circ}$ & $z^{1} F_{3}^{0}$ & 2.61 & $3.3(0.2)^{b}$ \\
\hline$y^{3} p_{2}^{0}$ & 11.0 & $11.4(0.6)^{b}$ & $b^{3} D_{2}^{*}$ & 262.0 & $253^{\circ}$ & $y^{1} F_{3}^{0}$ & 6.02 & $6.2(0.3)^{b}$ \\
\hline$y^{3} P_{1}^{0}$ & 10.9 & $11.5(0.6)^{b}$ & $b^{3} D_{3}^{e}$ & 255.4 & $260^{\circ}$ & $x^{1} F_{3}^{\circ}$ & 11.3 & $10.2(0.5)^{b}$ \\
\hline$y^{3} P_{0}^{0}$ & 10.9 & $11.7(0.6)^{6}$ & $z^{3} D_{t}^{0}$ & 23.4 & $22.0(1.1)^{b}$ & $v^{1} F_{3}^{0}$ & 18.9 & $19.2(1.0)^{b}$ \\
\hline \multicolumn{9}{|l|}{ S III } \\
\hline$a^{3} S^{4}$ & 1.83 & $2.05(0.1)^{\circ}$ & $x^{3} p^{0}$ & 0.813 & $1.1(0.1)^{2}$ & $z^{1} p^{0}$ & 0.154 & $0.18(0.02)^{d}$ \\
\hline$b^{3} P^{e}$ & 2.10 & $2.0(0.1)^{6}$ & $z^{3} D^{\circ}$ & 14.6 & $16(1.0)^{\mathrm{b}}, 15.9(0.5)^{\mathrm{e}}$ & $z^{1} D^{\circ}$ & 8.07 & $10.4(0.3)^{a}$ \\
\hline$z^{3} P^{0}$ & 3.66 & $4.4(0.4)^{b}$ & $y^{3} D^{6}$ & 0.070 & $0.11(0.02){ }^{c} 0.12(0.03)^{d}$ & $a^{3} D^{*}$ & 2.01 & $2.58(0.2)^{*}$ \\
\hline$y^{3} P^{0}$ & 0.096 & $0.12(0.02)^{c}$ & & & & & & \\
\hline \multicolumn{9}{|l|}{ Ar V } \\
\hline$z^{1} p^{0}$ & 0.094 & $0.12(0.02)^{2}$ & $y^{3} P^{0}$ & 0.056 & $0.13(0.02)^{\star}$ & $z^{1} F^{\circ}$ & 0.034 & $0.06(0.02)^{2}$ \\
\hline$z^{3} p^{0}$ & 1.25 & $1.20(0.1)^{*} 1.33(0.05)^{b}$ & $2^{3} D^{\circ}$ & 0.036 & $0.03(0.01)^{4}$ & & & \\
\hline
\end{tabular}

Si I: " Bergstrom et al. (1989); ' O'Brian and Lawler (1991).

S HI: "Berry et al. (1970); "Livingston et al. (1976); " Dumont et al. (1978); " Irwin and Livingston (1974).

Ar V: ' Livingston et al. (1981); ' Irwin et al. (1973).

by Irwin et al. [8] also. The main point with respect to the lifetimes is that the present calculations yield a fairly complete set of oscillator strength data which, in turn, can be used to obtain lifetimes for a large number of states with uncertainties as indicated by the detailed comparisons in Table III. Even though agreement of the present calculated values of radiative lifetime of excited states varies in comparison with different measurements, the present calculations have been carried out in a theoretically and computationally-consistent manner for a large number of transitions.

Finally, we present all calculated dipole oscillator strengths $\left(f_{\text {if }}\right)$, line strengths $(S)$ and transition probabilities $\left(A_{\mathrm{fi}}\right.$ in $\left.\mathrm{sec}^{-1}\right)$ of the Si-like ions, Si I, S III, Ar V, and Ca VII in Tables Va, $\mathrm{b}, \mathrm{c}$, and $\mathrm{d}$ respectively. Each table lists the transitions among singlet states first, and then those among triplet states. The $f$-, $S$-, and $A$-values are presented for both the $L S$ multiplet and fine structure transitions. For a triplet-to-triplet transition, the first line corresponds to the transition in $L S$ multiplet and the following lines to its fine structure components. Conservation of total $L S$ multiplet line strength with the sum of fine structure components is checked for each $L S$ transition and the discrepancy is always found to be less than $0.01 \%$.

The notation for the states in Table V are the same as described for Table II. " $g$ " is the statistical weight factor of the initial or final state. Energies of initial and final states, and the transition energy between them are given for the fine structure transitions in the table. While the energy of each fine structure level is given in Rydberg units, the transition energy between the levels is given in terms of wavelength $(\lambda)$. However, for transitions in $L S$ coupling for the triplet states only transition energy in Rydberg is given between columns $E_{j}$ and $E_{f}$. It should be noted that the transition energies in terms of wavelengths may have uncertainties by a few Angstrom 
units. The observed energies [10] used for $f_{\text {if }}$ and $A_{\mathrm{fi}}$ values of the present work were obtained in units of $\mathrm{cm}^{-1}$ from the compilation of NIST. The transition wavelength in $\AA$ units is then obtained from the reciprocal of the transition energy in $\mathrm{cm}^{-1}$, thus introducing some numerical inaccuracy in $\lambda$ from the format of the energies written.

\section{Conclusion}

The Opacity Project calculations for $L S$ multiplets have been extended, using observed wavelengths, to calculate radiative transition probabilities for a large number of fine structure transitions. The present report provides an extensive set of data that compares well with the variety of available experimental measurements and is generally of high accuracy, with typical uncertainties of about $10 \%$. The data is expected to be useful in several astrophysical and laboratory applications.

\section{Acknowledgements}

The author would like to thank Professor Anil K. Pradhan for suggesting the work and comments. The work was carried out on the Cray Y-MP at the Ohio Supercomputer Center in Columbus, Ohio. A fellowship award by the College of Mathematical and Physical Sciences at the Ohio State University is gratefully acknowledged.

Table V(a). f-and A-values for Si I 


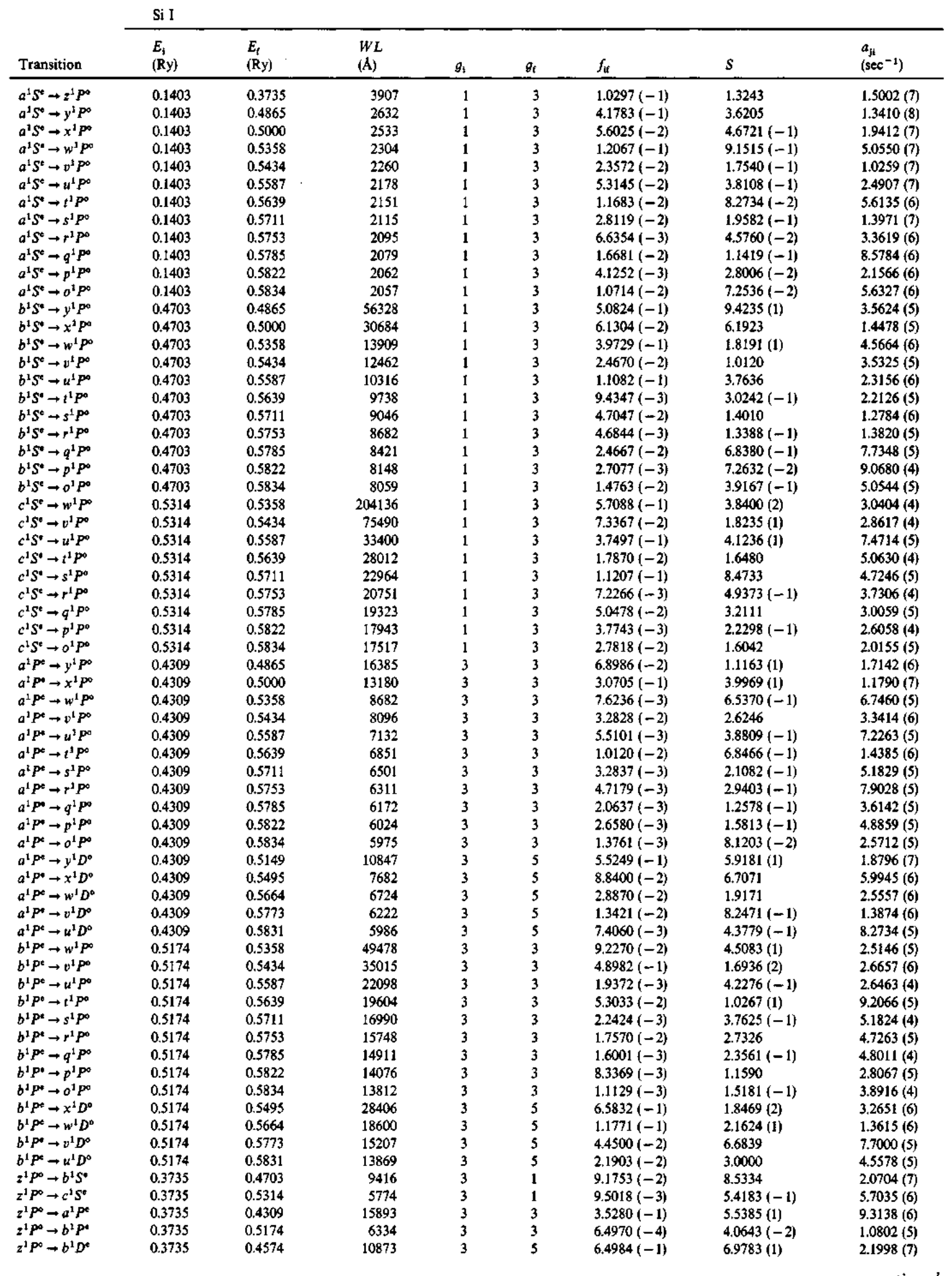

Table V(a). (continued) 


\begin{tabular}{|c|c|c|c|c|c|c|c|c|}
\hline \multirow[b]{2}{*}{ Transition } & \multicolumn{8}{|l|}{$\mathrm{Si} \mathrm{I}$} \\
\hline & $\begin{array}{l}E_{\text {i }} \\
\text { (Ry) }\end{array}$ & $\begin{array}{l}E_{\mathrm{r}} \\
\text { (Ry) }\end{array}$ & $\begin{array}{l}W L \\
(\AA)\end{array}$ & $g_{i}$ & $\theta_{\mathrm{f}}$ & $f_{\text {ir }}$ & $S$ & $\begin{array}{l}\mathfrak{a}_{j k} \\
\left(\sec ^{-1}\right)\end{array}$ \\
\hline$z^{1} p^{\circ} \rightarrow c^{1} D^{*}$ & 0.3735 & 0.5267 & 5950 & 3 & 5 & $3.3396(-2)$ & 1.9627 & $3.7745(6)$ \\
\hline$z^{1} P^{\infty} \rightarrow d^{1} D^{\circ}$ & 0.3735 & 0.5387 & 5519 & 3 & 5 & $3.1310(-3)$ & $1.7067(-1)$ & $4.1136(5)$ \\
\hline$z^{1} P^{\infty} \rightarrow e^{1} D^{\circ}$ & 0.3735 & 0.5586 & 4923 & 3 & 5 & $8.2376(-3)$ & $4.0055(-1)$ & $1.3600(6)$ \\
\hline$v^{1} p^{0} \rightarrow c^{1} S^{*}$ & 0.4865 & 0.5314 & 20307 & 3 & 1 & $4.0771(-2)$ & 8.1760 & $1.9789(6)$ \\
\hline$y^{1} p^{0} \rightarrow b^{1} p^{c}$ & 0.4865 & 0.5175 & 29472 & 3 & 3 & $1.1552(-4)$ & $3.3626(-2)$ & $8.8714(2)$ \\
\hline$y^{1} P^{0} \rightarrow c^{1} D^{e}$ & 0.4865 & 0.5267 & 22672 & 3 & 5 & $9.2770(-2)$ & 2.0775 (1) & $7,2216(5)$ \\
\hline$y^{1} P^{\infty} \rightarrow d^{1} D^{*}$ & 0.4865 & 0.5387 & 17472 & 3 & 5 & $8.1051(-1)$ & $1.3985(2)$ & $1.0627(7)$ \\
\hline$y^{1} P^{\infty} \rightarrow e^{1} D^{*}$ & 0.4865 & 0.5586 & 12633 & 3 & 5 & $1.5166(-2)$ & 1.8920 & $3.8037(5)$ \\
\hline$x^{1} P^{\infty} \rightarrow c^{1} S^{*}$ & 0.5000 & 0.5314 & 29064 & 3 & 1 & $8.2543(-2)$ & $2.3689(1)$ & $1.9561(6)$ \\
\hline$x^{1} P^{0} \rightarrow b^{1} p^{e}$ & 0.5000 & 0.5174 & 52373 & 3 & 3 & $5.4495(-1)$ & $2.8172(2)$ & $1.3252(6)$ \\
\hline$x^{1} P^{0} \rightarrow c^{1} D^{*}$ & 0.5000 & 0.5267 & 34164 & 3 & 5 & $7.6062(-1)$ & $2.5668(2)$ & $2.6074(6)$ \\
\hline$x^{1} P^{\infty} \rightarrow d^{1} D^{a}$ & 0.5000 & 0.5387 & 23586 & 3 & 5 & $1.2813(-1)$ & $2.9843(1)$ & 9.2194 (5) \\
\hline$x^{1} P^{\ominus} \rightarrow e^{1} D^{\natural}$ & 0.5000 & 0.5586 & 15547 & 3 & 5 & $4.1544(-2)$ & 6.3783 & $6.8800(5)$ \\
\hline$w^{1} P^{\circ} \rightarrow d^{1} D^{e}$ & 0.5358 & 0.5387 & 323226 & 3 & 5 & $1.3066(-1)$ & $4.1700(2)$ & $5.0076(3)$ \\
\hline$w^{1} P^{\circ} \rightarrow e^{1} D^{0}$ & 0.5358 & 0.5586 & 39972 & 3 & 5 & $1.8920(-1)$ & $7.4686(1)$ & $4.7401(5)$ \\
\hline$v^{1} P^{\infty} \rightarrow e^{1} D^{*}$ & 0.5434 & 0.5586 & 59990 & 3 & 5 & $\$ .1423$ & $6.7680(2)$ & $1.2702(6)$ \\
\hline$a^{1} D^{\epsilon} \rightarrow z^{1} P^{\circ}$ & 0.0574 & 0.3735 & 2882 & 5 & 3 & $1.9399(-1)$ & 9.2039 & $2.5957(8)$ \\
\hline$a^{1} D^{\circ} \rightarrow y^{1} P^{\circ}$ & 0.0574 & 0.4865 & 2124 & 5 & 3 & $5.6157(-3)$ & $1.9631(-1)$ & $1.3842(7)$ \\
\hline$a^{1} D^{*} \rightarrow x^{1} p^{\circ}$ & 0.0574 & 0.5000 & 2059 & 5 & 3 & $2.9588(-2)$ & 1.0027 & $7.7601(7)$ \\
\hline$a^{1} D^{\bullet} \rightarrow w^{1} P^{\circ}$ & 0.0574 & 0.5358 & 1905 & 5 & 3 & $2.4167(-3)$ & $7.5767(-2)$ & $7.4056(6)$ \\
\hline$a^{1} D^{e} \rightarrow v^{1} P^{\circ}$ & 0.0574 & 0.5434 & 1875 & 5 & 3 & $1.1126(-2)$ & $3.4335(-1)$ & $3.5187(7)$ \\
\hline$a^{1} D^{u}+u^{1} P^{\infty}$ & 0.0574 & 0.5587 & 1818 & 5 & 3 & $1.4490(-3)$ & $4.3361(-2)$ & $4.8739(6)$ \\
\hline$a^{1} D^{4} \rightarrow t^{1} P^{a}$ & 0.0574 & 0.5639 & 1799 & 5 & 3 & $5.4455(-3)$ & $1.6126(-1)$ & $1.8703(7)$ \\
\hline$a^{1} D^{e} \rightarrow s^{1} p^{0}$ & 0.0574 & 0.5711 & 1774 & 5 & 3 & $9.0182(-4)$ & $2.6335(-2)$ & $3.1854(6)$ \\
\hline$a^{1} D^{\mathrm{e}} \rightarrow r^{1} p^{\circ}$ & 0.0574 & 0.5753 & 1760 & 5 & 3 & $3.1248(-3)$ & $9.0505(-2)$ & $1.1220(7)$ \\
\hline$a^{1} D^{*} \rightarrow q^{1} P^{\circ}$ & 0.0574 & 0.5785 & 1749 & 5 & 3 & $5.9445(-4)$ & $1.7110(-2)$ & $2.1613(6)$ \\
\hline$a^{1} D^{*} \rightarrow p^{1} p^{0}$ & 0.0574 & 0.5822 & 1737 & 5 & 3 & $1.9572(-3)$ & $5.5946(-2)$ & $7.2152(6)$ \\
\hline$a^{1} D^{\mathrm{e}} \rightarrow o^{1} P^{\circ}$ & 0.0574 & 0.5834 & 1732 & 5 & 3 & $4.1091(-4)$ & $1.1718(-2)$ & $1.5220(6)$ \\
\hline$a^{1} D^{\circ} \rightarrow z^{1} D^{\circ}$ & 0.0574 & 0.4315 & 2436 & 5 & 5 & $4.1397(-2)$ & 1.6599 & $4.6535(7)$ \\
\hline$a^{1} D^{0} \rightarrow y^{1} D^{\circ}$ & 0.0574 & 0.5149 & 1992 & 5 & 5 & $2.4432(-3)$ & $8.0105(-2)$ & $4.1075(6)$ \\
\hline$a^{1} D^{e} \rightarrow x^{1} D^{\circ}$ & 0.0574 & 0.5495 & 1852 & 5 & 5 & $4.4436(-3)$ & $1.3545(-1)$ & $8.6434(6)$ \\
\hline$a^{1} D^{\circ} \rightarrow w^{1} D^{\circ}$ & 0.0574 & 0.5664 & 1790 & 5 & 5 & $3.6801(-3)$ & $1.0845(-1)$ & $7.6586(6)$ \\
\hline$a^{1} D^{4} \rightarrow v^{1} D^{\circ}$ & 0.0574 & 0.5773 & 1753 & 5 & 5 & $2.7655(-3)$ & $7.9784(-2)$ & $6.0052(6)$ \\
\hline$a^{1} D^{e} \rightarrow u^{1} D^{\circ}$ & 0.0574 & 0.5831 & 1733 & 5 & 5 & $2.0428(-3)$ & $5.8286(-2)$ & $4.5352(6)$ \\
\hline$a^{1} D^{e} \rightarrow 2^{1} F^{\circ}$ & 0.0574 & 0.4863 & 2125 & 5 & 7 & $3.6016(-1)$ & $1.2597(1)$ & $3.8006(8)$ \\
\hline$a^{1} D^{\circ} \rightarrow y^{1} F^{\circ}$ & 0.0574 & 0.5367 & 1901 & 5 & 7 & $1.2162(-1)$ & 3.8063 & $1.6029(8)$ \\
\hline$a^{1} D^{\prime} \rightarrow x^{i} F^{o}$ & 0.0574 & 0.5597 & 1814 & 5 & 7 & $5.8569(-2)$ & 1.7489 & $8.4792(7)$ \\
\hline$a^{l} D^{*} \rightarrow v^{l} F^{v}$ & 0.0574 & 0.5723 & 1770 & $s$ & 7 & $3.2987(-2)$ & $9.6098(-1)$ & $5.0177(7)$ \\
\hline$a^{1} D^{*} \rightarrow t^{1} F^{0}$ & 0.0574 & 0.5800 & 1744 & 5 & 7 & $2.0398(-2)$ & $5.8553(-1)$ & $3.1956(7)$ \\
\hline$a^{1} D^{e} \rightarrow r^{1} F^{\circ}$ & 0.0574 & 0.5849 & 1727 & 5 & 7 & $1.3441(-2)$ & $3.8218(-1)$ & $2.1459(7)$ \\
\hline$b^{1} D^{0} \rightarrow y^{1} p^{0}$ & 0.4574 & 0.4865 & 31270 & 5 & 3 & $5.2556(-2)$ & $2.7054(1)$ & $5.9743(5)$ \\
\hline$b^{1} D^{4} \rightarrow x^{1} P^{\circ}$ & 0.4574 & 0.5000 & 21360 & 5 & 3 & $2.4933(-1)$ & $8.7668(1)$ & $6.0743(6)$ \\
\hline$b^{1} D^{*} \rightarrow w^{1} p^{\circ}$ & 0.4574 & 0.5358 & 11612 & 5 & 3 & $1.2924(-3)$ & $2.4701(-1)$ & $1.0656(5)$ \\
\hline$b^{1} D^{e} \rightarrow v^{1} P^{0}$ & 0.4574 & 0.5434 & 10585 & 5 & 3 & $1.6896(-2)$ & 2.9440 & $1.6764(6)$ \\
\hline$b^{1} D^{*} \rightarrow u^{2} P^{0}$ & 0.4574 & 0.5587 & 8996 & 5 & 3 & $6.8440(-4)$ & $1.0134(-1)$ & $9.4019(4)$ \\
\hline$b^{1} D^{*} \rightarrow t^{1} p^{0}$ & 0.4574 & 0.5639 & 8553 & 5 & 3 & $5.1314(-3)$ & $7.2239(-1)$ & $7.7988(5)$ \\
\hline$b^{1} D^{\circ} \rightarrow s^{1} P^{\circ}$ & 0.4574 & 0.5711 & 8015 & 5 & 3 & $3.5544(-4)$ & $4.6892(-2)$ & $6.1514(4)$ \\
\hline$b^{1} D^{0} \rightarrow r^{1} P^{0}$ & 0.4574 & 0.5753 & 7727 & 5 & 3 & $2.4093(-3)$ & $3.0645(-1)$ & $4.4857(5)$ \\
\hline$b^{1} D^{0} \rightarrow a^{1} p^{0}$ & 0.4574 & 0.5785 & 7520 & 5 & 3 & $2.0549(-4)$ & $2.5436(-2)$ & $4.0396(4)$ \\
\hline$b^{1} D^{C} \rightarrow p^{1} P^{\circ}$ & 0.4574 & 0.5822 & 7302 & 5 & 3 & $1.3661(-3)$ & $1.6419(-1)$ & $2.8483(5)$ \\
\hline$b^{1} D^{e} \rightarrow o^{1} P^{\circ}$ & 0.4574 & 0.5834 & 7230 & 5 & 3 & $1.2987(-4)$ & $1.5455(-2)$ & 2.7618 (4) \\
\hline$b^{1} D^{e} \rightarrow y^{1} D^{\circ}$ & 0.4574 & 0.5149 & 15838 & 5 & 5 & $1.6702(-1)$ & 4.3541 (1) & $4,4417(6)$ \\
\hline$b^{1} D^{a} \rightarrow x^{1} D^{\circ}$ & 0.4574 & 0.5495 & 9890 & 5 & 5 & $8.3161(-3)$ & 1.3538 & 5.6709 \\
\hline$b^{1} D^{*} \rightarrow w^{1} D^{\circ}$ & 0.4574 & 0.5664 & 8356 & 5 & 5 & $1.4645(-3)$ & $2.0145(-1)$ & 1.3989 (5) \\
\hline$b^{1} D^{\circ} \rightarrow v^{1} D^{\circ}$ & 0.4574 & 0.5773 & 7595 & 5 & 5 & $4.4860(-4)$ & $5.6085(-2)$ & $5.1870(4)$ \\
\hline$b^{1} D^{e} \rightarrow u^{1} D^{0}$ & 0.4574 & 0.5831 & 7246 & $s$ & 5 & $1.8250(-4)$ & $2.1766(-2)$ & $2.3187(4)$ \\
\hline$b^{1} D^{0} \rightarrow z^{1} F^{\circ}$ & 0.4574 & 0.4863 & 31517 & 5 & 7 & $4.9481(-1)$ & $2.5673(2)$ & $2.3727(6)$ \\
\hline$b^{1} D^{e} \rightarrow y^{1} F^{\circ}$ & 0.4574 & 0.5367 & 11489 & 5 & 7 & $1.4766(-1)$ & 2.7924 (1) & $5.3302(6)$ \\
\hline$b^{1} D^{0} \rightarrow x^{1} F^{0}$ & 0.4574 & 0.5597 & 8902 & 5 & 7 & $5.6931(-2)$ & 8.3419 & $3.4230(6)$ \\
\hline$b^{1} D^{\circ} \rightarrow v^{1} F^{\circ}$ & 0.4574 & 0.5723 & 7928 & 5 & 7 & $2.7477(-2)$ & 3.5858 & $2.0826(6)$ \\
\hline$b^{1} D^{4} \rightarrow t^{1} F^{0}$ & 0.4574 & 0.5800 & 7434 & 5 & 7 & $1.5515(-2)$ & 1.8984 & $1.3377(6)$ \\
\hline$b^{1} D^{e} \rightarrow r^{1} F^{\circ}$ & 0.4574 & 0.5849 & 7144 & 5 & 7 & $9.6820(-3)$ & $\begin{array}{l}1.0707 \\
1.1385\end{array}$ & 9.0386 (5) \\
\hline$c^{1} D^{4} \rightarrow w^{1} P^{0}$ & 0.5267 & 0.5358 & 99655 & 5 & 3 & $8.2988(-2)$ & $1.3605(2)$ & $9.3013(4)$ \\
\hline$c^{1} D^{*} \rightarrow v^{1} P^{0}$ & 0.5267 & 0.5434 & 54399 & 5 & 3 & $3.5054(-1)$ & $3.1373(2)$ & $1.3182(6)$ \\
\hline$c^{1} D^{*} \rightarrow u^{1} P^{o}$ & 0.5267 & 0.5587 & 28510 & 5 & 3 & $8.9476(-4)$ & $4.1981(-1)$ & $1.2243(4)$ \\
\hline$c^{1} D^{e} \rightarrow t^{1} P^{a}$ & 0.5267 & 0.5639 & 24489 & 5 & 3 & $2.0927(-2)$ & 8.4338 & $3.8810(5)$ \\
\hline$c^{1} D^{*} \rightarrow s^{1} P^{o}$ & 0.5267 & 0.5711 & 20542 & 5 & 3 & $5.0166(-4)$ & $1.6959(-1)$ & $1.3221(4)$ \\
\hline$c^{1} D^{*} \rightarrow r^{1} P^{0}$ & 0.5267 & 0.5753 & 18753 & 5 & 3 & $6.5912(-3)$ & 2.0343 & $2.0841(5)$ \\
\hline
\end{tabular}

Table V(a). (continued) 


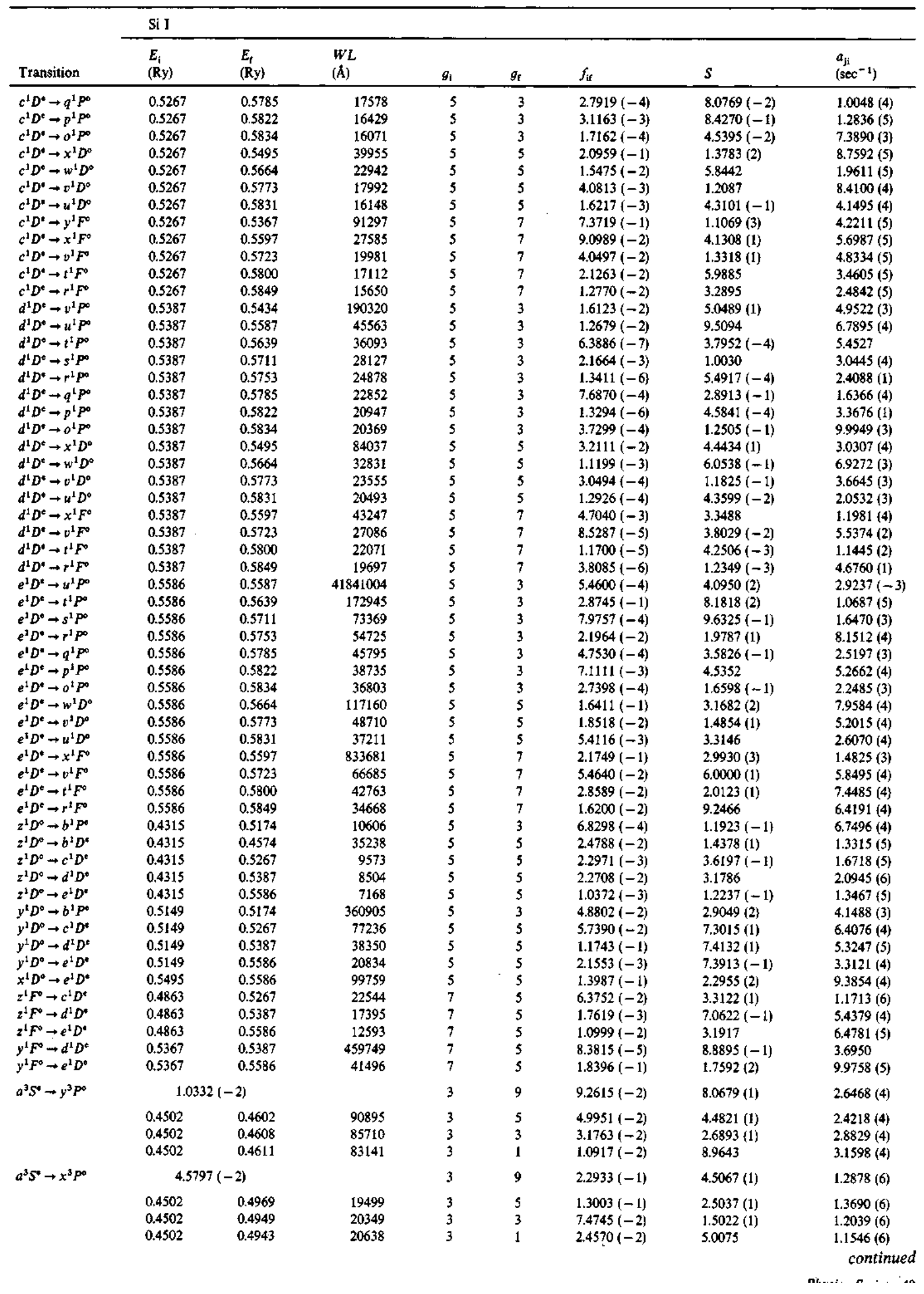

Table V(a). (continued) 


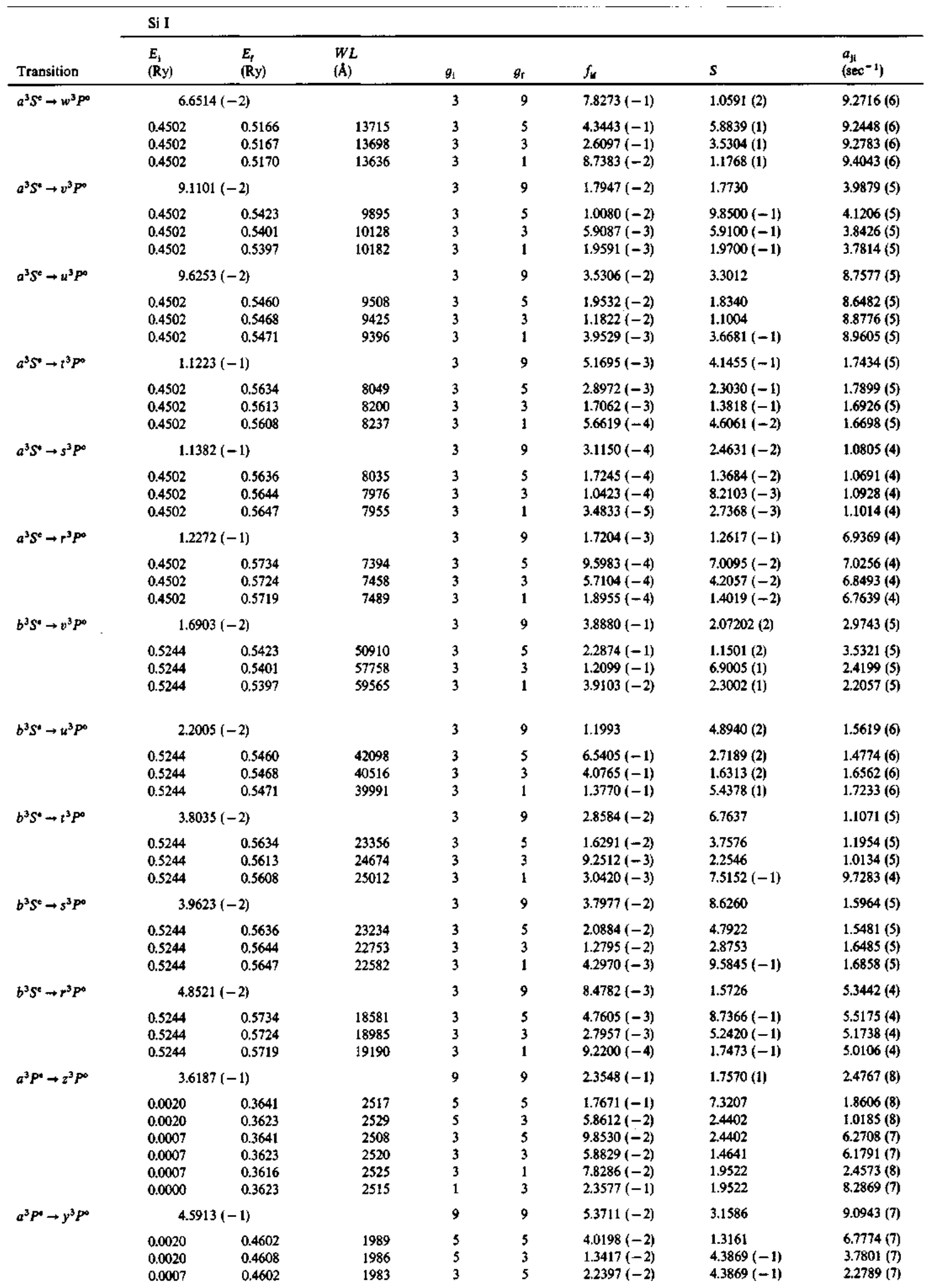

Table V(a). (continued) 


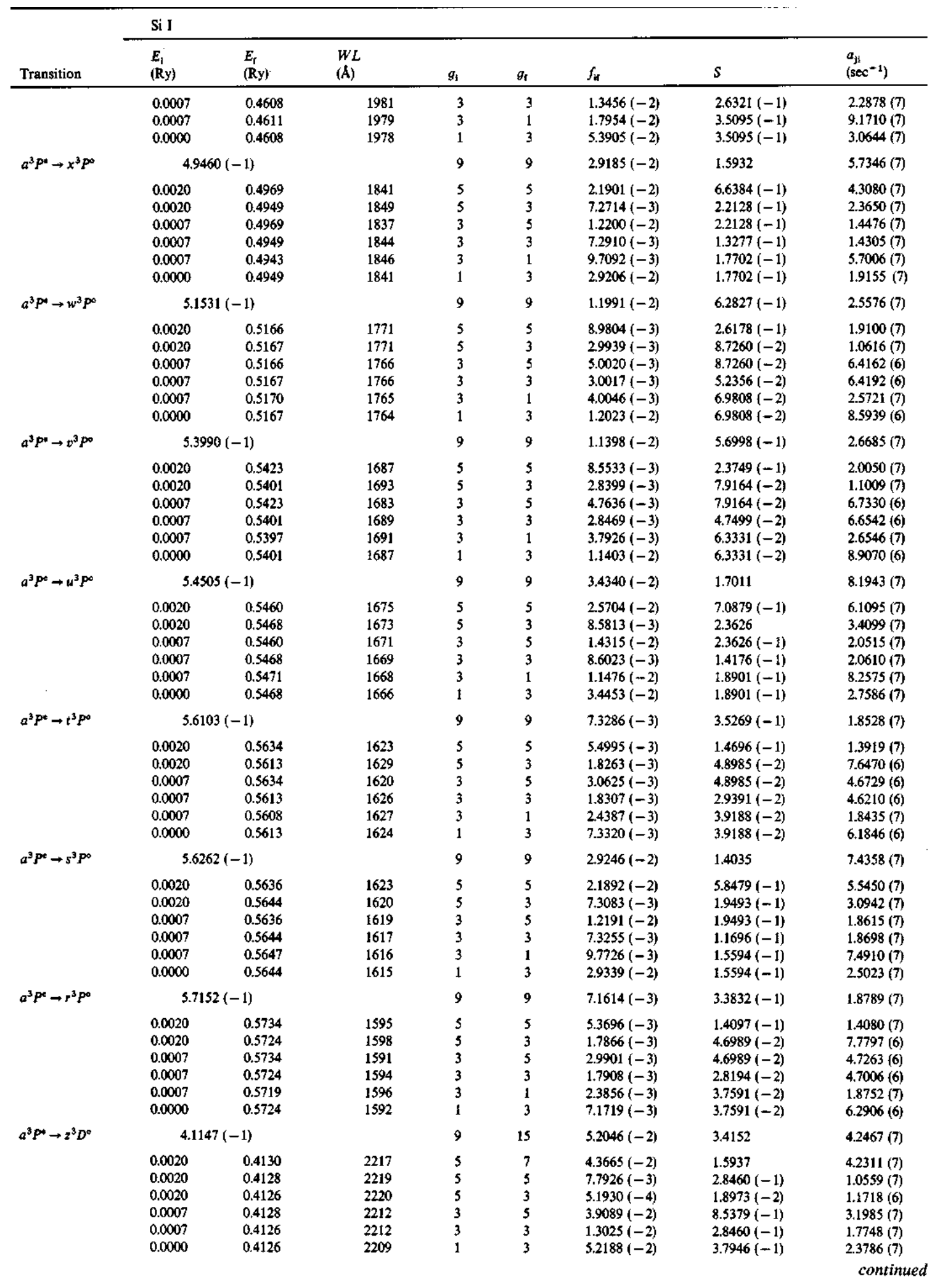

Table V(a). (continued) 


\begin{tabular}{|c|c|c|c|c|c|c|c|c|}
\hline \multirow[b]{2}{*}{ Transition } & \multicolumn{8}{|l|}{ Si I } \\
\hline & $\begin{array}{l}E_{t} \\
\text { (Ry) }\end{array}$ & $\begin{array}{l}E_{f} \\
(\mathrm{Ry})\end{array}$ & $\begin{array}{l}W L \\
(\AA)\end{array}$ & $g_{1}$ & $\theta \mathrm{r}$ & $f_{\text {if }}$ & $s$ & $\begin{array}{l}a_{\mathrm{jh}} \\
\left(\sec ^{-1}\right)\end{array}$ \\
\hline \multirow[t]{7}{*}{$a^{3} P^{e} \rightarrow y^{3} D^{\circ}$} & \multicolumn{2}{|c|}{$4.9278(-1)$} & & 9 & 15 & $2.6931(-1)$ & $1.4756(1)$ & $3.1517(8)$ \\
\hline & 0.0020 & 0.4944 & 1851 & 5 & 7 & $2.2605(-1)$ & 6.8862 & $3.1444(8)$ \\
\hline & 0.0020 & 0.4939 & 1852 & 5 & 5 & $4.0327(-2)$ & 1.2297 & $7.8381(7)$ \\
\hline & 0.0020 & 0.4938 & 1853 & 5 & 3 & $2.6875(-3)$ & $8.1979(-2)$ & 8.6995 (6) \\
\hline & 0.0007 & $0.4939^{\circ}$ & 1847 & 3 & 5 & $2.0218(-1)$ & 3.6890 & 2.3706 (8) \\
\hline & 0.0007 & 0.4938 & 1848 & 3 & 3 & $6.7368(-2)$ & 1.2297 & $1.3155(8)$ \\
\hline & 0.0000 & 0.4938 & 1846 & 1 & 3 & $2.6986(-1)$ & 1.6396 & $1.7616(8)$ \\
\hline \multirow{7}{*}{$a^{3} P^{0} \rightarrow x^{3} D^{0}$} & \multicolumn{2}{|c|}{$5.3699(-1)$} & & 9 & 15 & $1.5518(-1)$ & 7.8026 & $2.1565(8)$ \\
\hline & 0.0020 & 0.5387 & 1698 & 5 & 7 & $1.3028(-1)$ & 3.6412 & $2.1529(8)$ \\
\hline & 0.0020 & 0.5382 & 1700 & 5 & 5 & $2.3240(-2)$ & $6.5021(-1)$ & $5.3654(7)$ \\
\hline & 0.0020 & 0.5379 & 1700 & 5 & 3 & $1.5487(-3)$ & $4.3348(-2)$ & $5.9542(6)$ \\
\hline & 0.0007 & 0.5382 & 1696 & 3 & 5 & $1.1649(-1)$ & 1.9506 & $1.6216(8)$ \\
\hline & 0.0007 & 0.5379 & 1696 & 3 & 3 & $3.8813(-2)$ & $6.5021(-1)$ & $8.9981(7)$ \\
\hline & 0.0000 & 0.5379 & 1694 & 1 & 3 & $1.5546(-1)$ & $8.6695(-1)$ & $1.2045(8)$ \\
\hline \multirow[t]{7}{*}{$a^{3} p^{4} \rightarrow w^{3} D^{\circ}$} & \multicolumn{2}{|c|}{$5.5925(-1)$} & & 9 & 15 & $8.4986(-2)$ & 4.1031 & $1.2810(8)$ \\
\hline & 0.0020 & 0.5611 & 1630 & 5 & 7 & $7.1366(-2)$ & 1.9148 & $1.2798(8)$ \\
\hline & 0.0020 & 0.5605 & 1632 & 5 & 5 & $1.2731(-2)$ & $3.4192(-1)$ & $3.1897(7)$ \\
\hline & 0.0020 & 0.5600 & 1633 & $s$ & 3 & $8.4784(-4)$ & $2.2795(-2)$ & 3.5329 (6) \\
\hline & 0.0007 & 0.5605 & 1628 & 3 & 5 & $6.3807(-2)$ & 1.0258 & $9.6378(7)$ \\
\hline & 0.0007 & 0.5600 & 1629 & 3 & 3 & $2.1247(-2)$ & $3.4192(-1)$ & $5.3374(7)$ \\
\hline & 0.0000 & 0.5600 & 1627 & 1 & 3 & $8.5093(-2)$ & $4.5590(-1)$ & $7.1434(7)$ \\
\hline \multirow[t]{7}{*}{$a^{3} P^{0} \rightarrow v^{3} D^{\circ}$} & \multicolumn{2}{|c|}{$5.7164(-1)$} & & 9 & 15 & $5.0205(-2)$ & 2.3713 & $7.9066(7)$ \\
\hline & 0.0020 & 0.5735 & 1595 & 5 & 7 & $4.2161(-2)$ & 1.1066 & $7.9000(7)$ \\
\hline & 0.0020 & 0.5734 & 1595 & 5 & 5 & $7.5274(-3)$ & $1.9761(-1)$ & $1.9740(7)$ \\
\hline & 0.0020 & 0.5721 & 1599 & 5 & 3 & $5.0062(-4)$ & $1.3174(-2)$ & $2.1776(6)$ \\
\hline & 0.0007 & 0.5734 & 1591 & 3 & 5 & $3.7725(-2)$ & $5.9282(-1)$ & $5.9634(7)$ \\
\hline & 0.0007 & 0.5721 & 1595 & 3 & 3 & $1.2545(-2)$ & $1.9761(-1)$ & $3.2893(7)$ \\
\hline & 0.0000 & 0.5721 & 1593 & 1 & 3 & $5.0241(-2)$ & $2.6348(-1)$ & $4.4019(7)$ \\
\hline \multirow[t]{7}{*}{$a^{3} P^{0} \rightarrow u^{3} D^{0}$} & \multicolumn{2}{|c|}{$5.7908(-1)$} & & 9 & 15 & $3.1695(-2)$ & 1.4778 & $5.1222(7)$ \\
\hline & 0.0020 & 0.5810 & 1574 & 5 & 7 & $2.6620(-2)$ & $6.8964(-1)$ & $5.1199(7)$ \\
\hline & 0.0020 & 0.5809 & 1574 & 5 & 5 & $4.7528(-3)$ & $1.2315(-1)$ & $1.2794(7)$ \\
\hline & 0.0020 & 0.5793 & 1578 & 5 & 3 & $3.1598(-4)$ & $8.2100(-3)$ & $1.4098(6)$ \\
\hline & 0.0007 & 0.5809 & 1571 & 3 & 5 & $2.3819(-2)$ & $3.6945(-1)$ & $3.8647(7)$ \\
\hline & 0.0007 & 0.5793 & 1575 & 3 & 3 & $7.9176(-3)$ & $1.2315(-1)$ & $2.1293(7)$ \\
\hline & 0.0000 & 0.5793 & 1573 & 1 & 3 & $3.1709(-2)$ & $1.6420(-1)$ & $2.8495(7)$ \\
\hline \multirow[t]{7}{*}{$b^{3} P^{4} \rightarrow y^{3} P^{\infty}$} & \multicolumn{2}{|c|}{$1.2806(-2)$} & . & 9 & 9 & $2.4189(-2)$ & $5.1000(1)$ & $3.1862(4)$ \\
\hline & 0.4482 & 0.4602 & 76265 & 5 & 5 & $1.6929(-2)$ & $2.1250(1)$ & $1.9418(4)$ \\
\hline & 0.4482 & 0.4608 & 72581 & $s$ & 3 & $5.9263(-3)$ & 7.0833 & $1.2496(4)$ \\
\hline & 0.4471 & 0.4602 & 69481 & 3 & 5 & $1.0326(-2)$ & 7.0833 & $8.5661(3)$ \\
\hline & 0.4471 & 0.4608 & 66410 & 3 & 3 & $6.4788(-3)$ & 4.2500 & $9.7959(3)$ \\
\hline & 0.4471 & 0.4611 & 64858 & 3 & 1 & $8.8462(-3)$ & 5.6666 & 4.2079 (4) \\
\hline & 0.4468 & 0.4608 & 65015 & i & 3 & $26463(-2)$ & 5.6666 & $1.3907(4)$ \\
\hline$b^{3} P^{*} \rightarrow x^{3} P^{\circ}$ & & $-2)$ & & 9 & 9 & $3.0139(-1)$ & $1.6858(2)$ & $5.6407(6)$ \\
\hline & 0.4482 & 0.4969 & 18728 & 5 & 5 & $2.2786(-1)$ & $7.0240(1)$ & $4.3336(6)$ \\
\hline & 0.4482 & 0.4949 & 19511 & 5 & 3 & $7.2894(-2)$ & $2.3413(1)$ & $2.1282(6)$ \\
\hline & 0.4471 & 0.4969 & 18289 & 3 & 5 & $1.2963(-1)$ & $2.3413(1)$ & $1.5513(6)$ \\
\hline & 0.4471 & 0.4949 & 19036 & 3 & 3 & $7.4720(-2)$ & $1.4048(1)$ & $1.3753(6)$ \\
\hline & 0.4471 & 0.4943 & 19289 & 3 & 1 & $9.8336(-2)$ & $1.8731(1)$ & $5.2902(6)$ \\
\hline & 0.4468 & 0.4949 & 18920 & 1 & 3 & $3.0069(-1)$ & $1.8731(1)$ & $1.8673(6)$ \\
\hline$b^{3} P^{4} \rightarrow w^{3} P^{0}$ & & & & 9 & 9 & $1.9102(-1)$ & $7.4758(1)$ & $7.3023(6)$ \\
\hline & 0.4482 & 0.5166 & 13329 & 5 & 5 & $1.4198(-1)$ & $3.1149(1)$ & $5.3307(6)$ \\
\hline & 0.4482 & 0.5167 & 13313 & 5 & 3 & $4.7381(-2)$ & 1.0383 (1) & $2.9719(6)$ \\
\hline & 0.4471 & 0.5166 & 13106 & 3 & 5 & $8.0226(-2)$ & $1.0383(1)$ & $1.8697(6)$ \\
\hline & 0.4471 & 0.5167 & 13090 & 3 & 3 & $4.8191(-2)$ & 6.2298 & $1.8762(6)$ \\
\hline & 0.4471 & 0.5170 & 13033 & 3 & 1 & $6.4532(-2)$ & 8.3064 & $7.6021(6)$ \\
\hline & 0.4468 & 0.5167 & 13034 & 1 & 3 & $1.9357(-1)$ & 8.3064 & $25329(6)$ \\
\hline$b^{3} P^{e} \rightarrow v^{3} P^{0}$ & & & & 9 & 9 & $2.0654(-2)$ & 5.9595 & $1.4527(6)$ \\
\hline & 0.4482 & 0.5423 & 9692 & 5 & 5 & $1.5564(-2)$ & 2.4831 & 1.1051 (6) \\
\hline & 0.4482 & 0.5401 & 9916 & 5 & 3 & $5.0711(-3)$ & $8.2771(-1)$ & $5.7335(5)$ \\
\hline
\end{tabular}

Table V(a). (continued) 


\begin{tabular}{|c|c|c|c|c|c|c|c|c|}
\hline \multirow[b]{2}{*}{ Transition } & \multicolumn{8}{|l|}{ Si I } \\
\hline & $\begin{array}{l}E_{i} \\
\text { (Ry) }\end{array}$ & $\begin{array}{l}E_{\mathrm{t}} \\
\text { (Ry) }\end{array}$ & $\begin{array}{l}W L \\
(A)\end{array}$ & $B_{1}$ & $\theta_{t}$ & $f_{i f}$ & $S$ & $\begin{array}{l}a_{j l} \\
\text { (sec }^{-1} \text { ) }\end{array}$ \\
\hline & 0.4471 & 0.5423 & 9573 & 3 & 5 & $8.7544(-3)$ & $8.2771(-1)$ & $3.8229(5)$ \\
\hline & 0.4471 & 0.5401 & 9792 & 3 & 3 & $5.1357(-3)$ & $4.9663(-1)$ & $3.5732(5)$ \\
\hline & 0.4471 & 0.5397 & 9842 & 3 & 1 & $6.8122(-3)$ & $6.6217(-1)$ & $1.4073(6)$ \\
\hline & 0.4468 & 0.5401 & 9761 & 1 & 3 & $2.0607(-2)$ & $6.6217(-1)$ & $4.8089(5)$ \\
\hline \multirow[t]{7}{*}{$b^{3} P^{0} \rightarrow u^{3} P^{0}$} & \multicolumn{2}{|c|}{$9.8727(-2)$} & & 9 & 9 & $6.6030(-3)$ & 1.8058 & $5.1695(5)$ \\
\hline & 0.4482 & 0.5460 & 9321 & 5 & 5 & $4.9042(-3)$ & $7.5241(-1)$ & $3.7655(5)$ \\
\hline & 0.4482 & 0.5468 & 9241 & 5 & 3 & $1.6488(-3)$ & $2.5080(-1)$ & $2.1463(5)$ \\
\hline & 0.4471 & 0.5460 & 9211 & 3 & 5 & $2.7572(-3)$ & $2.5080(-1)$ & $1.3008(5)$ \\
\hline & 0.4471 & 0.5468 & 9133 & 3 & 3 & $1.6683(-3)$ & $1.5048(-1)$ & $1.3342(5)$ \\
\hline & 0.4471 & 0.5471 & 9106 & 3 & 1 & $2.2311(-3)$ & $2.0064(-1)$ & 5.3849 (5) \\
\hline & 0.4468 & 0.5468 & 9106 & 1 & 3 & $6.6928(-3)$ & $2.0064(-1)$ & $1.7944(5)$ \\
\hline \multirow[t]{7}{*}{$b^{3} P^{e} \rightarrow t^{3} P^{\circ}$} & \multicolumn{2}{|c|}{$1.1471(-1)$} & & 9 & 9 & $6.3958(-3)$ & 1.5055 & $6.7595(5)$ \\
\hline & 0.4482 & 0.5634 & 7915 & 5 & 5 & $4.8150(-3)$ & $6.2728(-1)$ & $5.1273(5)$ \\
\hline & 0.4482 & 0.5613 & 8060 & 5 & 3 & $1.5759(-3)$ & $2.0909(-1)$ & $2.6962(5)$ \\
\hline & 0.4471 & 0.5634 & 7835 & 3 & 5 & $2.7022(-3)$ & $2.0909(-1)$ & $1.7617(5)$ \\
\hline & 0.4471 & 0.5613 & 7978 & 3 & 3 & $1.5922(-3)$ & $1.2546(-1)$ & $1.6684(5)$ \\
\hline & 0.4471 & 0.5608 & 8013 & 3 & 1 & $2.1136(-3)$ & $1.6727(-1)$ & $6.5865(5)$ \\
\hline & 0.4468 & 0.5613 & 7958 & 1 & 3 & $6.3849(-3)$ & $1.6727(-1)$ & $2.2416(5)$ \\
\hline \multirow[t]{7}{*}{$b^{3} P^{e} \rightarrow s^{3} P^{\infty}$} & \multicolumn{2}{|c|}{$1.1630(-1)$} & & 9 & 9 & $8.8166(-6)$ & $2.0469(-3)$ & $9.5778(2)$ \\
\hline & 0.4482 & 0.5636 & 7901 & 5 & 5 & $6.5581(-6)$ & $8.5289(-4)$ & 7.0077 (2) \\
\hline & 0.4482 & 0.5644 & 7844 & 5 & 3 & $2.2018(-6)$ & $2.8430(-4)$ & $3.9778(2)$ \\
\hline & 0.4471 & 0.5636 & 7821 & 3 & 5 & $3.6804(-6)$ & $2.8430(-4)$ & $2.4077(2)$ \\
\hline & 0.4471 & 0.5644 & 7766 & 3 & 3 & $2.2240(-6)$ & $1.7058(-4)$ & $2.4595(2)$ \\
\hline & 0.4471 & 0.5647 & 7746 & 3 & 1 & $2.9729(-6)$ & $2.2744(-4)$ & $9.9138(2)$ \\
\hline & 0.4468 & 0.5644 & 7747 & 1 & 3 & $8.9178(-6)$ & $2.2744(-4)$ & $3.3038(2)$ \\
\hline \multirow[t]{7}{*}{$b^{3} P^{4} \rightarrow r^{3} P^{0}$} & \multicolumn{2}{|c|}{$1.2519(-1)$} & & 9 & 9 & $3.3771(-3)$ & $7.2833(-1)$ & $4.2515(5)$ \\
\hline & 0.4482 & 0.5734 & 7281 & 5 & $s$ & $2.5322(-3)$ & $3.0347(-1)$ & $3.1861(5)$ \\
\hline & 0.4482 & 0.5724 & 7342 & 5 & 3 & $8.3704(-4)$ & $1.0116(-1)$ & $1.7263(5)$ \\
\hline & 0.4471 & 0.5734 & 7213 & 3 & 5 & $1.4199(-3)$ & $1.0116(-1)$ & $1.0921(5)$ \\
\hline & 0.4471 & 0.5724 & 7274 & 3 & 3 & $8.4493(-4)$ & $6.0694(-2)$ & $1.0653(5)$ \\
\hline & 0.4471 & 0.5719 & 7303 & 3 & 1 & $1.1220(-3)$ & $8.0926(-2)$ & $4.2095(5)$ \\
\hline & 0.4468 & 0.5724 & 7257 & 1 & 3 & $3.3875(-3)$ & $8.0926(-2)$ & $1.4303(5)$ \\
\hline \multirow[t]{7}{*}{$b^{3} P^{e} \rightarrow y^{3} D^{\circ}$} & \multicolumn{2}{|c|}{$4.6452(-2)$} & & 9 & 15 & $6.1132(-1)$ & $3.5533(2)$ & $6.3571(6)$ \\
\hline & 0.4482 & 0.4944 & 19728 & 5 & 7 & $5.1061(-1)$ & $1.6582(2)$ & $6.2502(6)$ \\
\hline & 0.4482 & 0.4939 & 19934 & 5 & 5 & $9.0233(-2)$ & $2.9611(1)$ & $1.5143(6)$ \\
\hline & 0.4482 & 0.4938 & 20013 & 5 & 3 & $5.9919(-3)$ & 1.9740 & $1.6628(5)$ \\
\hline & 0.4471 & 0.4939 & 19438 & 3 & 5 & $4.6271(-1)$ & $8.8832(1)$ & $4.9009(6)$ \\
\hline & 0.4471 & 0.4938 & 19513 & 3 & 3 & $1.5365(-1)$ & $2.9611(1)$ & $2.6915(6)$ \\
\hline & 0.4468 & 0.4938 & 19391 & 1 & 3 & $6.1840(-1)$ & $3.9481(1)$ & 3.6559 (6) \\
\hline \multirow[t]{7}{*}{$b^{3} P^{e} \rightarrow x^{3} D^{\circ}$} & \multicolumn{2}{|c|}{$9.0662(-2)$} & & 9 & 15 & $3.7894(-2)$ & $1.1285(1)$ & $1.5011(6)$ \\
\hline & 0.4482 & 0.5387 & 10071 & 5 & 7 & $3.1767(-2)$ & 5.2664 & $1.4921(6)$ \\
\hline & 0.4482 & 0.5382 & 10134 & 5 & 5 & $5.6376(-3)$ & $9.4044(-1)$ & $3.6614(5)$ \\
\hline & 0.4482 & 0.5379 & 10159 & 5 & 3 & $3.7492(-4)$ & $6.2696(-2)$ & $4.0384(4)$ \\
\hline & 0.4471 & 0.5382 & 10004 & 3 & 5 & $2.8555(-2)$ & 2.8213 & $1.1418(6)$ \\
\hline & 0.4471 & 0.5379 & 10028 & 3 & 3 & $9.4953(-3)$ & $9.4044(-1)$ & $6.2977(5)$ \\
\hline & 0.4468 & 0.5379 & 9996 & 1 & 3 & $3.8102(-2)$ & 1.2539 & $8.4776(5)$ \\
\hline \multirow[t]{7}{*}{$b^{3} P^{t} \rightarrow w^{3} D^{a}$} & & & & 9 & 15 & $2.7947(-2)$ & 6.6823 & $1.7175(6)$ \\
\hline & 0.4482 & 0.5611 & 8074 & 5 & 7 & $2.3465(-2)$ & 3.1184 & $1.7151(6)$ \\
\hline & 0.4482 & 0.5605 & 8115 & 5 & 5 & $4.1690(-3)$ & $5.5686(-1)$ & $4.2231(5)$ \\
\hline & 0.4482 & 0.5600 & 8157 & 5 & 3 & $2.7647(-4)$ & $3.7124(-2)$ & $4.6188(4)$ \\
\hline & 0.4471 & 0.5605 & 8031 & 3 & 5 & $2.1062(-2)$ & 1.6706 & $1.3069(6)$ \\
\hline & 0.4471 & 0.5600 & 8073 & 3 & 3 & $6.9843(-3)$ & $5.5686(-1)$ & $7.1481(5)$ \\
\hline & 0.4468 & 0.5600 & 8052 & 1 & 3 & $2.8009(-2)$ & $7.4248(-1)$ & $9.6044(5)$ \\
\hline$b^{3} P^{\infty} \rightarrow v^{3} D^{\circ}$ & & & & 9 & 15 & $1.6533(-2)$ & 3.5619 & $1.2513(6)$ \\
\hline & 0.4482 & 0.5735 & 7274 & 5 & 7 & $1.3883(-2)$ & 1.6622 & $1.2501(6)$ \\
\hline & 0.4482 & 0.5734 & 7280 & 5 & 5 & $2.4771(-3)$ & $2.9683(-1)$ & $3.1178(5)$ \\
\hline & 0.4482 & 0.5721 & 7360 & 5 & 3 & $1.6333(-4)$ & $1.9788(-2)$ & 3.3518 (4) \\
\hline & 0.4471 & 0.5734 & 7212 & 3 & 5 & $1.2501(-2)$ & $8.9048(-1)$ & $9.6182(5)$ \\
\hline
\end{tabular}

Table V(a). (continued) 


\begin{tabular}{|c|c|c|c|c|c|c|c|c|}
\hline \multirow[b]{2}{*}{ Transition } & \multicolumn{8}{|l|}{ Si I } \\
\hline & $\begin{array}{l}E_{\mathrm{i}} \\
\text { (Ry) }\end{array}$ & $\begin{array}{l}E_{\mathrm{f}} \\
\text { (Ry) }\end{array}$ & $\begin{array}{l}W L \\
(\AA)\end{array}$ & $g_{1}$ & $a_{t}$ & $f_{\mathrm{if}}$ & $s$ & $\begin{array}{l}a_{j 1} \\
\left(\mathrm{sec}^{-1}\right)\end{array}$ \\
\hline & 0.4471 & 0.5721 & 7292 & 3 & 3 & $4.1219(-3)$ & $2.9683(-1)$ & $5.1715(5)$ \\
\hline & 0.4468 & 0.5721 & 7274 & 1 & 3 & $1.6526(-2)$ & $3.9577(-1)$ & $6.9435(5)$ \\
\hline \multirow[t]{7}{*}{$b^{3} P^{4} \rightarrow u^{3} D^{a}$} & \multicolumn{2}{|c|}{$1.3276(-1)$} & & 9 & 15 & $1.0236(-2)$ & 20818 & $8.6946(5)$ \\
\hline & 0.4482 & 0.5810 & 6863 & 5 & 7 & $8.6005(-3)$ & $9.7152(-1)$ & $8.7009(5)$ \\
\hline & 0.4482 & 0.5809 & 6867 & 5 & 5 & $1.5348(-3)$ & $1.7349(-1)$ & $2.1708(5)$ \\
\hline & 0.4482 & 0.5793 & 6951 & 5 & 3 & $1.0108(-4)$ & $1.1566(-2)$ & $2.3258(4)$ \\
\hline & 0.4471 & 0.5809 & 6807 & 3 & 5 & $7.7415(-3)$ & $5.2046(-1)$ & $6.6862(5)$ \\
\hline & 0.4471 & 0.5793 & 6890 & 3 & 3 & $2.5497(-3)$ & $1.7349(-1)$ & $3.5830(5)$ \\
\hline & 0.4468 & 0.5793 & 6874 & 1 & 3 & $1.0221(-2)$ & $2.3131(-1)$ & $4.8088(5)$ \\
\hline \multirow[t]{7}{*}{$c^{3} p^{4} \rightarrow v^{3} P^{p}$} & \multicolumn{2}{|c|}{$1.8173(-2)$} & & 9 & 9 & $4.1793(-1)$ & $6.2094(2)$ & $1.1086(6)$ \\
\hline & 0.5237 & 0.5423 & 49069 & 5 & 5 & $3.2030(-1)$ & $2.5872(2)$ & $8.8719(5)$ \\
\hline & 0.5237 & 0.5401 & 55400 & 5 & 3 & $9.4578(-2)$ & $8.6241(1)$ & $3.4262(5)$ \\
\hline & 0.5224 & 0.5423 & 45927 & 3 & 5 & $1.9011(-1)$ & $8.6241(1)$ & $3.6065(5)$ \\
\hline & 0.5224 & 0.5401 & 51427 & 3 & 3 & $1.0188(-1)$ & 5.1745 (1) & $2.5695(5)$ \\
\hline & 0.5224 & 0.5397 & 52855 & 3 & 1 & $1.3216(-1)$ & $6.8993(1)$ & $9.4653(5)$ \\
\hline & 0.5221 & 0.5401 & 50571 & 1 & 3 & $4.1442(-1)$ & $6.8993(1)$ & $3.6030(5)$ \\
\hline \multirow[t]{7}{*}{$c^{3} P^{\infty} \rightarrow u^{3} P^{a}$} & \multicolumn{2}{|c|}{$2.3324(-2)$} & & 9 & 9 & $3.1979(-1)$ & 3.7018 & $1.3974(6)$ \\
\hline & 0.5237 & 0.5460 & 40831 & 5 & 5 & $2.2951(-1)$ & $1.5424(2)$ & $9.1841(5)$ \\
\hline & 0.5237 & 0.5468 & 39341 & 5 & 3 & $7.9384(-2)$ & $5.1415(1)$ & $5.7003(5)$ \\
\hline & 0.5224 & 0.5460 & 38632 & 3 & 5 & $1.3476(-1)$ & $5.1415(1)$ & $3.6142(5)$ \\
\hline & 0.5224 & 0.5468 & 37295 & 3 & 3 & $8.3737(-2)$ & 3.0849 (1) & $4.0142(5)$ \\
\hline & 0.5224 & 0.5471 & 36850 & 3 & 1 & $1.1302(-1)$ & $4.1132(1)$ & $1.6656(6)$ \\
\hline & 0.5521 & 0.5468 & 36843 & 1 & 3 & $3.3906(-1)$ & $4.1132(1)$ & $5.5519(5)$ \\
\hline \multirow[t]{7}{*}{$c^{3} P^{c} \rightarrow t^{3} P^{\circ}$} & \multicolumn{2}{|c|}{$3.9304(-2)$} & & 9 & 9 & $2.5380(-2)$ & $1.7435(1)$ & $3.1492(5)$ \\
\hline & 0.5237 & 0.5634 & 22961 & 5 & 3 & $1.9222(-2)$ & 7.2645 & $2.4322(5)$ \\
\hline & 0.5237 & 0.5613 & 24233 & 5 & 3 & $6.0699(-3)$ & 2.4215 & $1.1488(5)$ \\
\hline & 0.5224 & 0.5634 & 22249 & 3 & 5 & $1.1021(-2)$ & 2.4215 & $8.9107(4)$ \\
\hline & 0.5224 & 0.5613 & 23441 & 3 & 3 & $6.2749(-3)$ & 1.4529 & $7.6151(4)$ \\
\hline & 0.5224 & 0.5608 & 23746 & 3 & 1 & $8.2589(-3)$ & 1.9372 & $2.9300(5)$ \\
\hline & 0.5221 & 0.5613 & 23262 & 1 & 3 & $2.5293(-2)$ & 1.9372 & $1.0390(5)$ \\
\hline \multirow[t]{7}{*}{$c^{3} P^{0} \rightarrow s^{3} P^{\infty}$} & \multicolumn{2}{|c|}{$4.0893(-2)$} & & 9 & 9 & $1.2339(-2)$ & 8.1473 & $1.6574(5)$ \\
\hline & 0.5237 & 0.5636 & 22843 & 5 & 5 & $9.0276(-3)$ & 3.3947 & $1.1538(5)$ \\
\hline & 0.5237 & 0.5644 & 22378 & 5 & 3 & $3.0718(-3)$ & 1.1316 & $6.8166(4)$ \\
\hline & 0.5224 & 0.5636 & 22138 & 3 & 5 & $5.1750(-3)$ & 1.1316 & $4.2252(4)$ \\
\hline & 0.5224 & 0.5644 & 21701 & 3 & 3 & $3.1676(-3)$ & $6.7894(-1)$ & $4.4860(4)$ \\
\hline & 0.5224 & 0.5647 & 21545 & 3 & 1 & $4.2537(-3)$ & $9.0525(-1)$ & $1.8332(5)$ \\
\hline & 0.5221 & 0.5644 & 21547 & 1 & 3 & $1.2761(-2)$ & $9.0525(-1)$ & $6.1105(4)$ \\
\hline \multirow[t]{7}{*}{$c^{3} p^{4} \rightarrow r^{3} p^{0}$} & \multicolumn{2}{|c|}{$4.9791(-2)$} & & 9 & 9 & $7.5471(-3)$ & 4.0925 & $1.5028(5)$ \\
\hline & 0.5237 & 0.5734 & 18330 & 5 & 5 & $5.6511(-3)$ & 1.7052 & $1.1217(5)$ \\
\hline & 0.5237 & 0.5724 & 18724 & 5 & 3 & $1.8443(-3)$ & $5.6841(-1)$ & $5.8484(4)$ \\
\hline & 0.5224 & 0.5734 & 17873 & 3 & 5 & $3.2197(-3)$ & $5.6841(-1)$ & $4.0328(4)$ \\
\hline & 0.5224 & 0.5724 & 18248 & 3 & 3 & $1.8924(-3)$ & $3.4104(-1)$ & $3.7910(4)$ \\
\hline & 0.5224 & 0.5719 & 18436 & 3 & 1 & $2.4975(-3)$ & $4.5473(-1)$ & $1.4704(5)$ \\
\hline & 0.5221 & 0.5724 & 18139 & 1 & 3 & $7.6152(-3)$ & $4.5473(-1)$ & $5.1463(4)$ \\
\hline \multirow[t]{7}{*}{$c^{3} P^{e} \rightarrow x^{3} D^{\circ}$} & & & & 9 & 15 & $8.9328(-1)$ & $1.5806(3)$ & $1.0024(6)$ \\
\hline & 0.5237 & 0.5387 & 60614 & 5 & 7 & $7.3908(-1)$ & $7.3761(2)$ & $9.5790(5)$ \\
\hline & 0.5237 & 0.5382 & 62962 & 5 & 5 & $1.2706(-1)$ & $1.3172(2)$ & $2.1369(5)$ \\
\hline & 0.5237 & 0.5379 & 63941 & 5 & 3 & $8.3420(-3)$ & 8.7810 & $2.2677(4)$ \\
\hline & 0.5224 & 0.5382 & 57880 & 3 & 5 & $6.9107(-1)$ & $3.9515(2)$ & $8.2512(5)$ \\
\hline & 0.5224 & 0.5379 & 58706 & 3 & 3 & $2.2714(-1)$ & $1.3172(2)$ & $4.3945(5)$ \\
\hline & 0.5221 & 0.5379 & 57593 & 1 & 3 & $9.2611(-1)$ & $1.7562(2)$ & $6.2057(5)$ \\
\hline$c^{3} P^{\circ} \rightarrow w^{3} D^{\circ}$ & & & & 9 & 15 & $1.7303(-2)$ & $1.2452(1)$ & $1.1739(5)$ \\
\hline & 0.5237 & 0.5611 & 24351 & 5 & 7 & $1.4496(-2)$ & 5.8107 & $1.1646(5)$ \\
\hline & 0.5237 & 0.5605 & 24731 & 5 & 5 & $2.5491(-3)$ & 1.0376 & $2.7804(4)$ \\
\hline & 0.5237 & 0.5600 & 25128 & 5 & 3 & $1.6722(-4)$ & $6.9175(-2)$ & $2.9433(3)$ \\
\hline & 0.5224 & 0.5605 & 23906 & 3 & 5 & $1.3185(-2)$ & 3.1129 & 9.2336 (4) \\
\hline & 0.5224 & 0.5600 & 24277 & 3 & 3 & $4.3269(-3)$ & 1.0376 & $4.8952(4)$ \\
\hline & 0.5221 & 0.5600 & 24085 & 1 & 3 & $1.7446(-2)$ & 1.3835 & $6.6848(4)$ \\
\hline
\end{tabular}

Table V(a). (continued) 


\begin{tabular}{|c|c|c|c|c|c|c|c|c|}
\hline \multirow[b]{2}{*}{ Transition } & \multicolumn{8}{|l|}{ Si I } \\
\hline & $\begin{array}{l}E_{1} \\
\text { (Ry) }\end{array}$ & $\begin{array}{l}E_{f} \\
\text { (Ry) }\end{array}$ & $\begin{array}{l}W L \\
\text { (A) }\end{array}$ & $\theta_{1}$ & $g_{t}$ & $f_{1 f}$ & $s$ & $\begin{array}{l}a_{15} \\
\left(\sec ^{-1}\right)\end{array}$ \\
\hline \multirow[t]{7}{*}{$c^{3} P^{n} \rightarrow v^{3} D^{\circ}$} & \multicolumn{2}{|c|}{$4.9917(-2)$} & & 9 & 15 & $1.5649(-2)$ & 8.4647 & $1.8792(5)$ \\
\hline & 0.5237 & 0.5735 & 18289 & 5 & 7 & $1.3122(-2)$ & 3.9502 & $1.8694(5)$ \\
\hline & 0.5237 & 0.5734 & 18323 & 5 & 5 & $2.3386(-3)$ & $7.0539(-1)$ & 4.6455 (4) \\
\hline & 0.5237 & 0.5721 & 18844 & 5 & 3 & $1.5161(-4)$ & $4.7026(-2)$ & 4.7467 (3) \\
\hline & 0.5224 & 0.5734 & 17867 & 3 & 5 & $1.1992(-2)$ & 2.1162 & $1.5032(5)$ \\
\hline & 0.5224 & 0.5721 & 18361 & 3 & 3 & $3.8898(-3)$ & $7.0539(-1)$ & $7.6958(4)$ \\
\hline & 0.5221 & 0.5721 & 18251 & 1 & 3 & $1.5653(-2)$ & $9.4052(-1)$ & $1.0448(5)$ \\
\hline \multirow[t]{7}{*}{$c^{3} p^{n} \rightarrow u^{3} D^{\infty}$} & \multicolumn{2}{|c|}{$5.7355(-2)$} & & 9 & 15 & $1.0286(-2)$ & 4.8420 & $1.6307(5)$ \\
\hline & 0.5237 & 0.5810 & 15893 & 5 & 7 & $8.6377(-3)$ & 2.2596 & $1.6294(5)$ \\
\hline & 0.5237 & 0.5809 & 15918 & 5 & 5 & $1.5400(-3)$ & $4.0350(-1)$ & $4.0543(4)$ \\
\hline & 0.5237 & 0.5793 & 16374 & 5 & 3 & $9.9799(-5)$ & $2.6900(-2)$ & $4.1376(3)$ \\
\hline & 0.5224 & 0.5809 & 15572 & 3 & 5 & $7.8710(-3)$ & 1.2105 & $1.2990(5)$ \\
\hline & 0.5224 & 0.5793 & 16009 & 3 & 3 & $2.5519(-3)$ & $4.0350(-1)$ & 6.6410 (4) \\
\hline & 0.5221 & 0.5793 & 15925 & 1 & 3 & $1.0261(-2)$ & $5.3800(-1)$ & $8.9954(4)$ \\
\hline \multirow[t]{4}{*}{$z^{3} P^{0} \rightarrow a^{3} S^{4}$} & \multicolumn{2}{|c|}{$8.6933(-2)$} & & 9 & 3 & $1.1777(-1)$ & $3.6577(1)$ & $2.1446(7)$ \\
\hline & 0.3641 & 0.4502 & 10588 & 5 & 3 & $1.1659(-1)$ & $2.0320(1)$ & $1.1559(7)$ \\
\hline & 0.3623 & 0.4502 & 10374 & 3 & 3 & $1.1900(-1)$ & $1.2192(1)$ & $7.3749(6)$ \\
\hline & 0.3616 & 0.4502 & 10292 & 1 & 3 & $1.1994(-1)$ & 4.0641 & $2.5175(6)$ \\
\hline \multirow[t]{4}{*}{$z^{3} p^{0} \rightarrow b^{3} S^{4}$} & \multicolumn{2}{|c|}{$1.6113(-1)$} & & 9 & 3 & $3.3298(-3)$ & $5.5796(-1)$ & $2.0832(6)$ \\
\hline & 0.3641 & 0.5244 & 5686 & 5 & 3 & $3.3118(-3)$ & $3,0998(-1)$ & $1.1387(6)$ \\
\hline & 0.3623 & 0.5244 & 5624 & 3 & 3 & $3.3486(-3)$ & $1.8599(-1)$ & $7.0623(5)$ \\
\hline & 0.3616 & 0.5244 & 5599 & 1 & 3 & $3.3631(-3)$ & $6.1996(-2)$ & $2.3847(5)$ \\
\hline \multirow[t]{7}{*}{$2^{3} P^{0} \rightarrow b^{3} P^{0}$} & \multicolumn{2}{|c|}{$8.4458(-2)$} & & 9 & 9 & $4.0965(-1)$ & $1.3096(2)$ & $2.3471(7)$ \\
\hline & 0.3641 & 0.4482 & 10830 & 5 & 5 & $3.0608(-1)$ & $5.4566(1)$ & $1.7405(7)$ \\
\hline & 0.3641 & 0.4471 & 10982 & 5 & 3 & $1.0061(-1)$ & 1.8189 (1) & $9.2717(6)$ \\
\hline & 0.3623 & 0.4482 & 10606 & 3 & 5 & $1.7364(-1)$ & 1.8189 (1) & $6.1778(6)$ \\
\hline & 0.3623 & 0.4471 & 10752 & 3 & 3 & $1.0277(-1)$ & $1.0913(1)$ & $5.9288(6)$ \\
\hline & 0.3623 & 0.4468 & 10790 & 3 & 1 & $1.3655(-1)$ & 1.4551 (1) & $2.3473(7)$ \\
\hline & 0.3616 & 0.4471 & 10664 & 1 & 3 & $4.1446(-1)$ & $1.4551(1)$ & $8.1026(6)$ \\
\hline \multirow[t]{7}{*}{$z^{3} p^{0} \rightarrow c^{3} p^{0}$} & \multicolumn{2}{|c|}{$1.5986(-1)$} & & 9 & 9 & $1.4875(-2)$ & 2.5124 & 3.0534 (6) \\
\hline & 0.3641 & 0.5237 & 5710 & 5 & 5 & $1.1138(-2)$ & 1.0468 & $2.2785(6)$ \\
\hline & 0.3641 & 0.5224 & 5756 & 5 & 3 & $3.6830(-3)$ & $3.4895(-1)$ & $1.2358(6)$ \\
\hline & 0.3623 & 0.5237 & 5647 & 3 & 5 & $6.2566(-3)$ & $3.4895(-1)$ & 7.8518 (5) \\
\hline & 0.3623 & 0.5224 & 5692 & 3 & 3 & $3.7244(-3)$ & $2.0937(-1)$ & $7.6679(5)$ \\
\hline & 0.3623 & 0.5221 & 5703 & 3 & 1 & $4.9566(-3)$ & $2.7916(-1)$ & $3.0500(6)$ \\
\hline & 0.3616 & 0.5224 & 5667 & $i$ & 3 & $1.4963(-2)$ & $2.7916(-1)$ & $1.0359(6)$ \\
\hline \multirow[t]{7}{*}{$z^{3} P^{0} \rightarrow a^{3} D^{8}$} & & & & 9 & 15 & $5.9962(-1)$ & $2.1401(2)$ & $1.6538(7)$ \\
\hline & 0.3641 & 0.4398 & 12035 & 5 & 7 & $5.0416(-1)$ & $9.9872(1)$ & $1.6584(7)$ \\
\hline & 0.3641 & 0.4383 & 12274 & 5 & 5 & $8.8268(-2)$ & 1.7834 (1) & $3.9077(6)$ \\
\hline & 0.3641 & 0.4376 & 12399 & 5 & 3 & $5.8251(-3)$ & 1.1890 & $4.2116(5)$ \\
\hline & 0.3623 & 0.4383 & 11987 & 3 & 5 & $4.5192(-1)$ & $5.3503(1)$ & $1.2587(7)$ \\
\hline & 0.3623 & 0.4376 & 12107 & 3 & 3 & $1.4915(-1)$ & 1.7834 (1) & $6.7876(6)$ \\
\hline & 0.3616 & 0.4376 & 11995 & 1 & 3 & $6.0217(-1)$ & $2.3779(1)$ & 9.3050 (6) \\
\hline$z^{3} P^{\infty} \rightarrow b^{3} D^{\varphi}$ & & & & 9 & 15 & $4.2795(-3)$ & $7.3574(-1)$ & $5.0868(5)$ \\
\hline & 0.3641 & 0.5212 & 5799 & 5 & 7 & $3.5967(-3)$ & $3.4335(-1)$ & $5.0948(5)$ \\
\hline & 0.3641 & 0.5196 & 5861 & 5 & 5 & $6.3552(-4)$ & $6.1312(-2)$ & 1.2340 (5) \\
\hline & 0.3641 & 0.5192 & 5874 & 5 & 3 & $4.2270(-5)$ & $4.0875(-3)$ & $1.3616(4)$ \\
\hline & 0.3623 & 0.5196 & 5795 & 3 & 5 & $3.2140(-3)$ & $1.8394(-1)$ & 3.8306 (s) \\
\hline & 0.3623 & 0.5192 & 5808 & 3 & 3 & $1.0689(-3)$ & $6.1312(-2)$ & $2.1135(5)$ \\
\hline & 0.3616 & 0.5192 & 5782 & 1 & 3 & $4.2946(-3)$ & $8.1749(-2)$ & $2.8559(5)$ \\
\hline$y^{3} p^{0} \rightarrow b^{3} S^{e}$ & & & & 9 & 3 & $2.8035(-3)$ & 1.1852 & 2.7555 (5) \\
\hline & & & 14200 & 5 & 3 & $2.8169(-3)$ & $6.5846(-1)$ & $1.5528(5)$ \\
\hline & 0.4608 & 0.5244 & 14336 & 3 & 3 & $2.7905(-3)$ & $3.9508(-1)$ & $9.0580(4)$ \\
\hline & 0.4611 & 0.5244 & 14410 & 1 & 3 & $2.7761(-3)$ & $1.3169(-1)$ & 2.9725 (4) \\
\hline$y^{3} P^{0} \rightarrow c^{3} p^{0}$ & & & & 9 & 9 & $1.5020(-3)$ & $6.4784(-1)$ & $4.7271(4)$ \\
\hline & 0.4602 & 0.5237 & 14350 & 5 & 5 & $1.1427(-3)$ & $2.6993(-1)$ & $3.7011(4)$ \\
\hline & 0.4602 & 0.5224 & 14644 & 5 & 3 & $3.7329(-4)$ & $8.9978(-2)$ & 1.9352 (4) \\
\hline
\end{tabular}

Table V(a). (continued) 


\begin{tabular}{|c|c|c|c|c|c|c|c|c|}
\hline \multirow[b]{2}{*}{ Transition } & \multicolumn{8}{|l|}{$\mathrm{Si} \mathrm{I}$} \\
\hline & $\begin{array}{l}E_{\mathrm{i}} \\
\text { (Ry) }\end{array}$ & $\begin{array}{l}E_{\mathrm{f}} \\
\text { (Ry) }\end{array}$ & $\begin{array}{l}W L \\
(\AA)\end{array}$ & $g_{i}$ & $g_{f}$ & $f_{\mathrm{ir}}$ & $s$ & $\begin{array}{l}a_{\mathrm{H}} \\
\left(\mathrm{sec}^{-1}\right)\end{array}$ \\
\hline \multirow{11}{*}{$y^{3} P^{0} \rightarrow b^{3} D^{6}$} & 0.4608 & 0.5237 & 14489 & 3 & 5 & $6.2885(-4)$ & $8.9978(-2)$ & $1.1990(4)$ \\
\hline & 0.4608 & 0.5224 & 14788 & 3 & 3 & $3.6969(-4)$ & $5.3987(-2)$ & $1.1279(4)$ \\
\hline & 0.4608 & 0.5221 & 14860 & 3 & 1 & $4.9052(-4)$ & $7.1983(-2)$ & 4.4459 (4) \\
\hline & 0.4611 & 0.5224 & 14867 & 1 & 3 & $1.4708(-3)$ & $7.1983(-2)$ & $1.4798(4)$ \\
\hline & \multicolumn{2}{|c|}{$5.9783(-2)$} & & 9 & 15 & $1.3468(-3)$ & $6.0824(-1)$ & $2.3198(4)$ \\
\hline & 0.4602 & 0.5212 & 14929 & 5 & 7 & $1.1551(-3)$ & $2.8385(-1)$ & $2.4691(4)$ \\
\hline & 0.4602 & 0.5196 & 15343 & 5 & 5 & $2.0069(-4)$ & $5.0687(-2)$ & $5.6856(3)$ \\
\hline & 0.4602 & 0.5192 & 15436 & 5 & 3 & $1.3298(-5)$ & $3.3791(-3)$ & $6.2032(2)$ \\
\hline & 0.4608 & 0.5196 & 15501 & 3 & 5 & $9.9329(-4)$ & $1.5206(-1)$ & $1.6545(4)$ \\
\hline & 0.4608 & 0.5192 & 15596 & 3 & 3 & $3.2907(-4)$ & $5.0687(-2)$ & 9.0239 (3) \\
\hline & 0.4611 & 0.5192 & 15684 & 1 & 3 & $1.3088(-3)$ & $6.7582(-2)$ & $1.1829(4)$ \\
\hline \multirow{4}{*}{$x^{3} p^{0} \rightarrow b^{3} s^{5}$} & \multicolumn{2}{|c|}{$2.8401(-2)$} & & 9 & 3 & $1.7871(-1)$ & $1.6990(2)$ & $3.4735(6)$ \\
\hline & 0.4969 & 0.5244 & 33182 & 5 & 3 & $1.7279(-1)$ & $9.4387(1)$ & $1.7442(6)$ \\
\hline & 0.4949 & 0.5244 & 30978 & 3 & 3 & $1.8512(-1)$ & $5.6632(1)$ & $1.2870(6)$ \\
\hline & 0.4943 & 0.5244 & 30332 & 1 & 3 & $1.8903(-1)$ & $1.8877(1)$ & $4.5671(5)$ \\
\hline \multirow[t]{7}{*}{$x^{3} P^{\circ} \rightarrow c^{3} P^{0}$} & \multicolumn{2}{|c|}{$2.7131(-2)$} & & 9 & 9 & $5.5737(-1)$ & $5.5468(2)$ & $3.2955(6)$ \\
\hline & 0.4969 & 0.5237 & 34013 & 5 & 5 & $4.1277(-1)$ & $2.3111(2)$ & $2.3795(6)$ \\
\hline & 0.4969 & 0.5224 & 35707 & 5 & 3 & $1.3107(-1)$ & 7.7038 (1) & $1.1427(6)$ \\
\hline & 0.4949 & 0.5237 & 31702 & 3 & 5 & $2.4609(-1)$ & $7.7038(1)$ & $9.8031(5)$ \\
\hline & 0.4949 & 0.5224 & 33168 & 3 & 3 & $1.4113(-1)$ & 4.6223 (1) & $8.5606(5)$ \\
\hline & 0.4949 & 0.5221 & 33534 & 3 & 1 & $1.8612(-1)$ & $6.1631(1)$ & $3.3133(6)$ \\
\hline & 0.4943 & 0.5224 & 32428 & 1 & 3 & $5.7727(-1)$ & $6.1631(1)$ & $1.2204(6)$ \\
\hline \multirow[t]{7}{*}{$x^{3} P^{\circ} \rightarrow b^{3} D^{\bullet}$} & \multicolumn{2}{|c|}{$2.4318(-2)$} & & 9 & 15 & $8.9256(-1)$ & $9.9099(2)$ & $2.5438(6)$ \\
\hline & 0.4969 & 0.5212 & 37456 & 5 & 7 & $7.5011(-1)$ & $4.6246(2)$ & $2.5475(6)$ \\
\hline & 0.4969 & 0.5196 & 40172 & 5 & $s$ & $1.2486(-1)$ & $8.2582(1)$ & $5.1590(5)$ \\
\hline & 0.4969 & 0.5192 & 40816 & 5 & 3 & $8.1922(-3)$ & 5.5055 & $5.4635(4)$ \\
\hline & 0.4949 & 0.5196 & 36987 & 3 & 5 & $6.7828(-1)$ & $2.4775(2)$ & $1.9846(6)$ \\
\hline & 0.4949 & 0.5192 & 37531 & 3 & 3 & $2.2279(-1)$ & $8.2582(1)$ & $1.0549(6)$ \\
\hline & 0.4943 & 0.5192 & 36587 & 1 & 3 & $9.1391(-1)$ & $1.1011(2)$ & $1.5171(6)$ \\
\hline \multirow[t]{4}{*}{$w^{3} P^{0} \rightarrow b^{3} s^{*}$} & \multicolumn{2}{|c|}{$7.6836(-3)$} & & 9 & 3 & $8.3264(-2)$ & $2.9259(2)$ & $1,1845(5)$ \\
\hline & 0.5166 & 0.5244 & 117507 & 5 & 3 & $8.3983(-2)$ & $1.6255(2)$ & $6.7528(4)$ \\
\hline & 0.5167 & 0.5244 & 118812 & 3 & 3 & $8.3116(-2)$ & $9.7529(1)$ & $3.9275(4)$ \\
\hline & 0.5170 & 0.5244 & 123680 & 1 & 3 & $7.9865(-2)$ & $3.2510(1)$ & $1.1615(4)$ \\
\hline \multirow[t]{7}{*}{$w^{3} p^{\infty} \rightarrow c^{3} p^{*}$} & \multicolumn{2}{|c|}{$6.4141(-3)$} & & 9 & 9 & $4.6789(-2)$ & $1.9696(2)$ & $1.5461(4)$ \\
\hline & 0.5166 & 0.5237 & 128644 & 5 & 5 & $3.8739(-2)$ & $8.2065(1)$ & $1.5596(4)$ \\
\hline & 0.5166 & 0.5224 & 156768 & 5 & 3 & $1.0596(-2)$ & 2.7355 (1) & $4.7881(3)$ \\
\hline & 0.5167 & 0.5237 & 130210 & 3 & 5 & $2.1276(-2)$ & 2.7355 (1) & $5.0243(3)$ \\
\hline & 0.5167 & 0.5224 & 159099 & 3 & 3 & $1.0450(-2)$ & $1.6413(1)$ & 2.7558 \\
\hline & 0.5167 & 0.5221 & 167889 & 3 & 1 & $1.3203(-2)$ & $2.1884(1)$ & $9.3808(3)$ \\
\hline & 0.5170 & 0.5224 & 167952 & 1 & 3 & $3.9610(-2)$ & $2.1884(1)$ & $3.1269(3)$ \\
\hline$w^{3} p^{0} \rightarrow b^{3} D^{4}$ & & & & 9 & 15 & $2.6283(-3)$ & $2.9707(\mathbf{1})$ & $1.6424(2)$ \\
\hline & 0.5166 & 0.5212 & 197190 & 5 & 7 & $2.8325(-3)$ & 9.1965 & $3.4687(2)$ \\
\hline & 0.5166 & 0.5196 & 306191 & 5 & 5 & $3.2516(-4)$ & 1.6422 & $2.3038(1)$ \\
\hline & 0.5166 & 0.5192 & 348004 & 5 & 3 & $1.9050(-5)$ & $1.0948(-1)$ & 1.7372 \\
\hline & 0.5167 & 0.5196 & 315213 & 3 & 5 & $1.5820(-3)$ & 4.9267 & 6.3679 (1) \\
\hline & 0.5167 & 0.5192 & 359704 & 3 & 3 & $4.6165(-4)$ & 1.6422 & $2.3735(1)$ \\
\hline & 0.5170 & 0.5192 & 408370 & 1 & 3 & $1.6276(-3)$ & 2.1896 & $2.1671(1)$ \\
\hline$a^{3} D^{4} \rightarrow y^{3} P^{\circ}$ & & & & 15 & 9 & $1.3569(-3)$ & 2.8248 & $8.4864(3)$ \\
\hline & 0.4398 & 0.4602 & 44732 & 7 & 5 & $1.2787(-3)$ & 1.3182 & $5.9664(3)$ \\
\hline & 0.4383 & 0.4602 & 41710 & 5 & 5 & $3.4290(-4)$ & $2.3540(-1)$ & $1.3149(3)$ \\
\hline & 0.4376 & 0.4602 & 40326 & 3 & 5 & $3.9408(-5)$ & $1.5693(-2)$ & 9.7004 (1) \\
\hline & 0.4383 & 0.4608 & 40583 & 5 & 3 & $1.0570(-3)$ & $7.0620(-1)$ & $7.1314(3)$ \\
\hline & 0.4376 & 0.4608 & 39272 & 3 & 3 & $6.0681(-4)$ & $2.3540(-1)$ & 2.6234 (3) \\
\hline & 0.4376 & 0.4611 & 38724 & 3 & 1 & $8.2059(-4)$ & $3.1387(-1)$ & $1.0948(4)$ \\
\hline$a^{3} D^{e} \rightarrow x^{3} P^{\circ}$ & & & & 15 & 9 & $2.2527(-1)$ & $1.7760(2)$ & $9.8258(6)$ \\
\hline & 0.4398 & 0.4969 & 15964 & 7 & 5 & $2.2527(-1)$ & $8.2879(1)$ & $8.2536(6)$ \\
\hline & 0.4383 & 0.4969 & 15562 & 5 & 5 & $5.7779(-2)$ & $1.4800(1)$ & $1.5915(6)$ \\
\hline & 0.4376 & 0.4969 & 15365 & 3 & 5 & $6.5021(-3)$ & $9.8666(-1)$ & $1.1023(5)$ \\
\hline & 0.4383 & 0.4949 & 16099 & 5 & 3 & $1.6753(-1)$ & $4.4400(1)$ & $7.1849(6)$ \\
\hline
\end{tabular}

Table V(a). (continued) 


\begin{tabular}{|c|c|c|c|c|c|c|c|c|}
\hline \multirow[b]{2}{*}{ Transition } & \multicolumn{8}{|l|}{ Si I } \\
\hline & $\begin{array}{l}E_{i} \\
\text { (Ry) }\end{array}$ & $\begin{array}{l}E_{f} \\
\text { (Ry) }\end{array}$ & $\begin{array}{l}W L \\
\text { (A) }\end{array}$ & $\theta_{1}$ & $g_{\mathrm{r}}$ & $f_{i t}$ & $s$ & $\begin{array}{l}a_{j i} \\
\left(\sec ^{-1}\right)\end{array}$ \\
\hline & 0.4376 & 0.4949 & 15889 & 3 & 3 & $9.4308(-2)$ & $1.4800(1)$ & $2.4914(6)$ \\
\hline & 0.4376 & 0.4943 & 16064 & 3 & 1 & $1.2438(-1)$ & $1.9733(\mathrm{t})$ & $9.6461(6)$ \\
\hline \multirow{7}{*}{$a^{3} D^{\varphi} \rightarrow w^{3} p^{\circ}$} & \multicolumn{2}{|c|}{$7.7797(-2)$} & & 15 & 9 & $6.4778(-3)$ & 3.7469 & $5.2486(5)$ \\
\hline & 0.4398 & 0.5166 & 11867 & 7 & 5 & $6.3939(-3)$ & 1.7486 & $4.2398(5)$ \\
\hline & 0.4383 & 0.5166 & 11643 & 5 & 5 & $1.6293(-3)$ & $3.1224(-1)$ & $8.0173(4)$ \\
\hline & 0.4376 & 0.5166 & 11533 & 3 & 5 & $1.8277(-4)$ & $2.0816(-2)$ & $5.5000(3)$ \\
\hline & 0.4383 & 0.5167 & 11631 & 5 & 3 & $4.8929(-3)$ & $9.3673(-1)$ & $4.0209(5)$ \\
\hline & 0.4376 & 0.5167 & 11521 & 3 & 3 & $2.7443(-3)$ & $3.1224(-1)$ & $1.3792(5)$ \\
\hline & 0.4376 & 0.5170 & 11477 & 3 & 1 & $3.6729(-3)$ & $4.1633(-1)$ & $5.5797(5)$ \\
\hline \multirow[t]{7}{*}{$a^{3} D^{4} \rightarrow v^{3} P^{0}$} & \multicolumn{2}{|c|}{$1.0238(-1)$} & & 15 & 9 & $2.3641(-2)$ & $1.0391(1)$ & $3.3175(6)$ \\
\hline & 0.4398 & 0.5423 & 8895 & 7 & 5 & $2.3653(-2)$ & 4.8489 & $2.7913(6)$ \\
\hline & 0.4383 & 0.5423 & 8769 & 5 & 5 & $5.9988(-3)$ & $8.6588(-1)$ & $5.2035(5)$ \\
\hline & 0.4376 & 0.5423 & 8706 & 3 & 5 & $6.7134(-4)$ & $5.7725(-2)$ & 3.5447 (4) \\
\hline & 0.4383 & 0.5401 & 8952 & 5 & 3 & $1.7629(-2)$ & 2.5976 & $2.4458(6)$ \\
\hline & 0.4376 & 0.5401 & 8886 & 3 & 3 & $9.8662(-3)$ & $8.6588(-1)$ & $8.3341(5)$ \\
\hline & 0.4376 & 0.5397 & 8928 & 3 & 1 & $1.3093(-2)$ & 1.1545 & 3.2870 (6) \\
\hline \multirow[t]{7}{*}{$a^{3} D^{4} \rightarrow u^{3} P^{\infty}$} & \multicolumn{2}{|c|}{$1.0754(-1)$} & & 15 & 9 & $3.3204(-4)$ & $1.3895(-1)$ & $5.1403(4)$ \\
\hline & 0.4398 & 0.5460 & 8581 & 7 & 5 & $3.2789(-4)$ & $6.4843(-2)$ & $4.1577(4)$ \\
\hline & 0.4383 & 0.5460 & 8464 & 5 & 5 & $8.3115(-5)$ & $1.1579(-2)$ & 7.7393 (3) \\
\hline & 0.4376 & 0.5460 & 8405 & 3 & 5 & $9.2993(-6)$ & $7.7194(-4)$ & $5.2681(2)$ \\
\hline & 0.4383 & 0.5468 & 8398 & 5 & 3 & $2.5129(-4)$ & $3.4737(-2)$ & $3.9609(4)$ \\
\hline & 0.4376 & 0.5468 & 8340 & 3 & 3 & $1.4057(-4)$ & $1.1579(-2)$ & $1.3479(4)$ \\
\hline & 0.4376 & 0.5471 & 8318 & 3 & 1 & $1.8794(-4)$ & $1.5439(-2)$ & $5.4361(4)$ \\
\hline \multirow[t]{7}{*}{$a^{3} D^{2} \rightarrow t^{3} P^{0}$} & \multicolumn{2}{|c|}{$1.2352(-1)$} & & 15 & 9 & $7.6282(-3)$ & 2.7792 & $1.5580(6)$ \\
\hline & 0.4398 & 0.5634 & 7375 & 7 & 5 & $7.6310(-3)$ & 1.2969 & $1.3101(6)$ \\
\hline & 0.4383 & 0.5634 & 7288 & 5 & 5 & $1.9306(-3)$ & $2.3160(-1)$ & 2.4245 (5) \\
\hline & 0.4376 & 0.5634 & 7245 & 3 & 5 & $2.1580(-4)$ & $1.5440(-2)$ & $1.6456(4)$ \\
\hline & 0.4383 & 0.5613 & 7412 & 5 & 3 & $5.6950(-3)$ & $6.9479(-1)$ & $1.1525(6)$ \\
\hline & 0.4376 & 0.5613 & 7367 & 3 & 3 & $3.1832(-3)$ & $2.3160(-1)$ & $3.9123(5)$ \\
\hline & 0.4376 & 0.5608 & 7396 & 3 & 1 & $4.2271(-3)$ & $3.0880(-1)$ & $1.5460(6)$ \\
\hline \multirow[t]{7}{*}{$a^{3} D^{*} \rightarrow s^{3} P^{\circ}$} & \multicolumn{2}{|c|}{$1.2510(-1)$} & & 15 & 9 & $4.0779(-6)$ & $1.4558(-3)$ & $8.5442(2)$ \\
\hline & 0.4398 & 0.5636 & 7363 & 7 & 5 & $4.0341(-6)$ & $6.8452(-4)$ & $6.9482(2)$ \\
\hline & 0.4383 & 0.5636 & 7276 & 5 & 5 & $1.0206(-6)$ & $1.2224(-4)$ & $1.2858(2)$ \\
\hline & 0.4376 & 0.5636 & 7233 & 3 & 5 & $1.1408(-7)$ & $8.1491(-6)$ & 8.7269 \\
\hline & 0.4383 & 0.5644 & 7228 & 5 & 3 & $3.0821(-6)$ & $3.6671(-4)$ & 6.5577 (2) \\
\hline & 0.4376 & 0.5644 & 7186 & 3 & 3 & $1.7224(-6)$ & $1.2224(-4)$ & 2.2251 (2) \\
\hline & 0.4376 & 0.5647 & 7168 & 3 & 1 & $2.3020(-6)$ & $1.6298(-4)$ & $8.9638(2)$ \\
\hline \multirow[t]{7}{*}{$a^{3} D^{*} \rightarrow r^{3} p^{a}$} & \multicolumn{2}{|c|}{$1.3400(-1)$} & & 15 & 9 & $3.4411(-3)$ & 1.1556 & $8.2721(5)$ \\
\hline & 0.4398 & 0.5734 & 6822 & 7 & 5 & $3.4303(-3)$ & $5.3927(-1)$ & $6.8830(5)$ \\
\hline & 0.4383 & 0.5734 & 6747 & 5 & 5 & $8.6708(-4)$ & $9.6299(-2)$ & $1.2704(5)$ \\
\hline & 0.4376 & 0.5734 & 6710 & 3 & 5 & $9.6877(-5)$ & $6.4199(-3)$ & $8.6114(3)$ \\
\hline & 0.4383 & 0.5724 & 6800 & 5 & 3 & $2.5812(-3)$ & $28890(-1)$ & $6.2065(5)$ \\
\hline & 0.4376 & 0.5724 & 6762 & 3 & 3 & $1.4420(-3)$ & $9.6299(-2)$ & $2.1038(5)$ \\
\hline & 0.4376 & 0.5719 & 6787 & 3 & 1 & $1.9154(-3)$ & $1.2840(-1)$ & $8.3199(5)$ \\
\hline \multirow[t]{8}{*}{$a^{3} D^{\mathrm{c}} \rightarrow y^{3} D^{\circ}$} & \multicolumn{2}{|c|}{$5.5261(-2)$} & & 15 & 15 & $1.3463(-1)$ & $1.0964(2)$ & $3.3024(6)$ \\
\hline & 0.4398 & 0.4944 & 16685 & 7 & 7 & $1.1822(-1)$ & $4.5462(1)$ & $2.8319(6)$ \\
\hline & 0.4398 & 0.4939 & 16833 & 7 & 5 & $1.4695(-2)$ & 5.7010 & $4.8419(5)$ \\
\hline & 0.4383 & 0.4944 & 16246 & 5 & 7 & $2.1318(-2)$ & 5.7010 & $3.8479(5)$ \\
\hline & 0.4383 & 0.4939 & 16386 & 5 & 5 & $9.4298(-2)$ & $2.5435(1)$ & $2.3423(6)$ \\
\hline & 0.4383 & 0.4938 & 16439 & 5 & 3 & $2.0257(-2)$ & 5.4818 & $8.3320(5)$ \\
\hline & 0.4376 & 0.4939 & 16168 & 3 & 5 & $3.4328(-2)$ & 5,4818 & $5.2551(5)$ \\
\hline & 0.4376 & 0.4938 & 16220 & 3 & 3 & $1.0266(-1)$ & $1.6445(1)$ & $2.6024(6)$ \\
\hline$a^{3} D^{4} \rightarrow x^{3} D^{\circ}$ & 9.9 & & & 15 & 15 & $9.5917(-4)$ & $4.3392(-1)$ & $7.6230(4)$ \\
\hline & 0.4398 & 0.5387 & 9213 & 7 & 7 & $8.4740(-4)$ & $1.7993(-1)$ & $6.6576(4)$ \\
\hline & 0.4398 & 0.5382 & 9266 & 7 & 5 & $1.0566(-4)$ & $2.2564(-2)$ & $1.1491(4)$ \\
\hline & 0.4383 & 0.5387 & 9078 & $s$ & 7 & $1.5100(-4)$ & $2.2564(-2)$ & 8.7292 (3) \\
\hline
\end{tabular}

continued

Table V(a). (continued) 


\begin{tabular}{|c|c|c|c|c|c|c|c|c|}
\hline \multirow[b]{2}{*}{ Transition } & \multicolumn{8}{|l|}{ Si I } \\
\hline & $\begin{array}{l}E_{\mathrm{i}} \\
\text { (Ry) }\end{array}$ & $\begin{array}{l}E_{f} \\
\text { (Ry) }\end{array}$ & $\begin{array}{l}W L \\
(A)\end{array}$ & $g_{i}$ & $g_{\mathrm{f}}$ & $f_{i f}$ & $s$ & $\begin{array}{l}a_{j j} \\
\left(\sec ^{-1}\right)\end{array}$ \\
\hline \multirow{12}{*}{$a^{3} D^{e} \rightarrow w^{3} D^{a}$} & 0.4383 & 0.5382 & 9129 & 5 & 5 & $6.6993(-4)$ & $1.0067(-1)$ & $5.3617(4)$ \\
\hline & 0.4383 & 0.5379 & 9149 & 5 & 3 & $1.4406(-4)$ & $2.1696(-2)$ & $1.9132(4)$ \\
\hline & 0.4376 & 0.5382 & 9061 & 3 & 5 & $2.4244(-4)$ & $2.1696(-2)$ & $1.1818(4)$ \\
\hline & 0.4376 & 0.5379 & 9081 & 3 & 3 & $7.2574(-4)$ & $6.5089(-2)$ & $5.8702(4)$ \\
\hline & \multicolumn{2}{|c|}{$1.2173(-1)$} & & 15 & 15 & $1.9433(-3)$ & $7.1837(-1)$ & $2.3130(5)$ \\
\hline & 0.4398 & 0.5611 & 7513 & 7 & 7 & $1.7205(-3)$ & $2.9788(-1)$ & $2.0330(5)$ \\
\hline & 0.4398 & 0.5605 & 7549 & 7 & 5 & $2.1474(-4)$ & $3.7355(-2)$ & 3.5191 (4) \\
\hline & 0.4383 & 0.5611 & 7423 & 5 & 7 & $3.0574(-4)$ & $3.7355(-2)$ & 2.6439 (4) \\
\hline & 0.4383 & 0.5605 & 7457 & 5 & 5 & $1.3577(-3)$ & $1.6666(-1)$ & $1.6285(5)$ \\
\hline & 0.4383 & 0.5600 & 7493 & 5 & 3 & $2.9120(-4)$ & $3.5918(-2)$ & $5.7653(4)$ \\
\hline & 0.4376 & 0.5605 & 7412 & 3 & 5 & $4.9069(-4)$ & $3.5918(-2)$ & $3.5748(4)$ \\
\hline & 0.4376 & 0.5600 & 7447 & 3 & 3 & $1.4650(-3)$ & $1.0776(-1)$ & $1.7618(5)$ \\
\hline \multirow[t]{8}{*}{$a^{3} D^{\bullet} \rightarrow v^{3} D^{\circ}$} & \multicolumn{2}{|c|}{$1.3413(-1)$} & & 15 & 15 & $1.4345(-3)$ & $4.8128(-1)$ & $2.0729(5)$ \\
\hline & 0.4398 & 0.5735 & 6816 & 7 & 7 & $1.2706(-3)$ & $1.9957(-1)$ & 1.8244 (5) \\
\hline & 0.4398 & 0.5734 & 6821 & 7 & 5 & $1.5922(-4)$ & $2.5027(-2)$ & 3.1957 (4) \\
\hline & 0.4383 & 0.5735 & 6741 & 5 & 7 & $2.2554(-4)$ & $2.5027(-2)$ & 2.3646 (4) \\
\hline & 0.4383 & 0.5734 & 6746 & 5 & 5 & $1.0055(-3)$ & $1.1166(-1)$ & 1.4737 (5) \\
\hline & 0.4383 & 0.5721 & 6815 & 5 & 3 & $2.1451(-4)$ & $2.4064(-2)$ & $5.1340(4)$ \\
\hline & 0.4376 & 0.5734 & 6709 & 3 & 5 & $3.6318(-4)$ & $2.4064(-2)$ & $3.2293(4)$ \\
\hline & 0.4376 & 0.5721 & 6777 & 3 & 3 & $1.0786(-3)$ & $7.2193(-2)$ & $1.5663(5)$ \\
\hline \multirow[t]{8}{*}{$a^{3} D^{*} \rightarrow u^{3} D^{\circ}$} & \multicolumn{2}{|c|}{$1.4157(-1)$} & & 15 & 15 & $9.7515(-4)$ & $3.0997(-1)$ & $1.5697(5)$ \\
\hline & 0.4398 & 0.5810 & 6453 & 7 & 7 & $8.6430(-4)$ & $1.2853(-1)$ & $1.3843(5)$ \\
\hline & 0.4398 & 0.5809 & 6457 & 7 & 5 & $1.0832(-4)$ & $1.6118(-2)$ & 2.4257 (4) \\
\hline & 0.4383 & 0.5810 & 6387 & 5 & 7 & $1.5333(-4)$ & $1.6118(-2)$ & 1.7911 (4) \\
\hline & 0.4383 & 0.5809 & 6391 & 5 & 5 & $6.8365(-4)$ & $7.1913(-2)$ & $1.1166(5)$ \\
\hline & 0.4383 & 0.5793 & 6463 & 5 & 3 & $1.4569(-4)$ & $1.5499(-2)$ & $3.8774(4)$ \\
\hline & 0.4376 & 0.5809 & 6357 & 3 & 5 & $2.4686 i(-4)$ & $1.5499(-2)$ & $2.4447(4)$ \\
\hline & 0.4376 & 0.5793 & 6429 & 3 & 3 & $7.3230(-4)$ & $4.6496(-2)$ & $1.1819(5)$ \\
\hline \multirow[t]{7}{*}{$a^{3} D^{4} \rightarrow z^{3} F^{\circ}$} & \multicolumn{2}{|c|}{$1.6443(-2)$} & & 15 & 21 & $1.3986(-1)$ & $3.8276(2)$ & $2.1696(5)$ \\
\hline & 0.4398 & 0.4561 & 55850 & 7 & 9 & $1.2740(-1)$ & 1.6404 (2) & $2.1173(5)$ \\
\hline & 0.4398 & 0.4550 & 59899 & 7 & 7 & $1.0297(-2)$ & $1.4217(1)$ & 1.9134 (4) \\
\hline & 0.4398 & 0.4543 & 63030 & 7 & 5 & $2.7592(-4)$ & $4.0099(-1)$ & 6.4785 (2) \\
\hline & 0.4383 & 0.4550 & 54601 & 5 & 7 & $1.2614(-1)$ & $\mathrm{f} .1337$ (2) & $2.0160(5)$ \\
\hline & 0.4383 & 0.4543 & 57192 & 5 & 5 & $1.5098(-2)$ & $1.4217(1)$ & 3.0775 (4) \\
\hline & 0.4376 & 0.4543 & 54622 & 3 & 5 & $1.4188(-1)$ & $7.6552(1)$ & $1.9023(5)$ \\
\hline \multirow[t]{7}{*}{$a^{3} D^{4} \rightarrow y^{3} F^{\circ}$} & \multicolumn{2}{|c|}{$8.4999(-2)$} & & 15 & 21 & $4.1249(-1)$ & $2.1838(2)$ & $1.7098(7)$ \\
\hline & 0.4398 & 0.5247 & 10730 & 7 & 9 & $3.7847(-1)$ & $9.3592(1)$ & $1.7051(7)$ \\
\hline & 0.4398 & 0.5235 & 10886 & 7 & 7 & $3.2333(-2)$ & 8.1113 & $1.8199(6)$ \\
\hline & 0.4398 & 0.5228 & 10979 & 7 & 5 & $9.0412(-4)$ & $2.2878(-1)$ & 7.0023 (4) \\
\hline & 0.4383 & 0.5235 & 10697 & 5 & 7 & $3.6736(-1)$ & $6.4683(1)$ & $1.5296(7)$ \\
\hline & 0.4383 & 0.5228 & 10788 & 5 & 5 & $4.5678(-2)$ & 8.1113 & $2.6179(6)$ \\
\hline & 0.4376 & 0.5228 & 10693 & 3 & 5 & $4.1357(-1)$ & $4.3676(1)$ & $1.4475(7)$ \\
\hline \multirow[t]{7}{*}{$a^{3} D^{4} \rightarrow x^{3} F^{\circ}$} & \multicolumn{2}{|c|}{$1.1474(-1)$} & & 15 & 21 & $9.9982(-2)$ & 3.9212 (1) & $7.5519(6)$ \\
\hline & 0.4398 & 0.5545 & 7946 & 7 & 9 & $9.1772(-2)$ & $1.6805(1)$ & $7.5401(6)$ \\
\hline & 0.4398 & 0.5532 & 8038 & 7 & 7 & $7.8627(-3)$ & 1.4564 & 8.1172 (5) \\
\hline & 0.4398 & 0.5526 & 8077 & 7 & 5 & $2.2069(-4)$ & $4.1079(-2)$ & 3.1588 (4) \\
\hline & 0.4383 & 0.5532 & 7935 & 5 & 7 & $8.8926(-2)$ & 1.1614 (1) & $6.7298(6)$ \\
\hline & 0.4383 & 0.5526 & 7972 & 5 & 5 & $1.1098(-2)$ & 1.4564 & $1.1646(6)$ \\
\hline & 0.4376 & 0.5526 & 7921 & 3 & 5 & $1.0025(-1)$ & 7.8424 & $6.3952(6)$ \\
\hline$a^{3} D^{4} \rightarrow v^{3} F^{4}$ & & & & 15 & 21 & $3.9578(-2)$ & 1.3690 (1) & $3.8432(6)$ \\
\hline & 0.4398 & 0.5698 & 7008 & 7 & 9 & $3.6329(-2)$ & 5.8672 & $3.8374(6)$ \\
\hline & 0.4398 & 0.5684 & 7086 & 7 & 7 & $3.1139(-3)$ & $5.0849(-1)$ & 4.1364 (5) \\
\hline & 0.4398 & 0.5682 & 7099 & 7 & 5 & $8.7657(-5)$ & $1.4342(-2)$ & $1.6238(4)$ \\
\hline & 0.4383 & 0.5684 & 7006 & 5 & 7 & $3.5164(-2)$ & 4.0549 & $3.4137(6)$ \\
\hline & 0.4383 & 0.5682 & 7019 & 5 & 5 & $4.4012(-3)$ & $5.0849(-1)$ & $5.9587(5)$ \\
\hline & 0.4376 & 0.5682 & 6978 & 3 & 3 & $3.9726(-2)$ & 2.7380 & $3.2645(6)$ \\
\hline$a^{3} D^{4} \rightarrow t^{3} F^{\circ}$ & & & & 15 & 21 & $2.0179(-2)$ & 6.5467 & $2.2275(6)$ \\
\hline & 0.4398 & 0.5788 & 6557 & 7 & 9 & $1.8567(-2)$ & 2.8057 & $2.2402(6)$ \\
\hline & 0.4398 & 0.5772 & 6633 & 7 & 7 & $1.5907(-3)$ & $2.4316(-1)$ & $2.4115(5)$ \\
\hline & 0.4398 & 0.5760 & 6693 & 7 & 5 & $4.4465(-5)$ & $6.8584(-3)$ & $9.2688(3)$ \\
\hline
\end{tabular}

Table V(a). (continued) 


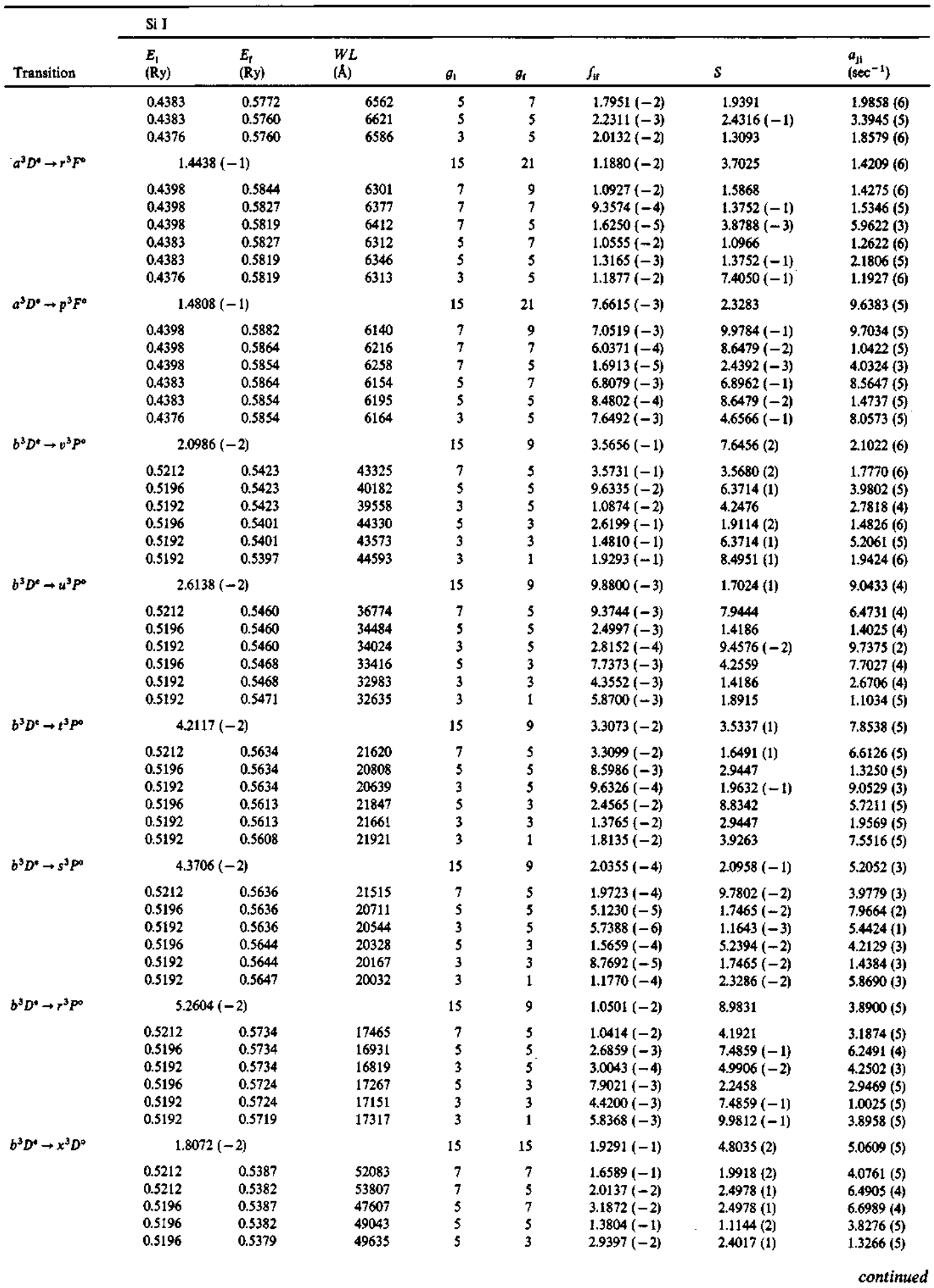

Table V(a). (continued) 


\begin{tabular}{|c|c|c|c|c|c|c|c|c|}
\hline Transition & $\begin{array}{l}E_{\mathrm{i}} \\
\text { (Ry) }\end{array}$ & $\begin{array}{l}E_{\text {q }} \\
\text { (Ry) }\end{array}$ & $\begin{array}{l}W L \\
(\mathcal{A})\end{array}$ & $\theta_{i}$ & $\theta_{r}$ & $f_{\text {iff }}$ & $s$ & $\begin{array}{l}a_{j i} \\
\left(\sec ^{-1}\right)\end{array}$ \\
\hline$b^{3} D^{\circ} \rightarrow w^{3} D^{\circ}$ & & & & 15 & 15 & $4.3104(-5)$ & $4.8092(-2)$ & $5.6320(2)$ \\
\hline$b^{3} D^{e} \rightarrow v^{3} D^{\circ}$ & $\begin{array}{r}5.2 \\
0.5212 \\
0.5212 \\
0.5196 \\
0.5196 \\
0.5196 \\
0.5192 \\
0.5192\end{array}$ & $\begin{array}{l}-2) \\
0.5735 \\
0.5734 \\
0.5735 \\
0.5734 \\
0.5721 \\
0.5734 \\
0.5721\end{array}$ & $\begin{array}{l}17427 \\
17459 \\
16896 \\
16925 \\
17369 \\
16814 \\
17251\end{array}$ & $\begin{array}{r}15 \\
7 \\
7 \\
5 \\
5 \\
5 \\
3 \\
3\end{array}$ & $\begin{array}{r}15 \\
7 \\
5 \\
7 \\
5 \\
3 \\
5 \\
3\end{array}$ & $\begin{array}{l}6.9489(-4) \\
6.1231(-4) \\
7.6638(-5) \\
1.1089(-4) \\
4.9383(-4) \\
1.0372(-4) \\
1.7857(-4) \\
5.2216(-4)\end{array}$ & $\begin{array}{l}5.9302(-1) \\
2.4591(-1) \\
3.0837(-2) \\
3.0837(-2) \\
1.3758(-1) \\
2.9651(-2) \\
2.9651(-2) \\
8.8953(-2)\end{array}$ & $\begin{array}{l}1.5519(4) \\
1.3448(4) \\
2.3474(3) \\
1.8511(3) \\
1.1498(4) \\
3.8227(3) \\
2.5280(3) \\
1.1706(4)\end{array}$ \\
\hline$b^{3} D^{*} \rightarrow u^{3} D^{\circ}$ & $\begin{array}{l}0.5212 \\
0.5212 \\
0.5196 \\
0.5196 \\
0.5196 \\
0.5192 \\
0.5192\end{array}$ & $\begin{array}{l}-2) \\
0.5810 \\
0.5809 \\
0.5810 \\
0.5809 \\
0.5793 \\
0.5809 \\
0.5793\end{array}$ & $\begin{array}{l}15239 \\
15262 \\
14831 \\
14852 \\
15249 \\
14766 \\
15158\end{array}$ & $\begin{array}{r}15 \\
7 \\
7 \\
5 \\
5 \\
5 \\
3 \\
3\end{array}$ & $\begin{array}{r}15 \\
7 \\
5 \\
7 \\
5 \\
3 \\
5 \\
3\end{array}$ & $\begin{array}{l}6.6107(-4) \\
5.8381(-4) \\
7.3101(-5) \\
1.0532(-4) \\
4.6922(-4) \\
9.8487(-5) \\
1.6953(-4) \\
4.9540(-4)\end{array}$ & $\begin{array}{l}4.9441(-1) \\
2.0502(-1) \\
2.5710(-2) \\
2.5710(-2) \\
1.1470(-1) \\
2.4721(-2) \\
2.4721(-2) \\
7.4162(-2)\end{array}$ & $\begin{array}{l}1.9223(4) \\
1.6769(4) \\
2.9308(3) \\
2.2818(3) \\
1.4190(4) \\
4.7085(3) \\
3.1123(3) \\
1.4382(4)\end{array}$ \\
\hline$b^{3} D^{\circ} \rightarrow x^{3} F^{\circ}$ & $\begin{array}{r}3.3 \\
0.5212 \\
0.5212 \\
0.5212 \\
0.5196 \\
0.5196 \\
0.5192\end{array}$ & $\begin{array}{l}-2) \\
0.5545 \\
0.5532 \\
0.5526 \\
0.5532 \\
0.5526 \\
0.5526\end{array}$ & $\begin{array}{l}27390 \\
28511 \\
29007 \\
27115 \\
27564 \\
27269\end{array}$ & $\begin{array}{r}15 \\
7 \\
7 \\
7 \\
5 \\
5 \\
3\end{array}$ & $\begin{array}{r}21 \\
9 \\
7 \\
5 \\
7 \\
5 \\
5\end{array}$ & $\begin{array}{l}4.1985(-1) \\
3.8476(-1) \\
3.2032(-2) \\
8.8793(-4) \\
3.7608(-1) \\
4.6389(-2) \\
4.2084(-1)\end{array}$ & $\begin{array}{l}5.6667(2) \\
2.4286(2) \\
2.1048(1) \\
5.9365(-1) \\
1.6784(2) \\
2.1048(1) \\
1.1333(2)\end{array}$ & $\begin{array}{l}2.6777(6) \\
2.6606(6) \\
2.6281(5) \\
9.8510(3) \\
2.4374(6) \\
4.0725(5) \\
2.2653(6)\end{array}$ \\
\hline$b^{3} D^{4} \rightarrow v^{3} F^{\varphi}$ & $\begin{array}{l}4.8 \\
0.5212 \\
0.5212 \\
0.5212 \\
0.5196 \\
0.5196 \\
0.5192\end{array}$ & $\begin{array}{l}-2) \\
0.5698 \\
0.5684 \\
0.5682 \\
0.5684 \\
0.5682 \\
0.5682\end{array}$ & $\begin{array}{l}18740 \\
19310 \\
19410 \\
18659 \\
18753 \\
18616\end{array}$ & $\begin{array}{r}15 \\
7 \\
7 \\
7 \\
5 \\
5 \\
3\end{array}$ & $\begin{array}{r}21 \\
9 \\
7 \\
5 \\
7 \\
5 \\
5\end{array}$ & $\begin{array}{l}1.1003(-1) \\
1.0089(-1) \\
8.4862(-3) \\
2.3809(-4) \\
9.8054(-2) \\
1.2233(-2) \\
1.1060(-1)\end{array}$ & $\begin{array}{l}1.0167(2) \\
4.3574(1) \\
3.7764 \\
1.0652(-1) \\
3.0115(1) \\
3.7764 \\
2.0335(1)\end{array}$ & $\begin{array}{l}1.4970(6) \\
1.4899(6) \\
1.5179(5) \\
5.8991(3) \\
1.3419(6) \\
2.3199(5) \\
1.2771(6)\end{array}$ \\
\hline$b^{3} D^{4} \rightarrow t^{3} F^{\circ}$ & $\begin{array}{l}0.5212 \\
0.5212 \\
0.5212 \\
0.5196 \\
0.5196 \\
0.5192\end{array}$ & $\begin{array}{l}-2) \\
0.5788 \\
0.5772 \\
0.5760 \\
0.5772 \\
0.5760 \\
0.5760\end{array}$ & $\begin{array}{l}15832 \\
16280 \\
16648 \\
15815 \\
16162 \\
16060\end{array}$ & $\begin{array}{r}15 \\
7 \\
7 \\
7 \\
5 \\
5 \\
3\end{array}$ & $\begin{array}{r}21 \\
9 \\
7 \\
5 \\
7 \\
5 \\
5\end{array}$ & $\begin{array}{l}4.6950(-2) \\
4.3306(-2) \\
3.6495(-3) \\
1.0067(-4) \\
4.1945(-2) \\
5.1477(-3) \\
4.6492(-2)\end{array}$ & $\begin{array}{l}3.6866(1) \\
1.5800(1) \\
1.3693 \\
3.8621(-2) \\
1.0919(1) \\
1.3693 \\
7.3732\end{array}$ & $\begin{array}{l}8.8470(5) \\
8.9636(5) \\
9.1830(4) \\
3.3923(3) \\
7.9898(5) \\
1.3148(5) \\
7.2160(5)\end{array}$ \\
\hline
\end{tabular}

Table V(a). (continued) 


\begin{tabular}{|c|c|c|c|c|c|c|c|c|}
\hline Transition & $\begin{array}{l}E_{\mathrm{i}} \\
(\mathrm{Ry})\end{array}$ & $\begin{array}{l}E_{f} \\
\text { (Ry) }\end{array}$ & $\begin{array}{l}W L \\
(\boldsymbol{A})\end{array}$ & $g_{i}$ & $g_{f}$ & $f_{\text {ir }}$ & $s$ & $\begin{array}{l}a_{j i} \\
\left(\sec ^{-1}\right)\end{array}$ \\
\hline & $\begin{array}{l}0.5196 \\
0.5192\end{array}$ & $\begin{array}{l}0.5819 \\
0.5819\end{array}$ & $\begin{array}{l}14614 \\
14530\end{array}$ & $\begin{array}{l}5 \\
3\end{array}$ & $\begin{array}{l}5 \\
5\end{array}$ & $\begin{array}{l}2.7795(-3) \\
2.5088(-2)\end{array}$ & $\begin{array}{l}6.6857(-1) \\
3.6000\end{array}$ & $\begin{array}{l}8.6818(4) \\
4.7563(5)\end{array}$ \\
\hline$b^{3} D^{e} \rightarrow p^{3} F^{0}$ & \multicolumn{2}{|c|}{$6.6679(-2)$} & & is & 21 & $1.5354(-2)$ & $1.0362(1)$ & $3.9167(5)$ \\
\hline & $\begin{array}{l}0.5212 \\
0.5212 \\
0.5212 \\
0.5196 \\
0.5196 \\
0.5192\end{array}$ & $\begin{array}{l}0.5882 \\
0.5864 \\
0.5854 \\
0.5864 \\
0.5854 \\
0.5854\end{array}$ & $\begin{array}{l}13601 \\
13978 \\
14194 \\
13634 \\
13839 \\
13765\end{array}$ & $\begin{array}{l}7 \\
7 \\
7 \\
5 \\
5 \\
3\end{array}$ & $\begin{array}{l}9 \\
7 \\
5 \\
7 \\
5 \\
5\end{array}$ & $\begin{array}{l}1.4169(-2) \\
1.1948(-3) \\
3.3187(-5) \\
1.3676(-2) \\
1.6896(-3) \\
1.5246(-2)\end{array}$ & $\begin{array}{l}4.4410 \\
3.8488(-1) \\
1.0856(-2) \\
3.0692 \\
3.8488(-1) \\
2.0724\end{array}$ & $\begin{array}{l}3.9735(5) \\
4.0784(4) \\
1.5382(3) \\
3.5055(5) \\
5.8849(4) \\
3.2211(5)\end{array}$ \\
\hline$z^{3} D^{\circ} \rightarrow b^{3} P^{c}$ & \multicolumn{2}{|c|}{$3.4854(-2)$} & & 15 & 9 & $3.2660(-2)$ & $4.2167(1)$ & $5.3115(5)$ \\
\hline & $\begin{array}{l}0.4130 \\
0.4128 \\
0.4126 \\
0.4128 \\
0.4126 \\
0.4126\end{array}$ & $\begin{array}{l}0.4482 \\
0.4482 \\
0.4482 \\
0.4471 \\
0.4471 \\
0.4468\end{array}$ & $\begin{array}{l}25861 \\
25674 \\
25560 \\
26547 \\
26424 \\
26652\end{array}$ & $\begin{array}{l}7 \\
5 \\
3 \\
5 \\
3 \\
3\end{array}$ & $\begin{array}{l}5 \\
5 \\
5 \\
3 \\
3 \\
1\end{array}$ & $\begin{array}{l}3.3021(-2) \\
8.3139(-3) \\
9.2793(-4) \\
2.4119(-2) \\
1.3462(-2) \\
1.7799(-2)\end{array}$ & $\begin{array}{l}1.9678(1) \\
3.5139 \\
2.3426(-1) \\
1.0542(1) \\
3.5139 \\
4.6852\end{array}$ & $\begin{array}{l}4.6114(5) \\
8.4111(4) \\
5.6836(3) \\
3.8032(5) \\
1.2855(5) \\
5.0135(5)\end{array}$ \\
\hline$z^{3} D^{\infty} \rightarrow c^{3} P^{c}$ & \multicolumn{2}{|c|}{$1.1026(-1)$} & & 15 & 9 & $3.1528(-3)$ & 1.2868 & $5.1309(5)$ \\
\hline & $\begin{array}{l}0.4130 \\
0.4128 \\
0.4126 \\
0.4128 \\
0.4126 \\
0.4126\end{array}$ & $\begin{array}{l}0.5237 \\
0.5237 \\
0.5237 \\
0.5224 \\
0.5224 \\
0.5221\end{array}$ & $\begin{array}{l}8233 \\
8214 \\
8202 \\
8309 \\
8297 \\
8320\end{array}$ & $\begin{array}{l}7 \\
5 \\
3 \\
5 \\
3 \\
3\end{array}$ & $\begin{array}{l}5 \\
5 \\
5 \\
3 \\
3 \\
1\end{array}$ & $\begin{array}{l}3.1652(-3) \\
7.9308(-4) \\
8.8247(-5) \\
2.3520(-3) \\
1.3086(-3) \\
1.7400(-3)\end{array}$ & $\begin{array}{l}6.0049(-1) \\
1.0723(-1) \\
7.1487(-3) \\
3.2169(-1) \\
1.0723(-1) \\
1.4297(-1)\end{array}$ & $\begin{array}{l}4.3609(5) \\
7.8402(4) \\
5.2495(3) \\
3.7870(5) \\
1.2679(5) \\
5.0301(5)\end{array}$ \\
\hline$z^{3} D^{\circ} \rightarrow a^{3} D^{\circ}$ & \multicolumn{2}{|c|}{$2.6045(-2)$} & & 15 & 15 & $9.3944(-3)$ & $1.6231(1)$ & $5.1188(4)$ \\
\hline 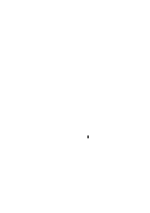 & $\begin{array}{l}0.4130 \\
0.4130 \\
0.4128 \\
0.4128 \\
0.4128 \\
0.4126 \\
0.4126\end{array}$ & $\begin{array}{l}0.4398 \\
0.4383 \\
0.4398 \\
0.4383 \\
0.4376 \\
0.4383 \\
0.4376\end{array}$ & $\begin{array}{l}33985 \\
35965 \\
33663 \\
35604 \\
36678 \\
35384 \\
36445\end{array}$ & $\begin{array}{l}7 \\
7 \\
5 \\
5 \\
5 \\
3 \\
3\end{array}$ & $\begin{array}{l}7 \\
5 \\
7 \\
5 \\
3 \\
5 \\
3\end{array}$ & $\begin{array}{l}8.5958(-3) \\
1.0185(-3) \\
1.5232(-3) \\
6.4242(-3) \\
1.3439(-3) \\
2.3220(-3) \\
6.7630(-3)\end{array}$ & $\begin{array}{l}6.7305 \\
8.4402(-1) \\
8.4402(-1) \\
3.7656 \\
8.1156(-1) \\
8.1156(-1) \\
2.4347\end{array}$ & $\begin{array}{l}4.9664(4) \\
7.3539(3) \\
6.4038(3) \\
3.3790(4) \\
1.1101(4) \\
7.4199(3) \\
3.3951(4)\end{array}$ \\
\hline$z^{3} D^{\circ} \rightarrow b^{3} D^{4}$ & $\begin{array}{l}1.0 \\
0.4130 \\
0.4130 \\
0.4128 \\
0.4128 \\
0.4128 \\
0.4126 \\
0.4126\end{array}$ & $\begin{array}{l}-1) \\
0.5212 \\
0.5196 \\
0.5212 \\
0.5196 \\
0.5192 \\
0.5196 \\
0.5192\end{array}$ & $\begin{array}{l}8420 \\
8550 \\
8400 \\
8530 \\
8558 \\
8517 \\
8545\end{array}$ & $\begin{array}{r}15 \\
7 \\
7 \\
5 \\
5 \\
5 \\
3 \\
3\end{array}$ & $\begin{array}{r}15 \\
7 \\
5 \\
7 \\
5 \\
3 \\
5 \\
3\end{array}$ & $\begin{array}{l}5.2375(-4) \\
4.6879(-4) \\
5.7892(-5) \\
8.2493(-5) \\
3.6245(-4) \\
7.7850(-5) \\
1.3038(-4) \\
3.8984(-4)\end{array}$ & $\begin{array}{l}2.1936(-1) \\
9.0961(-2) \\
1.1407(-2) \\
1.1407(-2) \\
5.0891(-2) \\
1.0968(-2) \\
1.0968(-2) \\
3.2904(-2)\end{array}$ & $\begin{array}{l}4.8565(4) \\
4.4108(4) \\
7.3949(3) \\
5.5696(3) \\
3.3225(4) \\
1.1814(4) \\
7.1928(3) \\
3.5602(4)\end{array}$ \\
\hline$y^{3} D^{\circ} \rightarrow c^{3} P^{e}$ & $\begin{array}{r}2.8 \\
0.4944 \\
0.4939 \\
0.4938 \\
0.4939 \\
0.4938 \\
0.4938\end{array}$ & $\begin{array}{l}-2) \\
0.5237 \\
0.5237 \\
0.5237 \\
0.5224 \\
0.5224 \\
0.5221\end{array}$ & $\begin{array}{l}31146 \\
30645 \\
30460 \\
32013 \\
31811 \\
32148\end{array}$ & $\begin{array}{r}15 \\
7 \\
5 \\
3 \\
5 \\
3 \\
3\end{array}$ & $\begin{array}{l}9 \\
5 \\
5 \\
5 \\
3 \\
3 \\
1\end{array}$ & $\begin{array}{l}9.7966(-2) \\
9.9013(-2) \\
2.5159(-2) \\
2.8124(-3) \\
7.2255(-2) \\
4.0395(-2) \\
5.3296(-2)\end{array}$ & $\begin{array}{l}1.5228(2) \\
7.1062(1) \\
1.2690(1) \\
8.4597(-1) \\
3.8069(1) \\
1.2690(1) \\
1.6919(1)\end{array}$ & $\begin{array}{l}1.0992(6) \\
9.5324(5) \\
1.7874(5) \\
1.2134(4) \\
7.8402(5) \\
2.6633(5) \\
1.0322(6)\end{array}$ \\
\hline$y^{3} D^{\circ} \rightarrow b^{3} D^{4}$ & $\begin{array}{r}2.6 \\
0.4944 \\
0.4944 \\
0.4939 \\
0.4939 \\
0.4939 \\
0.4938 \\
0.4938\end{array}$ & $\begin{array}{l}-2) \\
0.5212 \\
0.5196 \\
0.5212 \\
0.5196 \\
0.5192 \\
0.5196 \\
0.5192\end{array}$ & $\begin{array}{l}34008 \\
36233 \\
33412 \\
35557 \\
36060 \\
35308 \\
35804\end{array}$ & $\begin{array}{r}15 \\
7 \\
7 \\
5 \\
5 \\
5 \\
3 \\
3\end{array}$ & $\begin{array}{r}15 \\
7 \\
5 \\
7 \\
5 \\
3 \\
5 \\
3\end{array}$ & $\begin{array}{l}3.8513(-2) \\
3.5088(-2) \\
4.1293(-3) \\
6.2706(-3) \\
2.6284(-2) \\
5.5851(-3) \\
9.5075(-3) \\
2.8125(-2)\end{array}$ & $\begin{array}{l}6.6306(1) \\
2.7495(1) \\
3.4479 \\
3.4479 \\
1.5383(1) \\
3.3153 \\
3.3153 \\
9.9458\end{array}$ & $\begin{array}{l}2.1134(5) \\
2.0243(5) \\
2.9371(4) \\
2.6773(4) \\
1.3868(5) \\
4.7745(4) \\
3.0523(4) \\
1.4632(5)\end{array}$ \\
\hline$z^{3} F^{\circ} \rightarrow b^{3} D^{\circ}$ & \multicolumn{2}{|c|}{$6.4955(-2)$} & & 21 & 15 & $1.1566(-2)$ & $1.1218(t)$ & $5.4874(5)$ \\
\hline & $\begin{array}{l}0.4561 \\
0.4550 \\
0.4543 \\
0.4550 \\
0.4543 \\
0.4543 \\
\end{array}$ & $\begin{array}{l}0.5212 \\
0.5212 \\
0.5212 \\
0.5196 \\
0.5196 \\
0.5192\end{array}$ & $\begin{array}{l}13999 \\
13766 \\
13611 \\
14117 \\
13953 \\
14030\end{array}$ & $\begin{array}{l}9 \\
7 \\
5 \\
7 \\
5 \\
5\end{array}$ & $\begin{array}{l}7 \\
7 \\
7 \\
5 \\
5 \\
3\end{array}$ & $\begin{array}{l}1.1592(-2) \\
1.3135(-3) \\
5.2461(-5) \\
1.0213(-2) \\
1.8141(-3) \\
9.7146(-3)\end{array}$ & $\begin{array}{l}4.8076 \\
4.1666(-1) \\
1.1752(-2) \\
3.3226 \\
4.1666(-1) \\
2.2435\end{array}$ & $\begin{array}{l}5.0733(5) \\
4.6235(4) \\
1.3495(3) \\
4.7853(5) \\
6.2153(4) \\
5.4861(5)\end{array}$ \\
\hline
\end{tabular}

Table V(b). $f$ - and A-values for S III 


\begin{tabular}{|c|c|c|c|c|c|c|c|c|}
\hline \multirow[b]{2}{*}{ Transition } & \multicolumn{8}{|l|}{ \$ III } \\
\hline & $\begin{array}{l}E_{\mathrm{i}} \\
\text { (Ry) }\end{array}$ & $\begin{array}{l}E_{\mathrm{f}} \\
\text { (Ry) }\end{array}$ & $\begin{array}{l}W L \\
(\boldsymbol{A})\end{array}$ & $g_{1}$ & $\theta_{\mathrm{r}}$ & $f_{\mathrm{ir}}$ & $s$ & $\begin{array}{l}a_{j i} \\
\left(\sec ^{-1}\right)\end{array}$ \\
\hline$a^{1} S^{\prime} \rightarrow z^{1} p^{\infty}$ & 0.2475 & 1.2470 & 912 & 1 & 3 & $2.2255(-3)$ & $6.6798(-3)$ & $5.9524(6)$ \\
\hline$a^{1} S^{*} \rightarrow y^{1} p^{0}$ & 0.2475 & 1.3523 & 825 & 1 & 3 & $6.6156(-2)$ & $1.7964(-1)$ & $2.1620(8)$ \\
\hline$a^{1} S^{\varphi} \rightarrow x^{1} p^{0}$ & 0.2475 & 1.4958 & 730 & 1 & 3 & 2.6474 & 6.3625 & $1.1045(10)$ \\
\hline$a^{1} S^{6} \rightarrow w^{1} P^{\infty}$ & 0.2475 & 1.9258 & 543 & 1 & 3 & $5.5762(-2)$ & $9.9677(-2)$ & $4.2052(8)$ \\
\hline$a^{1} S^{\bullet} \rightarrow v^{\perp} p^{0}$ & r 2475 & 1.9459 & 537 & 1 & 3 & $1.6975(-1)$ & $2.9984(-1)$ & $1.3110(9)$ \\
\hline$b^{+} s^{\circ} \rightarrow w^{1} p^{0}$ & 16654 & 1.9258 & 3500 & 1 & 3 & $2.6945(-1)$ & 3.1042 & $4.8918(7)$ \\
\hline$b^{1} S^{0} \rightarrow v^{1} P^{\circ}$ & 1.6654 & 1.9459 & 3249 & 1 & 3 & 1.0330 & 1.1048 (1) & $2.1761(8)$ \\
\hline$a^{1} p^{*} \rightarrow w^{1} p^{\infty}$ & 1.5269 & 1.9258 & 2284 & 3 & 3 & $1.4281(-1)$ & 3.2222 & $1.8253(8)$ \\
\hline$a^{1} P^{e} \rightarrow v^{1} P^{0}$ & 1.5269 & 1.9459 & 2174 & 3 & 3 & $2.3185(-1)$ & 4.9802 & $3.2695(8)$ \\
\hline$a^{1} P^{*} \rightarrow x^{1} D^{\circ}$ & 1.5269 & 1.8694 & 2660 & 3 & 5 & $7.8965(-1)$ & $2.0750(1)$ & $4.4642(8)$ \\
\hline$z^{1} P^{0} \rightarrow b^{1} S^{*}$ & 1.2470 & 1.6654 & 2178 & 3 & 1 & $7.1949(-2)$ & 1.5477 & $3.0350(8)$ \\
\hline$z^{1} p^{\varphi} \rightarrow a^{1} p^{t}$ & 1.2470 & 1.5269 & 3256 & 3 & 3 & $1.5072(-3)$ & $4.8464(-2)$ & $9.4847(5)$ \\
\hline$z^{1} p^{0} \rightarrow b^{1} D^{e}$ & 1.2470 & 1.6128 & 2491 & 3 & 5 & $6.0640(-2)$ & 1.4920 & $3.9105(7)$ \\
\hline$y^{1} P^{0} \rightarrow b^{1} S^{\circ}$ & 1.3523 & 1.6654 & 2910 & 3 & 1 & $8.0026(-2)$ & 2.3003 & $1.8904(8)$ \\
\hline$y^{1} P^{0} \rightarrow a^{1} P^{*}$ & 1.3523 & 1.5269 & 5221 & 3 & 3 & $2.8700(-1)$ & $1.4794(1)$ & $7.0276(7)$ \\
\hline$y^{1} P^{e} \rightarrow b^{1} D^{\varphi}$ & 1.3523 & 1.6128 & 3498 & 3 & 5 & $5.6460(-1)$ & $1.9506(1)$ & $1.8465(8)$ \\
\hline$x^{1} P^{\circ} \rightarrow b^{1} S^{t}$ & 1.4958 & 1.6654 & $\$ 371$ & 3 & 1 & $7.7858(-2)$ & 4.1316 & $3.3965(7)$ \\
\hline$x^{1} P^{\infty} \rightarrow b^{1} D^{\varphi}$ & 1.4958 & 1.6128 & 7786 & 3 & 5 & $1.1593(-2)$ & $8.9178(-1)$ & $7.6482(5)$ \\
\hline$a^{1} D^{\varphi} \rightarrow z^{1} p^{\infty}$ & 0.1032 & 1.2470 & 797 & 5 & 3 & $3.6960(-1)$ & 4.8469 & 6.4734 (9) \\
\hline$a^{1} D^{*} \rightarrow y^{1} P^{\circ}$ & 0.1032 & 1.3523 & 730 & 5 & 3 & $9.3050(-2)$ & 1.1174 & 1.9436 (9) \\
\hline$a^{1} D^{e} \rightarrow x^{1} P^{\circ}$ & 0.1032 & 1.4958 & 654 & 5 & 3 & $2.3617(-2)$ & $2.5438(-1)$ & $6.1316(8)$ \\
\hline$a^{1} D^{\prime} \rightarrow w^{1} p^{0}$ & 0.1032 & 1.9258 & 500 & 5 & 3 & $2.6074(-2)$ & $2.1459(-1)$ & $1.1596(9)$ \\
\hline$a^{1} D^{*} \rightarrow v^{1} p^{v}$ & 0.1032 & 1.9459 & 495 & 5 & 3 & $6.2091(-4)$ & $5.0543(-3)$ & $2.8225(7)$ \\
\hline$a^{1} D^{\circ} \rightarrow z^{1} D^{\circ}$ & 0.1032 & 0.9492 & 1077 & 5 & 5 & $2.1565(-2)$ & $3.8237(-1)$ & $1.2397(8)$ \\
\hline$a^{1} D^{e} \rightarrow y^{\mathrm{l}} D^{0}$ & 0.1032 & 1.3849 & 711 & 5 & 5 & $9.9367(-1)$ & 1.1629 (1) & $1.3112(10)$ \\
\hline$a^{1} D^{4} \rightarrow x^{1} D^{0}$ & 0.1032 & 1.8694 & 516 & 5 & 3 & $2.4756(-2)$ & $2.1025(-1)$ & $6.2032(8)$ \\
\hline$a^{1} D^{e} \rightarrow z^{1} F^{0}$ & 0.1032 & 1.4362 & 684 & 5 & 7 & 1.3419 & $1.5100(1)$ & $1.3680(10)$ \\
\hline$a^{1} D^{*} \rightarrow y^{l} F^{*}$ & 0.1032 & 1.9241 & 500 & $s$ & 7 & $8.7483(-2)$ & $7.2065(-1)$ & $1.6642(9)$ \\
\hline$b^{1} D^{*} \rightarrow w^{1} P^{0}$ & 1.6128 & 1.9258 & 2912 & $s$ & 3 & $2.1742(-1)$ & 1.0419 (1) & $2.8514(8)$ \\
\hline$b^{1} D^{e} \rightarrow v^{1} P^{0}$ & 1.6128 & 1.9459 & 2735 & 5 & 3 & $9.5199(-3)$ & $4.2870(-1)$ & $1.4141(7)$ \\
\hline$b^{1} D^{*} \rightarrow x^{1} D^{\circ}$ & 1.6128 & 1.8694 & 3551 & 5 & 5 & $1.4237(-1)$ & 8.3223 & $7.5294(7)$ \\
\hline$b^{1} D^{*} \rightarrow y^{1} F^{0}$ & 1.6128 & 1.9241 & 2927 & 5 & 7 & $8.4227(-1)$ & 4.0585 (1) & $4.6830(8)$ \\
\hline$z^{1} D^{0} \rightarrow a^{1} P^{\prime}$ & 0.9492 & 1.5269 & 1577 & 5 & 3 & $7.0623(-2)$ & 1.8336 & 3.1557 (8) \\
\hline$z^{1} D^{0} \rightarrow b^{1} D^{*}$ & 0.9492 & 1.6128 & 1373 & 5 & 5 & $2.6060(-2)$ & $5.8902(-1)$ & $9.2188(7)$ \\
\hline$y^{1} D^{o} \rightarrow a^{1} P^{c}$ & 1.3849 & 1.5269 & 6421 & 5 & 3 & $2.7757(-2)$ & 2.9321 & $7.4928(6)$ \\
\hline$y^{1} D^{\circ} \rightarrow b^{1} D^{4}$ & 1.3849 & 1.6128 & 3999 & 5 & 5 & $1.6998(-2)$ & 1.1188 & $7.0912(6)$ \\
\hline$z^{1} F^{\circ} \rightarrow b^{1} D^{\bullet}$ & 1.4362 & 1.6128 & 5162 & 7 & $s$ & $1.1407(-1)$ & $1.3564(1)$ & $4.0004(7)$ \\
\hline \multirow[t]{4}{*}{$a^{3} S^{6} \rightarrow w^{3} P^{\circ}$} & \multicolumn{2}{|c|}{$3.0948(-1)$} & & 3 & 9 & $9.1937(-1)$ & $2.6736(1)$ & $2.3576(8)$ \\
\hline & 1.5860 & 1.8945 & 2954 & 3 & 5 & $5.0915(-1)$ & 1.4854 (1) & $2.3353(8)$ \\
\hline & 1.5860 & 1.8964 & 2935 & 3 & 3 & $3.0737(-1)$ & 8.9121 & $2.3787(8)$ \\
\hline & 1.5860 & 1.8974 & 2926 & 3 & 1 & $1.0279(-1)$ & 2.9707 & $2.4018(8)$ \\
\hline \multirow[t]{4}{*}{$a^{3} S^{4} \rightarrow v^{3} P^{0}$} & \multicolumn{2}{|c|}{$3.3080(-1)$} & & 3 & 9 & $3.3611(-1)$ & 9.1443 & $9.8478(7)$ \\
\hline & 1.5860 & 1.9200 & 2728 & 3 & 5 & $1.8853(-1)$ & 5.0802 & $1.0136(8)$ \\
\hline & 1.5860 & 1.9130 & 2786 & 3 & 3 & $1.1075(-1)$ & 3.0481 & $9.5119(7)$ \\
\hline & 1.5860 & 1.9116 & 2798 & 3 & 1 & $3.6758(-2)$ & 1.0160 & $9.3902(7)$ \\
\hline \multirow[t]{4}{*}{$2^{3} S^{0} \rightarrow b^{3} P^{4}$} & \multicolumn{2}{|c|}{$3.1830(-1)$} & & 3 & 9 & $9.1985(-5)$ & $2.6009(-3)$ & $2.4952(4)$ \\
\hline & 1.2582 & 1.5783 & 2847 & 3 & 3 & $5.1392(-5)$ & $1.4449(-3)$ & $2.5378(4)$ \\
\hline & 1.2582 & 1.5746 & 2880 & 3 & 3 & $3.0479(-5)$ & $8.6697(-4)$ & 2.4508 (4) \\
\hline & 1.2582 & 1.5731 & 2893 & 3 & 1 & $1.0111(-5)$ & $2.8899(-4)$ & $2.4161(4)$ \\
\hline \multirow[t]{4}{*}{$a^{3} P^{4} \rightarrow z^{3} S^{\circ}$} & \multicolumn{2}{|c|}{1.2530} & & 9 & 3 & $3.4774(-1)$ & 7.4931 & $1.3156(10)$ \\
\hline & 0.0076 & 1.2582 & 729 & 5 & 3 & $3.4707(-1)$ & 4.1628 & $7.2668(9)$ \\
\hline & 0.0027 & 1.2582 & 726 & 3 & 3 & $3.4842 i-1)$ & 2.4977 & $4.4112(9)$ \\
\hline & 0.0000 & 1.2582 & 724 & 1 & 3 & $3.4918(-1)$ & $8.3256(-1)$ & $3.4800(9)$ \\
\hline \multirow[t]{7}{*}{$a^{3} p^{e} \rightarrow z^{3} P^{0}$} & \multicolumn{2}{|c|}{$8.9480(-1)$} & & 9 & 9 & $4.2526(-2)$ & 1.2832 & $2.7349(8)$ \\
\hline & 0.0076 & 0.8998 & 1021 & 5 & 5 & $3.1804(-2)$ & $5.3467(-1)$ & $2.0337(8)$ \\
\hline & 0.0076 & 0.9000 & 1021 & 5 & 3 & $1.0603(-2)$ & $1.7822(-1)$ & $1.1305(8)$ \\
\hline & 0.0027 & 0.8998 & 1016 & 3 & 5 & $1.7765(-2)$ & $1.7822(-1)$ & $6.8906(7)$ \\
\hline & 0.0027 & 0.9000 & 1016 & 3 & 3 & $1.0661(-2)$ & $1.0693(-1)$ & $6.8948(7)$ \\
\hline & 0.0027 & 0.9001 & 1015 & 3 & 1 & $1.4216(-2)$ & $1.4258(-1)$ & $2.7585(8)$ \\
\hline & 0.0000 & 0.9000 & 1012 & 1 & 3 & $4.2774(-2)$ & $1.4258(-1)$ & $9.2769(7)$ \\
\hline
\end{tabular}

Table V(b). (continued) 


\begin{tabular}{|c|c|c|c|c|c|c|c|c|}
\hline \multirow[b]{2}{*}{ Transition } & \multicolumn{8}{|l|}{ S III } \\
\hline & $\begin{array}{l}E_{i} \\
\text { (Ry) }\end{array}$ & $\begin{array}{l}E_{t} \\
(\mathbf{R y})\end{array}$ & $\begin{array}{l}W L \\
\text { (A) }\end{array}$ & $g_{1}$ & $g_{t}$ & $f_{11}$ & $s$ & $\begin{array}{l}a_{\mathrm{ji}} \\
\left(\sec ^{-1}\right)\end{array}$ \\
\hline \multirow[t]{7}{*}{$a^{3} P^{4} \rightarrow y^{3} p^{a}$} & \multicolumn{2}{|c|}{1.2991} & & 9 & 9 & $7.7086(-1)$ & $1.6022(1)$ & $1.0449(10)$ \\
\hline & 0.0076 & 1.3042 & 703 & 5 & 5 & $5.7705(-1)$ & 6.6757 & 7.7923 (9) \\
\hline & 0.0076 & 1.3042 & 703 & 5 & 3 & $1.9235(-1)$ & 2.2252 & $4.3291(9)$ \\
\hline & 0.0027 & 1.3042 & 700 & 3 & 5 & $3.2179(-1)$ & 2.2252 & $2.6268(9)$ \\
\hline & 0.0027 & 1.3042 & 700 & 3 & 3 & $1.9307(-1)$ & 1.3351 & $2.6268(9)$ \\
\hline & 0.0027 & 1.3040 & 700 & 3 & 1 & $2.5739(-1)$ & 1.7802 & $1.0502(10)$ \\
\hline & 0.0000 & 1.3042 & 699 & 1 & 3 & $7.7391(-1)$ & 1.7802 & $3.5245(9)$ \\
\hline \multirow{7}{*}{$a^{3} p^{e} \rightarrow x^{3} p^{e}$} & \multicolumn{2}{|c|}{1.3341} & & 9 & 9 & $8.6062(-2)$ & 1.7418 & $1.2303(9)$ \\
\hline & 0.0076 & 1.3409 & 683 & $s$ & 5 & $6.4509(-2)$ & $7.2574(-1)$ & $9.2113(8)$ \\
\hline & 0.0076 & 1.3372 & 685 & 5 & 3 & $2.1443(-2)$ & $2.4191(-1)$ & $5.0749(8)$ \\
\hline & 0.0027 & 1.3409 & 681 & 3 & 5 & $3.5969(-2)$ & $2.4191(-1)$ & $3.1042(8)$ \\
\hline & 0.0027 & 1.3372 & 683 & 3 & 3 & $2.1522(-2)$ & $1.4515(-1)$ & 3.0785 (8) \\
\hline & 0.0027 & 1.3368 & 683 & 3 & 1 & $2.8687(-2)$ & $1.9353(-1)$ & $1.2303(9)$ \\
\hline & 0.0000 & 1.3372 & 681 & 1 & 3 & $8.6263(-2)$ & $1.9353(-1)$ & $4.1299(8)$ \\
\hline \multirow[t]{7}{*}{$a^{3} P^{*} \rightarrow w^{3} P^{0}$} & & & & 9 & 9 & $2.2834(-2)$ & $3.2615(-1)$ & $6.5538(8)$ \\
\hline & 0.0076 & 1.8945 & 483 & 5 & 5 & $1.7095(-2)$ & $1.3590(-1)$ & $4.8889(8)$ \\
\hline & 0.0076 & 1.8964 & 482 & 5 & 3 & $5.7041(-3)$ & $4.5299(-2)$ & $2.7243(8)$ \\
\hline & 0.0027 & 1.8945 & 482 & 3 & 5 & $9.5218(-3)$ & $4.5299(-2)$ & $1.6423(8)$ \\
\hline & 0.0027 & 1.8964 & 481 & 3 & 3 & $5.7188(-3)$ & $2.7179(-2)$ & $1.6472(8)$ \\
\hline & 0.0027 & 1.8974 & 481 & 3 & 1 & $7.6291(-3)$ & $3.6239(-2)$ & $6.5994(8)$ \\
\hline & 0.0000 & 1.8964 & 481 & 1 & 3 & $2.2908(-2)$ & $3.6239(-2)$ & $2.2058(8)$ \\
\hline$a^{3} P^{0} \rightarrow v^{3} P^{0}$ & & & & 9 & 9 & $2.0040(-2)$ & $2.8305(-1)$ & $5.8822(8)$ \\
\hline & 0.0076 & 1.9200 & 476 & 5 & 5 & $1.5036(-2)$ & $1.1794(-1)$ & $4.4170(8)$ \\
\hline & 0.0076 & 1.9130 & 478 & 5 & 3 & $4.9937(-3)$ & $3.9312(-2)$ & $2.4271(8)$ \\
\hline & 0.0027 & 1.9200 & 475 & 3 & 5 & $8.3746(-3)$ & $3.9312(-2)$ & $1.4836(8)$ \\
\hline & 0.0027 & 1.9130 & 477 & 3 & 3 & $5.0064(-3)$ & $2.3587(-2)$ & $1.4674(8)$ \\
\hline & 0.0027 & 1.9116 & 477 & 3 & 1 & $6.6704(-3)$ & $3.1449(-2)$ & $5.8569(8)$ \\
\hline & 0.0000 & 1.9130 & 476 & 1 & 3 & $2.0054(-2)$ & $3.1449(-2)$ & $1.9650(8)$ \\
\hline$a^{3} p^{c} \rightarrow z^{3} D^{a}$ & & & & 9 & 15 & $2.4566(-2)$ & $8.7164(-1)$ & $6.8552(7)$ \\
\hline & 0.0076 & 0.7664 & 1201 & 5 & 7 & $2.0576(-2)$ & $4.0677(-1)$ & $6.7968(7)$ \\
\hline & 0.0076 & 0.7659 & 1202 & 5 & 5 & $3.6721(-3)$ & $7.2637(-2)$ & $1.6961(7)$ \\
\hline & 0.0076 & 0.7656 & 1202 & 5 & 3 & $2.4472(-4)$ & $4.8425(-3)$ & 1.8826 (6) \\
\hline & 0.0027 & 0.7659 & 1194 & 3 & 5 & $1.8478(-2)$ & $2.1791(-1)$ & $5.1869(7)$ \\
\hline & 0.0027 & 0.7656 & 1194 & 3 & 3 & $6.1574(-3)$ & $7.2637(-2)$ & 2.8786 (7) \\
\hline & 0.0000 & 0.7656 & 1190 & 1 & 3 & $2.4717(-2)$ & $9.6849(-2)$ & $3.8794(7)$ \\
\hline$a^{3} P^{4} \rightarrow y^{3} D^{\circ}$ & & & & 9 & 15 & 1.6373 & $3.2973(1)$ & $1.4184(10)$ \\
\hline & 0.0076 & 1.3464 & 681 & 5 & 7 & 1.3734 & $1.5387(1)$ & $1.4123(10)$ \\
\hline & 0.0076 & 1.3459 & 681 & 5 & 5 & $2.4515(-1)$ & 2.7477 & $3.5268(9)$ \\
\hline & 0.0076 & 1.3446 & 682 & 5 & 3 & $1.6328(-2)$ & $1.8318(-1)$ & $3.9073(8)$ \\
\hline & 0.0027 & 1.3459 & 678 & 3 & 5 & 1.2302 & 8.2431 & $1.0696(10)$ \\
\hline & 0.0027 & 1.3446 & 679 & 3 & 3 & $4.0968(-1)$ & 2.7477 & $5.9252(9)$ \\
\hline & 0.0000 & 1.3446 & 678 & 1 & 3 & 1.6420 & 3.6636 & 7.9485 (9) \\
\hline$a^{3} P^{e} \rightarrow x^{3} D^{0}$ & & & & 9 & 15 & $5.8170(-2)$ & $8.3587(-1)$ & $9.8979(8)$ \\
\hline & 0.0076 & 1.8855 & 485 & 3 & 7 & $4.8835(-2)$ & $3.9007(-1)$ & $9.8807(8)$ \\
\hline & 0.0076 & 1.8833 & 486 & 5 & 5 & $8.7103(-3)$ & $6.9656(-2)$ & $2.4615(8)$ \\
\hline & 0.0076 & 1.8821 & 486 & 5 & 3 & $5.8031(-4)$ & $4.6437(-3)$ & $2.7297(7)$ \\
\hline & 0.0027 & 1.8833 & 485 & 3 & 5 & $4.3664(-2)$ & $2.0897(-1)$ & $7.4422(8)$ \\
\hline & 0.0027 & 1.8821 & 485 & 3 & 3 & $1.4546(-2)$ & $6.9656(-2)$ & $4.1266(8)$ \\
\hline & 0.0000 & 1.8821 & 484 & 1 & 3 & $5.8266(-2)$ & $9.2874(-2)$ & $5.5261(8)$ \\
\hline$b^{3} p^{*} \rightarrow w^{3} p^{a}$ & & & & 9 & 9 & $3.2047(-1)$ & $2.7126(1)$ & $2.6190(8)$ \\
\hline & 1.5783 & 1.8945 & 2882 & 5 & 5 & $2.3826(-1)$ & $1.1303(1)$ & $1.9134(8)$ \\
\hline & 1.5783 & 1.8964 & 2864 & 5 & 3 & $7.9898(-2)$ & 3.7676 & $1.0823(8)$ \\
\hline & 1.5746 & 1.8945 & 2849 & 3 & 5 & $1.3392(-1)$ & 3.7676 & $6.6046(7)$ \\
\hline & 1.5746 & 1.8964 & 2831 & 3 & 3 & $8.0827(-2)$ & 2.2605 & $6.7230(7)$ \\
\hline & 1.5746 & 1.8974 & 2823 & 3 & 1 & $1.0810(-1)$ & 3.0141 & $2.7144(8)$ \\
\hline & 1.5731 & 1.8964 & 2819 & 1 & 3 & $3.2481(-1)$ & 3.0141 & $9.0900(7)$ \\
\hline
\end{tabular}

continued

Table V(b). (continued) 


\begin{tabular}{|c|c|c|c|c|c|c|c|c|}
\hline \multirow[b]{2}{*}{ Transition } & \multicolumn{8}{|l|}{ S III } \\
\hline & $\begin{array}{l}E_{1} \\
\text { (Ry) }\end{array}$ & $\begin{array}{l}E_{f} \\
\text { (Ry) }\end{array}$ & $\begin{array}{l}W L \\
\text { (A) }\end{array}$ & $g_{i}$ & $g_{t}$ & $f_{\mathrm{tr}}$ & $s$ & $\begin{array}{l}a_{j 1} \\
\left(\sec ^{-1}\right)\end{array}$ \\
\hline$b^{3} p^{4} \rightarrow v^{3} p^{\infty}$ & \multicolumn{2}{|c|}{$3.4030(-1)$} & & 9 & 9 & $1.3147(-1)$ & $1.0431(1)$ & $1.2229(8)$ \\
\hline & $\begin{array}{l}1.5783 \\
1.5783 \\
1.5746 \\
1.5746 \\
1.5746 \\
1.5731\end{array}$ & $\begin{array}{l}1.9200 \\
1.9130 \\
1.9200 \\
1.9130 \\
1.9116 \\
1.9130\end{array}$ & $\begin{array}{l}2666 \\
2722 \\
2638 \\
2693 \\
2704 \\
2681 .\end{array}$ & $\begin{array}{l}5 \\
5 \\
3 \\
3 \\
3 \\
1\end{array}$ & $\begin{array}{l}5 \\
3 \\
5 \\
3 \\
1 \\
3\end{array}$ & $\begin{array}{l}9.9004(-2) \\
3.2325(-2) \\
5.5598(-2) \\
3.2683(-2) \\
4.3397(-2) \\
1.3131(-1)\end{array}$ & $\begin{array}{l}4.3461 \\
1.4487 \\
1.4487 \\
8.6922(-1) \\
1.1590 \\
1.1590\end{array}$ & $\begin{array}{l}9.2850(7) \\
4.8478(7) \\
3.1966(7) \\
3.0062(7) \\
1.1876(8) \\
4.0618(7)\end{array}$ \\
\hline$b^{3} P^{*} \rightarrow x^{3} D^{\circ}$ & \multicolumn{2}{|c|}{$3.0767(-1)$} & & 9 & 15 & $7.3858(-1)$ & $6.4816(1)$ & $3.3694(8)$ \\
\hline & $\begin{array}{l}1.5783 \\
1.5783 \\
1.5783 \\
1.5746 \\
1.5746 \\
1.5731\end{array}$ & $\begin{array}{l}1.8855 \\
1.8833 \\
1.8821 \\
1.8833 \\
1.8821 \\
1.8821\end{array}$ & $\begin{array}{l}2966 \\
2987 \\
2999 \\
2951 \\
2963 \\
2949\end{array}$ & $\begin{array}{l}5 \\
5 \\
5 \\
3 \\
3 \\
1\end{array}$ & $\begin{array}{l}7 \\
5 \\
3 \\
5 \\
3 \\
3\end{array}$ & $\begin{array}{l}6.1946(-1) \\
1.0983(-1) \\
7.2930(-3) \\
5.5579(-1) \\
1.8454(-1) \\
7.4178(-1)\end{array}$ & $\begin{array}{l}3.0247(1) \\
5.4013 \\
3.6009(-1) \\
1.6204(1) \\
5.4013 \\
7.2017\end{array}$ & $\begin{array}{l}3.3540(8) \\
8.2062(7) \\
9.0108(6) \\
2.5526(8) \\
1.4016(8) \\
1.8963(8)\end{array}$ \\
\hline$z^{3} P^{0} \rightarrow a^{3} S^{*}$ & \multicolumn{2}{|c|}{$6.8602(-1)$} & & 9 & 3 & $2.5494(-2)$ & 1.0034 & $2.8912(8)$ \\
\hline & $\begin{array}{l}0.8998 \\
0.9000 \\
0.9001\end{array}$ & $\begin{array}{l}1.5860 \\
1.5860 \\
1.5860\end{array}$ & $\begin{array}{l}1328 \\
1329 \\
1329\end{array}$ & $\begin{array}{l}5 \\
3 \\
1\end{array}$ & $\begin{array}{l}3 \\
3 \\
3\end{array}$ & $\begin{array}{l}2.5499(-2) \\
2.5492(-2) \\
2.5490(-2)\end{array}$ & $\begin{array}{l}5.5742(-1) \\
3.3445(-1) \\
1.1148(-1)\end{array}$ & $\begin{array}{l}1.6071(8) \\
9.6353(7) \\
3.2109(7)\end{array}$ \\
\hline$z^{3} p^{0} \rightarrow b^{3} p^{*}$ & \multicolumn{2}{|c|}{$6.7653(-1)$} & & 9 & 9 & $8.7698(-3)$ & $3.5000(-1)$ & $3.2240(7)$ \\
\hline & $\begin{array}{l}0.8998 \\
0.8998 \\
0.9000 \\
0.9000 \\
0.9000 \\
0.9001\end{array}$ & $\begin{array}{l}1.5783 \\
1.5746 \\
1.5783 \\
1.5746 \\
1.5731 \\
1.5746\end{array}$ & $\begin{array}{l}1343 \\
1351 \\
1344 \\
1351 \\
1354 \\
1351\end{array}$ & $\begin{array}{l}5 \\
5 \\
3 \\
3 \\
3 \\
1\end{array}$ & $\begin{array}{l}5 \\
3 \\
5 \\
3 \\
1 \\
3\end{array}$ & $\begin{array}{l}6.5961(-3) \\
2.1867(-3) \\
3.6636(-3) \\
2.1861(-3) \\
2.9084(-3) \\
8.7438(-3)\end{array}$ & $\begin{array}{l}1.4583(-1) \\
4.8611(-2) \\
4.8611(-2) \\
2.9167(-2) \\
3.8889(-2) \\
3.8889(-2)\end{array}$ & $\begin{array}{l}2.4388(7) \\
1.3328(7) \\
8.1229(6) \\
7.9906(6) \\
3.1750(7) \\
1.0651(7)\end{array}$ \\
\hline$z^{3} P^{0} \rightarrow a^{3} D^{\bullet}$ & \multicolumn{2}{|c|}{$6.5179(-1)$} & & 9 & 15 & $4.3109(-3)$ & $1.7858(-1)$ & $8.8261(6)$ \\
\hline & $\begin{array}{l}0.8998 \\
0.8998 \\
0.8998 \\
0.9000 \\
0.9000 \\
0.9001\end{array}$ & $\begin{array}{l}1.5551 \\
1.5498 \\
1.5471 \\
1.5498 \\
1.5471 \\
1.5471\end{array}$ & $\begin{array}{l}1391 \\
1402 \\
1408 \\
1402 \\
1408 \\
1408\end{array}$ & $\begin{array}{l}5 \\
5 \\
5 \\
3 \\
3 \\
1\end{array}$ & $\begin{array}{l}7 \\
5 \\
3 \\
5 \\
3 \\
3\end{array}$ & $\begin{array}{l}3.6404(-3) \\
6.4482(-4) \\
4.2809(-5) \\
3.2232(-3) \\
1.0699(-3) \\
4.2793(-3)\end{array}$ & $\begin{array}{l}8.3335(-2) \\
1.4881(-2) \\
9.9209(-4) \\
4.4644(-2) \\
1.4881(-2) \\
1.9842(-2)\end{array}$ & $\begin{array}{l}8.9678(6) \\
2.1880(6) \\
2.4009(5) \\
6.5585(6) \\
3.5984(6) \\
4.7965(6)\end{array}$ \\
\hline$y^{3} P^{s} \rightarrow a^{3} S^{4}$ & \multicolumn{2}{|c|}{$2.8175(-1)$} & & 9 & 3 & $7.1667(-3)$ & $6.8677(-1)$ & $1.3709(7)$ \\
\hline & $\begin{array}{l}1.3042 \\
1.3042 \\
1.3040\end{array}$ & $\begin{array}{l}1.5860 \\
1.5860 \\
1.5860\end{array}$ & $\begin{array}{l}3235 \\
3234 \\
3232\end{array}$ & $\begin{array}{l}5 \\
3 \\
1\end{array}$ & $\begin{array}{l}3 \\
3 \\
3\end{array}$ & $\begin{array}{l}7.1679(-3) \\
7.1679(-3) \\
7.1730(-3)\end{array}$ & $\begin{array}{l}3.8154(-1) \\
2.2892(-1) \\
7.6308(-2)\end{array}$ & $\begin{array}{l}7.6201(6) \\
4.5720(6) \\
1.5273(6)\end{array}$ \\
\hline$y^{3} p^{c} \rightarrow b^{3} p^{c}$ & \multicolumn{2}{|c|}{$2.7226(-1)$} & & 9 & 9 & $3.2359(-1)$ & $3.2091(1)$ & $1.9266(8)$ \\
\hline & $\begin{array}{l}1.3042 \\
1.3042 \\
1.3042 \\
1.3042 \\
1.3042 \\
1.3040\end{array}$ & $\begin{array}{l}1.5783 \\
1.5746 \\
1.5783 \\
1.5746 \\
1.5731 \\
1.5746\end{array}$ & $\begin{array}{l}3326 \\
3371 \\
3325 \\
3370 \\
3388 \\
3368\end{array}$ & $\begin{array}{l}5 \\
5 \\
3 \\
3 \\
3 \\
3 \\
1\end{array}$ & $\begin{array}{l}5 \\
3 \\
5 \\
3 \\
1 \\
3\end{array}$ & $\begin{array}{l}2.4434(-1) \\
8.0346(-2) \\
1.3574(-1) \\
8.0346(-2) \\
1.0653(-1) \\
3.2162(-1)\end{array}$ & $\begin{array}{l}1.3371(1) \\
4.4571 \\
4.4571 \\
2.6742 \\
3.5656 \\
3.5656\end{array}$ & $\begin{array}{l}1.4745(8) \\
7.8644(7) \\
4.9150(7) \\
4.7186(7) \\
1.8562(8) \\
6.3055(7)\end{array}$ \\
\hline$y^{3} p^{b} \rightarrow a^{3} D^{c}$ & \multicolumn{2}{|c|}{$2.4752(-1)$} & & 9 & 15 & $2.6247(-1)$ & $2.8631(1)$ & $7.7495(7)$ \\
\hline & $\begin{array}{l}1.3042 \\
1.3042 \\
1.3042 \\
1.3042 \\
1.3042 \\
1.3040\end{array}$ & $\begin{array}{l}1.5551 \\
1.5498 \\
1.5471 \\
1.5498 \\
1.5471 \\
1.5471\end{array}$ & $\begin{array}{l}3613 \\
3711 \\
3753 \\
3710 \\
3752 \\
3749\end{array}$ & $\begin{array}{l}5 \\
5 \\
5 \\
3 \\
3 \\
1\end{array}$ & $\begin{array}{l}7 \\
5 \\
3 \\
5 \\
3 \\
3\end{array}$ & $\begin{array}{l}2.2349(-1) \\
3.9066(-2) \\
2.5757(-3) \\
1.9533(-1) \\
6.4394(-2) \\
2.5779(-1)\end{array}$ & $\begin{array}{l}1.3361(1) \\
2.3859 \\
1.5906(-1) \\
7.1578 \\
2.3859 \\
3.1812\end{array}$ & $\begin{array}{l}8.0717(7) \\
1.8927(7) \\
2.0344(6) \\
5.6782(7) \\
3.0517(7) \\
4.0789(7)\end{array}$ \\
\hline$x^{3} P^{\infty} \rightarrow a^{3} S^{e}$ & \multicolumn{2}{|c|}{$2.4674(-1)$} & & 9 & 3 & $1.6523(-1)$ & $1.8081(1)$ & $2.4241(8)$ \\
\hline & $\begin{array}{l}1.3409 \\
1.3372 \\
1.3368\end{array}$ & $\begin{array}{l}1.5860 \\
1.5860 \\
1.5860\end{array}$ & $\begin{array}{l}3719 \\
3663 \\
3658\end{array}$ & $\begin{array}{l}5 \\
3 \\
1\end{array}$ & $\begin{array}{l}3 \\
3 \\
3\end{array}$ & $\begin{array}{l}1.6413(-1) \\
1.6661(-1) \\
1.6688(-1)\end{array}$ & $\begin{array}{l}1.0045(1) \\
6.0269 \\
2.0090\end{array}$ & $\begin{array}{l}1.3200(8) \\
8.2839(7) \\
2.7747(7)\end{array}$ \\
\hline \multirow[t]{2}{*}{$x^{3} p^{n} \rightarrow b^{3} P^{n}$} & \multicolumn{2}{|c|}{$2.3725(-1)$} & & 9 & 9 & $7.3304(-2)$ & 8.3423 & $3.3141(7)$ \\
\hline & $\begin{array}{l}1.3409 \\
1.3409 \\
1.3372\end{array}$ & $\begin{array}{l}1.5783 \\
1.5746 \\
1.5783\end{array}$ & $\begin{array}{l}3839 \\
3900 \\
3780\end{array}$ & $\begin{array}{l}5 \\
5 \\
3\end{array}$ & $\begin{array}{l}5 \\
3 \\
5\end{array}$ & $\begin{array}{l}5.5013(-2) \\
1.8052(-2) \\
3.1039(-2)\end{array}$ & $\begin{array}{l}3.4760 \\
1.1587 \\
1.1587\end{array}$ & $\begin{array}{l}2.4904(7) \\
1.3199(7) \\
8.6955(6)\end{array}$ \\
\hline
\end{tabular}

Table V(b). (continued) 


\begin{tabular}{|c|c|c|c|c|c|c|c|c|}
\hline \multirow[b]{2}{*}{ Transition } & \multicolumn{8}{|l|}{ S III } \\
\hline & $\begin{array}{l}E_{\mathrm{i}} \\
\text { (Ry) }\end{array}$ & $\begin{array}{l}E_{\mathrm{f}} \\
\text { (Ry) }\end{array}$ & $\begin{array}{l}W L \\
(\AA)\end{array}$ & $g_{i}$ & $g_{f}$ & $f_{\text {fit }}$ & $s$ & $\begin{array}{l}a_{33} \\
\left(\mathrm{sec}^{-1}\right)\end{array}$ \\
\hline & 1.3372 & 1.5746 & 3839 & 3 & 3 & $1.8338(-2)$ & $6.9520(-1)$ & $8.3012(6)$ \\
\hline & 1.3372 & 1.5731 & 3862 & 3 & 1 & $2.4296(-2)$ & $9.2693(-1)$ & $3.2580(7)$ \\
\hline & 1.3368 & 1.5746 & 3833 & 1 & 3 & $7.3474(-2)$ & $9.2693(-1)$ & $1.1142(7)$ \\
\hline \multirow[t]{7}{*}{$x^{3} p^{\circ} \rightarrow a^{3} D^{0}$} & \multicolumn{2}{|c|}{$2.1251(-1)$} & & 9 & 15 & $2.7295(-1)$ & $3.4679(1)$ & $5.9405(7)$ \\
\hline & 1.3409 & 1.5551 & 4255 & 5 & 7 & $2.3110(-1)$ & $1.6183(1)$ & $6.0834(7)$ \\
\hline & 1.3409 & 1.5498 & 4363 & $s$ & 5 & $4.0247(-2)$ & 2.8899 & $1.4107(7)$ \\
\hline & 1.3409 & 1.5471 & 4420 & 5 & 3 & $2.6484(-3)$ & $2.9266(-1)$ & $1.5075(6)$ \\
\hline & 1.3372 & 1.5498 & 4286 & 3 & 5 & $2.0480(-1)$ & 8.6697 & $4.4611(7)$ \\
\hline & 1.3372 & 1.5471 & 4341 & 3 & 3 & $6.7399(-2)$ & 2.8899 & $2.3851(7)$ \\
\hline & 1.3368 & 1.5471 & 4334 & 1 & 3 & $27011(-1)$ & 3.8532 & $3.1984(7)$ \\
\hline \multirow[t]{7}{*}{$a^{3} D^{*} \rightarrow w^{3} p^{\infty}$} & \multicolumn{2}{|c|}{$3.4371(-1)$} & & 15 & 9 & $8.2838(-4)$ & $1.0845(-1)$ & $1.3101(6)$ \\
\hline & 1.5551 & 1.8945 & 2685 & 7 & 5 & $8.1799(-4)$ & $5.0612(-2)$ & $1.0596(6)$ \\
\hline & 1.5498 & 1.8945 & 2644 & 5 & 5 & $2.0769(-4)$ & $9.0379(-3)$ & $1.9821(5)$ \\
\hline & 1.5471 & 1.8945 & 2623 & 3 & 5 & $2.3257(-5)$ & $6.0252(-4)$ & $1.3527(4)$ \\
\hline & 1.5498 & 1.8964 & 2629 & 5 & 3 & $6.2650(-4)$ & $2.7114(-2)$ & $1.0075(6)$ \\
\hline & 1.5471 & 1.8964 & 2609 & 3 & 3 & $3.5077(-4)$ & $9.0379(-3)$ & 3.4376 (5) \\
\hline & 1.5471 & 1.8974 & 2601 & 3 & 1 & $4.6903(-4)$ & $1.2050(-2)$ & $1.3869(6)$ \\
\hline \multirow[t]{7}{*}{$a^{3} D^{0} \rightarrow v^{3} P^{a}$} & \multicolumn{2}{|c|}{$3.6504(-1)$} & & 15 & 9 & $1.8411(-1)$ & $2.2695(1)$ & $3.2842(8)$ \\
\hline & 1.5551 & 1.9200 & 2497 & 7 & 5 & $1.8403(-1)$ & $1.0591(1)$ & $2.7556(8)$ \\
\hline & 1.5498 & 1.9200 & 2461 & 5 & 5 & $4.6677(-2)$ & 1.8913 & $5.1382(7)$ \\
\hline & 1.5471 & 1.9200 & 2443 & 3 & 5 & $5.2241(-3)$ & $1.2609(-1)$ & $3.5010(6)$ \\
\hline & 1.5498 & 1.9130 & 2509 & 5 & 3 & $1.3738(-1)$ & 5.6738 & $2.4261(8)$ \\
\hline & 1.5471 & 1.9130 & 2490 & 3 & 3 & $7.6891(-2)$ & 1.8913 & $8.2687(7)$ \\
\hline & 1.5471 & 1.9116 & 2500 & 3 & 1 & $1.0213(-1)$ & 2.5217 & $3.2697(8)$ \\
\hline \multirow[t]{8}{*}{$a^{3} D^{*} \rightarrow x^{3} D^{a}$} & \multicolumn{2}{|c|}{$3.3241(-1)$} & & 15 & 15 & $1.5730(-1)$ & $2.1295(1)$ & $1.3961(8)$ \\
\hline & 1.5551 & 1.8855 & 2758 & 7 & 7 & $1.3893(-1)$ & 8.8304 & $1.2182(8)$ \\
\hline & 1.5551 & 1.8833 & 2776 & 7 & 5 & $1.7306(-2)$ & 1.1073 & $2.0963(7)$ \\
\hline & 1.5498 & 1.8855 & 2714 & 5 & 7 & $2.4782(-2)$ & 1.1073 & $1.6023(7)$ \\
\hline & 1.5498 & 1.8833 & 2732 & 5 & 5 & $1.0984(-1)$ & 4.9405 & $9.8130(7)$ \\
\hline & 1.5498 & 1.8821 & 2742 & 5 & 3 & $2.3588(-2)$ & 1.0648 & $3.4869(7)$ \\
\hline & 1.5471 & 1.8833 & 2710 & 3 & 5 & $3.9775(-2)$ & 1.0648 & $2.1667(7)$ \\
\hline & 2.5471 & 1.8821 & 2720 & 3 & 3 & $1.1890(-1)$ & 3.1943 & $1.0718(8)$ \\
\hline \multirow[t]{7}{*}{$a^{3} D^{2} \rightarrow y^{3} F^{0}$} & \multicolumn{2}{|c|}{$3.1789(-1)$} & & 15 & 21 & $9,0781(-1)$ & $1.285 !(2)$ & $5.2634(8)$ \\
\hline & 1.5551 & 1.8732 & 2864 & 7 & 9 & $8.3425(-1)$ & $5.5075(1)$ & $5.2737(8)$ \\
\hline & 1.5551 & 1.8688 & 2905 & 7 & 7 & $7.1301(-2)$ & 4.7731 & $5.6359(7)$ \\
\hline & 1.5551 & 1.8643 & 2947 & 7 & 5 & $1.9822(-3)$ & $1.3463(-1)$ & $2.1311(6)$ \\
\hline & 1.5498 & 1.8688 & 2857 & 5 & 7 & $8.0947(-1)$ & 3.8063 (1) & $4.7259(8)$ \\
\hline & 1.5498 & 1.8643 & 2898 & 5 & 5 & $1.0008(-1)$ & 4.7731 & 7.9508 (7) \\
\hline & 1.5471 & 1.8643 & 2873 & 3 & 5 & $9.0583(-1)$ & $2.5701(1)$ & $4.3924(8)$ \\
\hline \multirow[t]{7}{*}{$z^{3} D^{6} \rightarrow b^{3} p^{4}$} & \multicolumn{2}{|c|}{$8.1038(-1)$} & & 15 & 9 & $1.8926(-2)$ & 1.0510 & $1.6639(8)$ \\
\hline & 0.7664 & 1.5783 & 1122 & 7 & 5 & $1.8962(-2)$ & $4.9045(-1)$ & $1.4057(8)$ \\
\hline & 0.7659 & 1.5783 & 1122 & 5 & 5 & $4.7433(-3)$ & $8.7580(-2)$ & $2.5145(7)$ \\
\hline & 0.7656 & 1.5783 & $\$ 121$ & 3 & 5 & $5.2721(-4)$ & $5.8387(-3)$ & $1.6780(6)$ \\
\hline & 0.7659 & 1.5746 & 1127 & 5 & 3 & $1.4165(-2)$ & $2.6274(-1)$ & $1.2402(8)$ \\
\hline & 0.7656 & 1.5746 & 1127 & 3 & 3 & $7.8721(-3)$ & $8.7580(-2)$ & $4.1379(7)$ \\
\hline & 0.7656 & 1.5731 & 1129 & 3 & 1 & $1.0477(-2)$ & $1.1677(-1)$ & $1.6460(8)$ \\
\hline \multirow[t]{8}{*}{$z^{3} D^{\circ} \rightarrow a^{3} D^{\circ}$} & & & & 15 & 15 & $8.2806(-3)$ & $4.7430(-1)$ & $4.1054(7)$ \\
\hline & 0.7664 & 1.5551 & 1155 & 7 & 7 & $7.3868(-3)$ & $1.9667(-1)$ & $3.6911(7)$ \\
\hline & 0.7664 & 1.5498 & 1163 & 7 & 5 & $9.2010(-4)$ & $2.4663(-2)$ & $6.3504(6)$ \\
\hline & 0.7659 & 1.5551 & 1155 & 5 & 7 & $1.2976(-3)$ & $2.4663(-2)$ & $4.6369(6)$ \\
\hline & 0.7659 & 1.5498 & 1162 & 5 & 5 & $5.7505(-3)$ & $1.1004(-1)$ & $2.8383(7)$ \\
\hline & 0.7659 & 1.5471 & 1167 & 5 & 3 & $1.2351(-3)$ & $2.3715(-2)$ & $1.0090(7)$ \\
\hline & 0.7656 & 1.5498 & 1162 & 3 & 5 & $2.0662(-3)$ & $2.3715(-2)$ & $6.1232(6)$ \\
\hline & 0.7656 & 1.5471 & 1166 & 3 & 3 & $6.1774(-3)$ & $7.1144(-2)$ & $3.0301(7)$ \\
\hline$y^{3} D^{0} \rightarrow b^{3} P^{4}$ & & & & 15 & 9 & $7,2797(-2)$ & $1.4205(1)$ & $5.1827(7)$ \\
\hline & 1.3464 & 1.5783 & 3930 & 7 & 5 & $7.3205(-2)$ & 6.6291 & $4.4269(7)$ \\
\hline & 1.3459 & 1.5783 & 3921 & 5 & 5 & $1.8341(-2)$ & 1.1838 & 7.9565 (6) \\
\hline & 1.3446 & 1.5783 & 3900 & 3 & 5 & $2.0492(-3)$ & $7.8918(-2)$ & 5.3938 (5) \\
\hline
\end{tabular}

Table V(b). (continued) 


\begin{tabular}{|c|c|c|c|c|c|c|c|c|}
\hline \multirow[b]{2}{*}{ Transition } & \multicolumn{8}{|l|}{ S III } \\
\hline & $\begin{array}{l}E_{\mathrm{i}} \\
\text { (Ry) }\end{array}$ & $\begin{array}{l}E_{\mathrm{f}} \\
\text { (Ry) }\end{array}$ & $\begin{array}{l}W L \\
(\mathrm{~A})\end{array}$ & $g_{\mathrm{i}}$ & $\theta_{r}$ & $f_{\mathrm{H}}$ & $s$ & $\begin{array}{l}a_{j 1} \\
\left(\sec ^{-1}\right)\end{array}$ \\
\hline & 1.3459 & 1.5746 & 3985 & 5 & 3 & $5.4146(-2)$ & 3.5513 & $3.7912(7)$ \\
\hline & 1.3446 & 1.5746 & 3963 & 3 & 3 & $3.0252(-2)$ & 1.1838 & $1.2854(7)$ \\
\hline & 1.3446 & 1.5731 & 3987 & 3 & 1 & $4.0073(-2)$ & 1.5784 & $5.0417(7)$ \\
\hline \multirow[t]{8}{*}{$y^{3} D^{\circ} \rightarrow a^{3} D^{4}$} & \multicolumn{2}{|c|}{$2.0587(-1)$} & & 15 & 15 & $2.5007(-2)$ & 5.4660 & $8.5129(6)$ \\
\hline & 1.3464 & 1.5551 & 4366 & 7 & 7 & $2.2525(-3)$ & 2.2666 & $7.8805(6)$ \\
\hline & 1.3464 & 1.5498 & 4480 & 7 & 5 & $2.7530(-3)$ & $2.8423(-1)$ & $1.2808(6)$ \\
\hline & 1.3459 & 1.5551 & 4356 & 5 & 7 & $3.9641(-3)$ & $2.8423(-1)$ & $9.9535(5)$ \\
\hline & 1.3459 & 1.5498 & 4469 & 5 & 5 & $1.7238(-2)$ & 1.2681 & $5.7565(6)$ \\
\hline & 1.3459 & 1.5471 & 4529 & 5 & 3 & $3.6659(-3)$ & $2.7330(-1)$ & $1.9866(6)$ \\
\hline & 1.3446 & 1.5498 & 4441 & 3 & 5 & $6.2313(-3)$ & $2.7330(-1)$ & $1.2645(6)$ \\
\hline & 1.3446 & 1.5471 & 4500 & 3 & 3 & $1.8448(-2)$ & $8.1990(-1)$ & $6.0762(6)$ \\
\hline \multirow[t]{7}{*}{$z^{3} F^{\circ} \rightarrow a^{3} D^{e}$} & \multicolumn{2}{|c|}{$4.3536(-1)$} & & 21 & 15 & $1.4146(-1)$ & $2.0470(1)$ & $3.0151(8)$ \\
\hline & 1.1190 & 1.5551 & 2090 & 9 & 7 & $1.4170(-1)$ & 8.7728 & $2.7830(8)$ \\
\hline & 1.1154 & 1.5551 & 2073 & 7 & 7 & $1.5919(-2)$ & $7.6031(-1)$ & $2.4722(7)$ \\
\hline & 1.1128 & 1.5551 & 2060 & 5 & 7 & $6.3233(-4)$ & $2.1445(-2)$ & $7.0972(5)$ \\
\hline & 1.1154 & 1.5498 & 2098 & 7 & 5 & $1.2542(-1)$ & 6.0630 & $2.6614(8)$ \\
\hline & 1.1128 & 1.5498 & 2085 & 5 & 5 & $2.2150(-2)$ & $7.6031(-1)$ & $3.3977(7)$ \\
\hline & 1.1128 & 1.5471 & 2099 & 5 & 3 & $1.1853(-1)$ & 4.0940 & $2.9930(8)$ \\
\hline
\end{tabular}

Table V(c). $f$ - and A-values for Ar V 


\begin{tabular}{|c|c|c|c|c|c|c|c|c|}
\hline \multirow[b]{2}{*}{ Transition } & \multicolumn{8}{|l|}{ Ar V } \\
\hline & $\begin{array}{l}E_{\mathrm{j}} \\
\text { (Ry) }\end{array}$ & $\begin{array}{l}E_{\mathrm{r}} \\
\text { (Ry) }\end{array}$ & $\begin{array}{l}W L \\
(\mathcal{A})\end{array}$ & $g_{1}$ & $g_{1}$ & $f_{u}$ & $s$ & $\begin{array}{l}a_{j} \\
\left(\sec ^{-1}\right)\end{array}$ \\
\hline$a^{1} S^{4} \rightarrow z^{1} P^{0}$ & 0.3455 & 1.7802 & 635 & 1 & 3 & $1.1346(-1)$ & $2.3724(-1)$ & $6.2528(8)$ \\
\hline$a^{1} S^{e} \rightarrow y^{1} p^{0}$ & 0.3455 & 2.2976 & 467 & 1 & 3 & 2.2398 & 3.4422 & $2.2853(10)$ \\
\hline$a^{1} S^{0} \rightarrow x^{1} P^{0}$ & 0.3455 & 2.7456 & 380 & 1 & 3 & $1.5454(-1)$ & $1.9316(-1)$ & 2.3835 (9) \\
\hline$a^{1} D^{4} \rightarrow z^{1} P^{0}$ & 0.1485 & 1.7802 & 558 & 5 & 3 & $2.7960(-1)$ & 2.5704 & 9.9653 (9) \\
\hline$a^{1} D^{*} \rightarrow y^{1} P^{\circ}$ & 0.1485 & 2.2976 & 424 & 5 & 3 & $3.3710(-3)$ & $2.3529(-2)$ & $2.0842(8)$ \\
\hline$a^{1} D^{e} \rightarrow x^{1} P^{\circ}$ & 0.1485 & 2.7456 & 351 & 5 & 3 & $1.4288(-1)$ & $8.2522(-1)$ & $1.2901(10)$ \\
\hline$a^{1} D^{\varphi} \rightarrow z^{1} D^{\circ}$ & 0.1485 & 1.4053 & 725 & 5 & 5 & $6.5355(-2)$ & $7.8003(-1)$ & $8.2914(8)$ \\
\hline$a^{2} D^{\cdot} \rightarrow z^{2} F^{\circ}$ & 0.1485 & 2.2354 & 437 & 5 & 7 & 1.1903 & 8.5557 & $2.9741(10)$ \\
\hline \multirow[t]{4}{*}{$a^{3} p^{t} \rightarrow z^{3} S^{0}$} & \multicolumn{2}{|c|}{1.7328} & & 9 & 3 & $2.9778(-1)$ & 4.6399 & $2.1546(10)$ \\
\hline & 0.0185 & 1.7454 & 528 & 5 & 3 & $2.9676(-1)$ & 2.5777 & $1.1848(10)$ \\
\hline & 0.0070 & 1.7454 & 524 & 3 & 3 & $2.9874(-1)$ & 1.5466 & $7.2520(9)$ \\
\hline & 0.0000 & 1.7454 & 522 & 1 & 3 & $2.9994(-1)$ & $5.1554(-1)$ & $2.4465(9)$ \\
\hline \multirow[t]{7}{*}{$a^{3} P^{0} \rightarrow z^{3} P^{0}$} & \multicolumn{2}{|c|}{1.2793} & & 9 & 9 & $6.0916(-2)$ & 1.2857 & $8.0077(8)$ \\
\hline & 0.0185 & 1.2918 & 716 & 5 & 5 & $4.5473(-2)$ & $5.3569(-1)$ & 5.9219 (8) \\
\hline & 0.0185 & 1.2919 & 716 & 5 & 3 & $1.5159(-2)$ & $1.7856(-1)$ & $3.2907(8)$ \\
\hline & 0.0070 & 1.2918 & 709 & 3 & 5 & $2.5492(-2)$ & $1.7856(-1)$ & $2.0281(8)$ \\
\hline & 0.0070 & 1.2919 & 709 & 3 & 3 & $1.5296(-2)$ & $1.0714(-1)$ & $2.0285(8)$ \\
\hline & 0.0070 & 1.2919 & 709 & 3 & 1 & $2.0395(-2)$ & $1.4285(-1)$ & $8.1142(8)$ \\
\hline & 0.0000 & 1.2919 & 705 & 1 & 3 & $6.1516(-2)$ & $1.4285(-1)$ & $2.7489(8)$ \\
\hline \multirow[t]{7}{*}{$a^{3} P^{4} \rightarrow y^{3} P^{0}$} & \multicolumn{2}{|c|}{1.9734} & & 9 & 9 & $5.7123(-1)$ & 7.8157 & $1.7867(10)$ \\
\hline & 0.0185 & 1.9827 & 464 & 5 & 5 & $4.2643(-1)$ & 3.2565 & $1.3215(10)$ \\
\hline & 0.0185 & 1.9892 & 462 & 5 & 3 & $1.4262(-1)$ & 1.0855 & 7.4147 (9) \\
\hline & 0.0070 & 1.9827 & 461 & 3 & 5 & $2.3830(-1)$ & 1.0855 & $4.4830(9)$ \\
\hline & 0.0070 & 1.9892 & 460 & 3 & 3 & $1.4345(-1)$ & $6.5131(-1)$ & $4.5274(9)$ \\
\hline & 0.0070 & 1.9924 & 459 & 3 & 1 & $1.9157(-1)$ & $8.6841(-1)$ & $1.8197(10)$ \\
\hline & 0.0000 & 1.9892 & 458 & 1 & 3 & $5.7581(-1)$ & $8.6841(-1)$ & $6.1003(9)$ \\
\hline \multirow[t]{7}{*}{$a^{3} p^{c} \rightarrow x^{3} p^{\circ}$} & \multicolumn{2}{|c|}{2.6948} & & 9 & 9 & $1.4379(-1)$ & 1.4406 & $8.3872(9)$ \\
\hline & 0.0185 & 2.7146 & 338 & 5 & 5 & $1.0789(-1)$ & $6.0025(-1)$ & $6.2993(9)$ \\
\hline & 0.0185 & 2.6996 & 340 & 5 & 3 & $3.5763(-2)$ & $2.0008(-1)$ & $3.4415(9)$ \\
\hline & 0.0070 & 2.7146 & 337 & 3 & 5 & $6.0195(-2)$ & $2.0008(-1)$ & 2.1268 (9) \\
\hline & 0.0070 & 2.6996 & 338 & 3 & 3 & $3.5917(-2)$ & $1.2005(-1)$ & $2.0917(9)$ \\
\hline & 0.0070 & 2.6950 & 339 & 3 & 1 & $4.7808(-2)$ & $1.6007(-1)$ & $8.3239(9)$ \\
\hline & 0.0000 & 2.6996 & 338 & 1 & 3 & $1.4404(-1)$ & $1.6007(-1)$ & $2.8106(9)$ \\
\hline \multirow[t]{7}{*}{$a^{3} P^{*} \rightarrow z^{3} D^{a}$} & \multicolumn{2}{|c|}{1.0967} & & 9 & 15 & $4.2832(-2)$ & 1.0545 & $2.4827(8)$ \\
\hline & 0.0185 & 1.1100 & 835 & 5 & 7 & $3.5808(-2)$ & $4.9210(-1)$ & $2.4475(8)$ \\
\hline & 0.0185 & 1.1088 & 836 & 5 & 5 & $6.3873(-3)$ & $8.7874(-2)$ & $6.0990(7)$ \\
\hline & 0.0185 & 1.1084 & 836 & 5 & 3 & $4.2567(-4)$ & $5.8583(-3)$ & $6.7692(6)$ \\
\hline & 0.0070 & 1.1088 & 827 & 3 & 5 & $3.2274(-2)$ & $2.6362(-1)$ & $1.8884(8)$ \\
\hline & 0.0070 & 1.3084 & 827 & 3 & 3 & $1.0754(-2)$ & $8.7874(-2)$ & $1.0479(8)$ \\
\hline & 0.0000 & 1.1084 & 822 & 1 & 3 & $4.3289(-2)$ & $1.1717(-1)$ & $1.4239(8)$ \\
\hline \multirow[t]{7}{*}{$a^{3} P^{e} \rightarrow y^{3} D^{\circ}$} & \multicolumn{2}{|c|}{2.0336} & & 9 & 15 & 1.3865 & $1.8408(1)$ & $27634(10)$ \\
\hline & 0.0185 & 2.0478 & 449 & 5 & 7 & 1.1622 & 8.5905 & $2.7459(10)$ \\
\hline & 0.0185 & 2.0458 & 449 & 5 & 5 & $2.0733(-1)$ & 1.5340 & $6.8444(9)$ \\
\hline & 0.0185 & 2.0432 & 450 & 5 & 3 & $1.3804(-2)$ & $1.0227(-1)$ & $7.5757(8)$ \\
\hline & 0.0070 & 20458 & 447 & 3 & 5 & 1.0425 & 4.6021 & $2.0886(10)$ \\
\hline & 0.0070 & 2.0432 & 448 & 3 & 3 & $3.4707(-1)$ & 1.5340 & $1.1559(10)$ \\
\hline & 0.0000 & 2.0432 & 446 & 1 & 3 & 1.3930 & 2.0454 & $1.5570(10)$ \\
\hline
\end{tabular}

Table V(d). $f$ - and A-values for Ca VII 


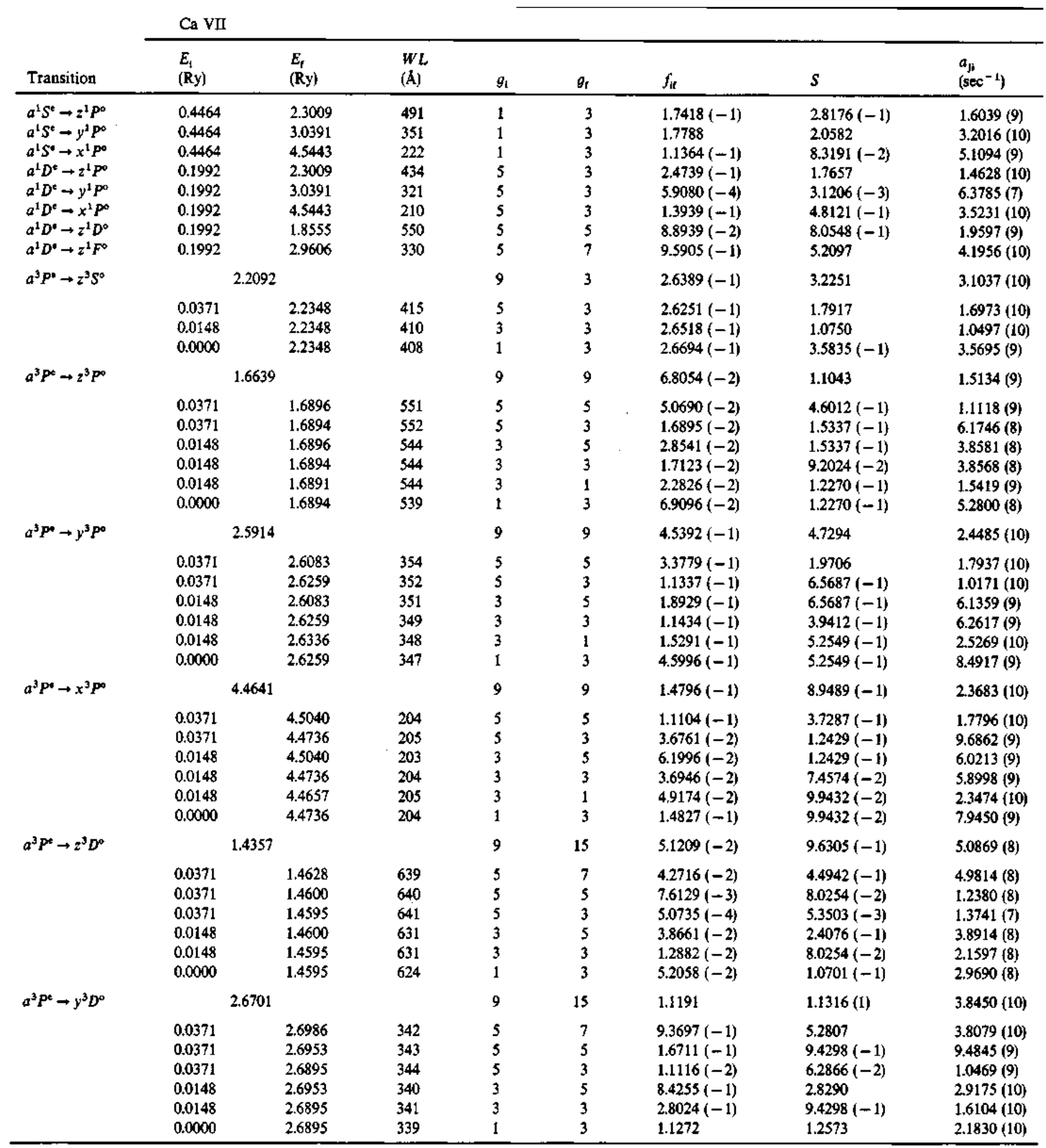

\section{References}


1. Seaton, M. J., J. Phys. B20, 6363 (1987); Berrington, K. A., Burke, P. G., Butler, K., Seaton, M. J., Storey, P. J., Taylor, K. T. and Yu, Y., J. Phys. B20, 6379 (1987).

2. Nahar, S. N. and Pradhan, A. K., J. Phys. B26, 1109 (1993).

3. Allen, C. W., "Astrophysical Quantities" (3rd Edition) (Athlone Press, London 1976).

4. O'Brian, T. R. and Lawler, J. E., Phys. Rev. A44, 7134 (1991).

5. Becker U., Kerkhoff, H., Kwiatkowski, M., Schmidt, M., Teppner, U. and Zimmermann, P., Phys. Lett. 76A, 125 (1980).

6. Berry, H. G., Schectman, R. M., Martinsen, I., Bickel, W. S. and Bashkin, S. J., J. Opt. Soc. Am. 60, 335 (1970).

7. Livingston, A. E., Dumont, P. D., Baudinet-Robinet, Y., Garnir, H. P., Biemont, E. and Grevesse, N., in: "Beam-Foil Spectroscopy" (Edited by I. A. Sellin and D. J. Pegg) (Plenum, New York 1976), p. 339.

8. Irwin, D. J. G., Livingston, A. E. and Kernahan, J. A., Nucl. Inst. Meth. 110, 111(1973).

9. Ryan, L. J., Rayburn, L. A. and Cunningham, A. J., J. Quant. Spectro-sc. Radiat. Transfer 42, 295 (1989).

10. Martin, W. C. and Zalubas, R., J. Phys. Chem. Ref. Data 12, 323 (1983); Moore, C. E., "Atomic Energy Levels", Natl. Bur. Stand. (U.S.) Circ. No. 467 (U.S. GPO Washington, D.C. 1949); Johansson, L., Magnusson, C. E., Joelsson, I., Zetterberg, P. O., Physica Scripta (1992, in press), their relative energies have been scaled with respect to the ionization energy; Martin, W. C, Zalubus, R., Musgrove, A., J.

Phys. Chem. Ref. Data 19, 821 (1990); Kelly, R. L., unpublished compilation, closely corresponds to: Kelly, R. L., J. Phys. Chem. Ref. Data 16, Suppl. 2 (1987) (private communication, NIST); Sugar, J. and Corliss, C, J. Phys. Chem. Ref. Data 14, Suppl. 2 (1985).

11. Mendoza, C. and Zeippen, C. J., J. Phys. B21, 259 (1988).

12. Ho, Y. K. and Henry, R. J, Physica Scripta 35, 831 (1987).

13. Biemont, E., J. Opt. Soc. Am. B3, 163 (1986).

14. Bergstrom, H., Fans, G. W., Hallstadius, H., Lundberg, H. and Persson, A., Z. Phys. D13, 29 (1989).

15. Dumont, P. D., Garnir, H. P. and Baudinet-Robinet, Y., J. Opt. Soc. Am. 68, 825 (1978).

16. Irwin, D. J. G. and Livingston, A. E., Can. J. Phys. 54, 805 (1976).

17. Livingston, A. E., Pinnington, E. H., Irwin, D. J. G., Kernahan, J. A. and Brooks, R. L., J. Opt. Soc. Am. 71, 442 (1981). 